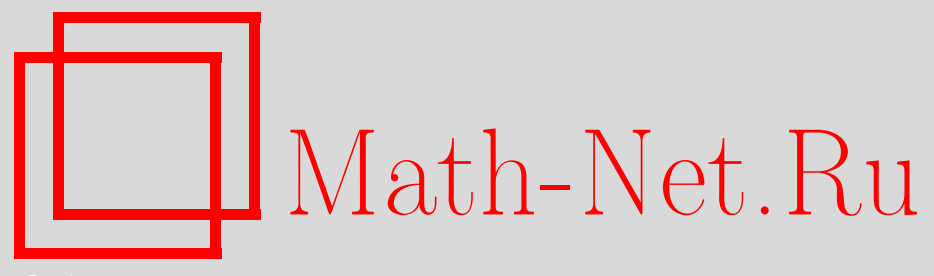

С. А. Назаров, Неравенства Корна для упругих сочленений массивных тел, тонких пластин и стержней, $У М H$, 2008, том 63, выпуск 1, 37-110

DOI: https://doi.org/10.4213/rm8545

Использование Общероссийского математического портала Math-Net.Ru подразумевает, что вы прочитали и согласны с пользовательским соглашением http://www . mathnet.ru/rus/agreement

Параметры загрузки:

IP : 54.224 .60 .19

26 апреля 2023 г., 17:01:41

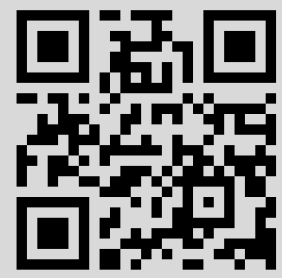




\title{
Неравенства Корна для упругих сочленений массивных тел, тонких пластин и стержней
}

\author{
С. А. Назаров
}

\begin{abstract}
Для сочленений массивных упругих тел и тонких пластин и стержней во всевозможных их комбинациях - получены неравенства Корна, асимптотическая точность которых достигается путем введения разнообразных весовых множителей в $L_{2}$-нормы смещений и их производных. Поскольку тонкие тела по-разному реагируют на растяжение и изгиб, такие неравенства Корна по необходимости становятся анизотропными. Допускаются сочленения упругих тел с контрастными жесткостями, но постоянные в установленных неравенствах не зависят от обоих параметров: относительной толщины $h \in(0,1]$ и относительной жесткости $\mu \in(0,+\infty)$. Нормы, отвечающие жестко защемленным элементам конструкции, существенно отличаются от норм, отвечающих малоподвижным или подвижным элементам, которые не закреплены непосредственно, но только при помощи соседних элементов, - поэтому адекватная структура весовых анизотропных норм определяется геометрией всего сочленения. Каждый вариант неравенства Корна сопровождается примером, подтверждающим оптимальность подбора весовых множителей.
\end{abstract}

Библиография: 77 названий.

\section{СОДЕРЖАНИЕ}

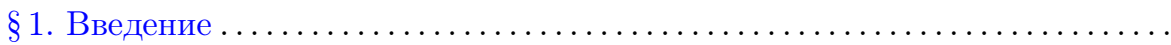

1.1. Сочленения упругих тел с различными предельными размер-

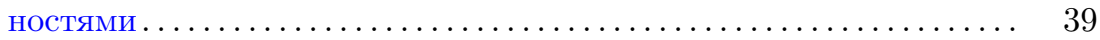

1.2. Весовые анизотропные неравенства Корна ................. 39

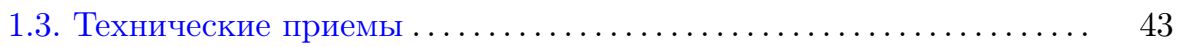

1.4. Структура статьи.................................. 44

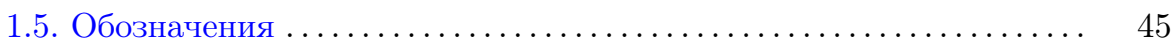

Работа выполнена при финансовой поддержке the Netherlands Organization for Scientific Research (NWO) и Российского фонда фундаментальных исследований (РФФИ); проекты 047.017.020 и 03-01-00835.

(C) C. А. НАзаров, 2008 
$\S 2$ Неравенства Корна для одиночных тел..................... 46

2.1. Массивное тело................................ 46

2.2. Массивное тело, закрепленное по малым множествам ........ 50

2.3. Шпаклевка каверн и шлифовка поверхности ............... 54

2.4. Неравенство Корна для тонкой пластины ............... 56

2.5. Пластины с малыми дефектами или зонами защемления ....... 59

2.6. Неравенство Корна для тонкого стержня................. 60

2.7. Составные тела с контрастными жесткостями............. 63

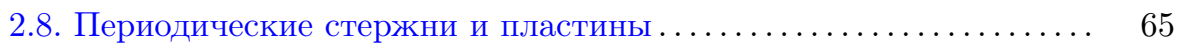

$\S 3$. Неравенства Корна для сочленения массивного тела со стержнями... 66

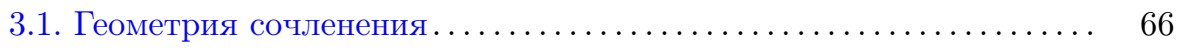

3.2. Специальное расположение шести или более стержней....... 67

3.3. Произвольное расположение стержней................. 71

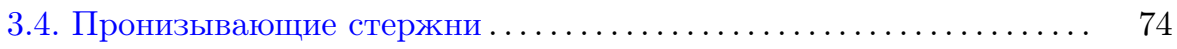

3.5. Незащемленные стержни. ....................... 75

$\S 4$. Неравенства Корна для сочленения массивного тела с пластинами ... 76

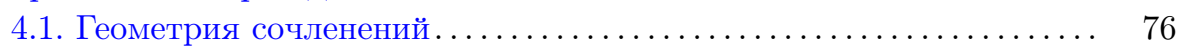

4.2. Пластины, вставленные в неглубокие пазы . . . . . . . . . . . . 77

4.3. Пластины, вставленные в глубокие прорези ............... 79

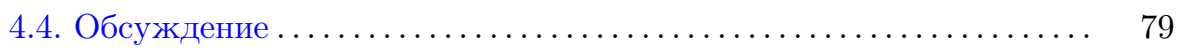

4.5. Незакрепленные пластины . . . . . . . . . . . . . . . . 82

$\S 5$. Неравенства Корна для сочленения пластины со стержнями........ 83

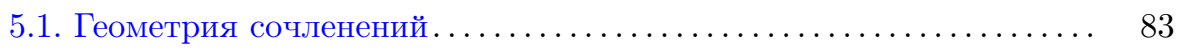

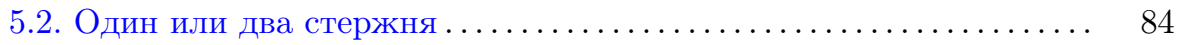

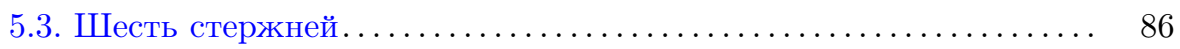

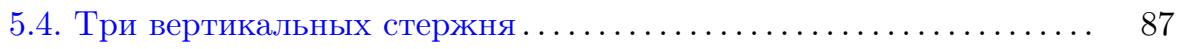

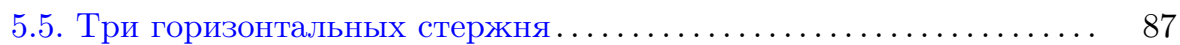

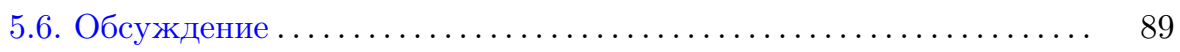

5.7. Стержни, пронизывающие пластину в продольном направлении 90

5.8. Стержень, присоединенный к закрепленной пластине ......... 91

$\S 6$. Неравенства Корна для сочленения тонких пластин . . . . . . . . . . 92

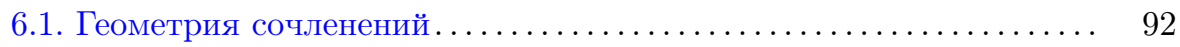

6.2. Неравенства Корна без ограничений на строение сочленения .. . 94

6.3. Неравенство Корна при специальном строении сочленения .... 95

6.4. Обсуждение ....................................... 95

6.5. О передаче весового неравенства неравенства Корна с одной пла-

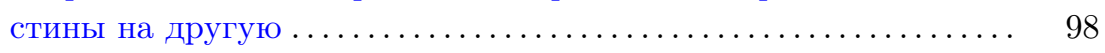

$\S 7$. Неравенства Корна для сочленения тонких стержней ............ 98

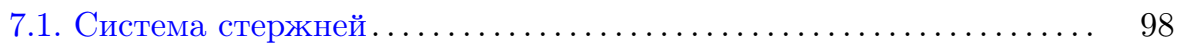

7.2. Защемленные узлы и стержни . . . . . . . . . . . . . . . . 100

7.3. Малоподвижные узлы . . . . . . . . . . . . . . . . . . . . . . 100

7.4. Малоподвижные стержни . . . . . . . . . . . . . . . . . . . . 101

7.5. Подвижные узлы и балки . . . . . . . . . . . . . . . . . . 102

7.6. Неравенство Корна для системы стержней ................. 102

7.7. Обсуждение ................................... 104

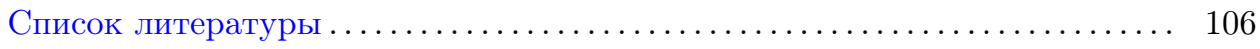




\section{$\S 1$. Введение}

\section{1. Сочленения упругих тел с различными предельными размер-} ностями. Практически в любой инженерной конструкции удается обнаружить фрагменты, состоящие из нескольких или большого числа тонких элементов, которые соединены или закреплены различными способами, но работают как единое целое. В математике такие конгломераты принято называть сочленениями или мультиструктурами. В прикладных дисциплинах, имеющих дело с сопротивлением материалов, известны приближенные методы, позволяющие делать выводы о жесткости так называемых статически определимых стержневых систем, однако многие, встречающиеся на каждом шагу сочленения стержней (оконные переплеты, перила, усложненные фермы и пр.), не поддаются анализу при помощи упомянутых методов, так как включают noдвижные элементы (подробности см. в §7). Для других типов мультиструктур, например таких, как стержни/пластины, сколь-нибудь обоснованных или общепринятых методов предсказания жесткостных свойств нет.

Большинство публикаций, относящихся к математическим исследованиям упругих сочленений, также содержат разнообразные геометрические условия, высказанные явно или неявно - в виде постулируемой априорной оценки решений сингулярно возмущенной задачи теории упругости.

Настоящий обзор посвящен одному из аспектов асимптотического анализа упругих мультиструктур, а именно, неравенствам Корна, не только предоставляющим априорные энергетические оценки решений, но позволяющим определять порядки тех или иных функционалов и предсказывать строение асимптотических анзацев для решений, а также собственных чисел и собственных вектор-функций. В обзоре собраны результаты, полученные автором на протяжении долгого промежутка времени и поэтому нуждавшиеся в унификации, частичной переработке и дополнениях. В итоге удалось создать единый подход к изучению конструкций, которые образованы - во всевозможных их комбинациях - массивными трехмерными телами и тонкими пластинами и стержнями, обладающими "предельными" размерностями 3, 2 и 1 соответственно. Допускаются структуры с контрастными упругими жесткостями, т. е. в дополнение к малому параметру $h \in(0,1]$ - относительной толщине упругих тел вводится параметр $\mu>0$, характеризующий их относительную жесткость.

Если часть поверхности элемента конструкции жестко защемлена, то для него неравенство Корна получается без какого-либо конфигурационного анализа и обычно оказывается асимптотически точным. Поэтому основное внимание уделяется сочленениям, у которых имеются фрагменты, закрепленные лишь посредством соседних элементов (см. приведенные далее многочисленные рисунки).

1.2. Весовые анизотропные неравенства Корна. Известные автору способы доказательства неравенства Корна и его вариантов (см. [1]-[7] и др.) не позволяют выявить зависимость постоянной Корна $C(\Xi)$ от области $\Xi \subset \mathbb{R}^{3}$. Исключение составляет один из результатов В. А. Кондратьева и О. А. Олейник [7] (см. далее теорему 2.2), в котором неравенство Корна распространяется 
с шара на объемлющую область, а постоянный множитель в мажоранте выражается через отношение диаметров. Однако непосредственное применение этого результата к одиночным тонким телам или сочленениям не приводит к успеху, так как при $h \rightarrow+0$ их диаметр остается равным $O(1)$. Ситуация усугубляется тем, что пластины и стержни по-разному реагируют на изгиб и растяжение - подобные анизотропные свойства находят отражение в асимптотических анзацах для упругих полей, а значит, должны быть учтены в соответствующих неравенствах Корна. Таким образом, для многих мультиструктур выяснение зависимости постоянной $C(\Xi)$ от геометрических параметров может оказаться бесполезным.

Изменим саму постановку вопроса, а именно, зафиксируем мажоранту $c \mathscr{E}_{\mu}(u ; \Xi(h))$ в правой части неравенства Корна (произведение постоянной, не зависящей от параметров $h, \mu$ и поля смещений $u$, и какого-либо энергетического функционала; ср. далее определения (2.6), (2.56) и др.), но будем в левой части неравенства варьировать множители при квадратах $L_{2}$-норм смещений и их производных. При этом соболевские нормы можно делать анизотропными, адаптируя их к тонким телам и рассчитывая вывести асимптотически точные неравенства.

Анизотропные соболевские нормы были впервые использованы в неравенстве Корна, по-видимому, в статье [8], посвященной обоснованию теории пластин Кирхгофа. Затем в [9] и [10 ${ }^{1}$ были установлены весовые и анизотропные неравенства Корна для иилиндрических пластин и стержней, а в [12], [13] и [10], [14] они были перенесены на случаи слабоизогнутых или локалъно периодических тонких тел с переменным сечением (см. также книгу [15; гл. 3]).

Поясним необходимость анизотропного строения норм на простейшем примере - упругой балке, т. е. прямоугольнике $\Xi_{h}=(-1,1) \times(-h / 2, h / 2) \ni x=$ $\left(x_{1}, x_{2}\right)$ с малой толщиной $h$. Для скалярной функции $v \in H^{1}\left(\Xi_{h}\right)$, обращающейся в нуль на боковых (коротких) сторонах прямоугольника, неравенство Фридрихса выглядит следующим образом:

$$
\left\|v ; L_{2}\left(\Xi_{h}\right)\right\|^{2} \leqslant c\left\|\nabla_{x} v ; L_{2}\left(\Xi_{h}\right)\right\|^{2}
$$

причем множитель $c=\pi^{2} / 4$ не зависит от $h$. Если функция $v$ нечетна по переменной $x_{2}$, т. е. $v=0$ при $x_{2}=0$, то неравенство (1.1) улучшается, приобретая большой множитель в левой части, и принимает вид

$$
h^{-2}\left\|v ; L_{2}\left(\Xi_{h}\right)\right\|^{2} \leqslant c\left\|\nabla_{x} v ; L_{2}\left(\Xi_{h}\right)\right\|^{2}
$$

с той же постоянной $c=\pi^{2} / 4$. Оказывается, что такие простые соотношения вовсе не присущи неравенству Корна ${ }^{2}$.

ПримеР 1.1. Если торцы балки жестко защемлены, т. е. вектор смещений $u=\left(u_{1}, u_{2}\right)$ обращается в нуль при $x_{1}= \pm 1$, то асимптотически точное нера-

\footnotetext{
${ }^{1} \mathrm{~B}[10]$ оптимальное распределение весовых множителей в неравенстве Корна на тонком стержне достигнуто при помощи результатов [11].

${ }^{2}$ Примеры возникли в беседах автора с А. С. Слуцким и Г. А. Чечкиным.
} 
венство Корна выглядит следующим образом (ср. теорему 2.5):

$$
\begin{gathered}
\left\|u_{1} ; L_{2}\left(\Xi_{h}\right)\right\|^{2}+h^{2}\left\|u_{2} ; L_{2}\left(\Xi_{h}\right)\right\|^{2}+h^{2}\left\|\frac{\partial u_{1}}{\partial x_{2}} ; L_{2}\left(\Xi_{h}\right)\right\|^{2}+h^{2}\left\|\frac{\partial u_{2}}{\partial x_{1}} ; L_{2}\left(\Xi_{h}\right)\right\|^{2} \\
\leqslant c \mathscr{E}\left(u ; \Xi_{h}\right):=\sum_{j, k=1,2}\left\|\frac{\partial u_{j}}{\partial x_{k}}+\frac{\partial u_{k}}{\partial x_{j}} ; L_{2}\left(\Xi_{h}\right)\right\|^{2} \cdot
\end{gathered}
$$

Нормы производных $\partial u_{1} / \partial x_{1}$ и $\partial u_{2} / \partial x_{2}$ в пространстве Лебега $L_{2}\left(\Xi_{h}\right)$ фигурируют в правой части (1.2), представляющей собой эрзац функционала упругой энергии (см. далее п. 2.1), и поэтому не нуждаются в оценке. Если считать, что поле $u$ обладает симметрией относительно средней линии прямоугольника, а компоненты $u_{1}$ и $u_{2}$ являются соответственно четной и нечетной функциями переменной $x_{2}$, т. е. реализуется “чистое" растяжение балки $\Xi_{h}$, то неравенство Корна (1.2), как и неравенство Фридрихса (1.1), улучшается за счет устранения коэффициентов $h^{2}$ в левой части и принимает вид

$$
\left\|u_{1} ; L_{2}\left(\Xi_{h}\right)\right\|^{2}+h^{-2}\left\|u_{2} ; L_{2}\left(\Xi_{h}\right)\right\|^{2}+\left\|\frac{\partial u_{1}}{\partial x_{2}} ; L_{2}\left(\Xi_{h}\right)\right\|^{2}+\left\|\frac{\partial u_{2}}{\partial x_{1}} ; L_{2}\left(\Xi_{h}\right)\right\|^{2} \leqslant c \mathscr{E}\left(u ; \Xi_{h}\right) .
$$

Большой множитель $h^{-2}$ можно поставить и при первой норме в левой части (1.3) при дополнительном условии $u\left(x_{1}, 0\right)=0$. Однако в случае симметрии, обеспечивающей "чистый” изгиб (функции $u_{1}$ и $u_{2}$ - соответственно нечетная и четная относительно $\left.x_{2}\right)$, условие $u_{1}\left(x_{1}, 0\right)=0$ не привносит никаких изменений в неравенство Корна (1.2). Убеждаемся в этом, рассмотрев поле смещений с компонентами $u_{2}(x)=\left(x_{1}^{2}-1\right)^{2}$ и $u_{1}(x)=-4 x_{1} x_{2}\left(x_{1}^{2}-1\right)$.

ПримеР 1.2. Если балка свободна и никакие ограничения на поле смещений не накладываются, то справедливо такое неравенство Корна:

$$
\begin{aligned}
& h^{2}\left\|\frac{\partial u_{1}}{\partial x_{2}} ; L_{2}\left(\Xi_{h}\right)\right\|^{2}+h^{2}\left\|\frac{\partial u_{2}}{\partial x_{1}} ; L_{2}\left(\Xi_{h}\right)\right\|^{2} \\
& \quad \leqslant c\left(\mathscr{E}\left(u ; \Xi_{h}\right)+\left\|u_{1} ; L_{2}\left(\Xi_{h}\right)\right\|^{2}+h^{2}\left\|u_{2} ; L_{2}\left(\Xi_{h}\right)\right\|^{2}\right) .
\end{aligned}
$$

Его проверка проводится по той же схеме, что и далее доказательство теоремы 2.5. На первый взгляд кажется, что, убрав множитель $h^{2}$ при норме поперечного смещения $u_{2}$ (прогиба), можно добиться выполнения неравенства

$$
\begin{aligned}
& \left\|\frac{\partial u_{1}}{\partial x_{2}} ; L_{2}\left(\Xi_{h}\right)\right\|^{2}+\left\|\frac{\partial u_{2}}{\partial x_{1}} ; L_{2}\left(\Xi_{h}\right)\right\|^{2} \\
& \quad \leqslant \operatorname{ch}^{-\tau}\left(\mathscr{E}\left(u ; \Xi_{h}\right)+\left\|u_{1} ; L_{2}\left(\Xi_{h}\right)\right\|^{2}+\left\|u_{2} ; L_{2}\left(\Xi_{h}\right)\right\|^{2}\right)
\end{aligned}
$$

с показателем $\tau=0$ и тем самым полностью избавиться от присутствия малого параметра. Это впечатление ошибочно. Некоторая модификация доказательства той же теоремы 2.5 обеспечивает показатель $\tau=1$, а пример, подтверждающий точность неравенства, доставляет поле смещений с компонентами $u_{2}(x)=\left(\left(x_{1}^{2}-h\right)_{+}\right)^{2}$ и $u_{1}(x)=-4 x_{1} x_{2}\left(x_{1}^{2}-h\right)_{+}$, локализованное в $c \sqrt{h}$-окрестности центра балки и потому удовлетворяющее условию $u\left( \pm 1, x_{2}\right)=0$ защемления ее торцов. Здесь $t_{+}-$положительная часть $(t+|t|) / 2$ числа $t$. 
Необходимость включения в соболевские нормы весовых множителей проясняется при рассмотрении простейшей задачи о бесконечной консоли - полуполосе $\Pi=(0,+\infty) \times(-1 / 2,1 / 2)$ с защемленным торцом $\varpi=\{0\} \times(-1 / 2,1 / 2)$.

ПримеР 1.3. Пусть $u=\left(u_{1}, u_{2}\right) \in C_{c}^{\infty}(\bar{\Pi} \backslash \varpi)^{2}-$ гладкое поле смещений с компактным носителем, отделенным от торца $\varpi$. Под энергетическим пространством $\mathfrak{E}(\Pi)$ для задачи теории упругости в П естественно понимать пополнение линеала таких полей по энергетической норме $\mathscr{E}(u ; \Pi)^{1 / 2}$. Это пространство содержит вектор-функции $u \in H_{\mathrm{loc}}^{1}(\bar{\Pi})^{2}$, обращающиеся в нуль на $\varpi$, однако не совпадает с пространством Соболева $u \in \stackrel{\circ}{H^{1}}(\Pi ; \varpi)^{2}$ (символ ${ }^{\circ}$ указывает на условие $\left.u\left(x_{1}, 0\right)=0\right)$. Дело в том, что так называемые жесткие смещения - переносы $\left(c_{1}, c_{2}\right)$ и повороты $c_{0}\left(-x_{2}, x_{1}\right)$ - можно приблизить по энергетической норме полями из $C_{c}^{\infty}(\bar{\Pi})^{2}$. Упомянем статьи [16], [17], в которых изучаются энергетические классы в параболоидальных, в частности цилиндрических, областях и отслеживается их связь с классическим принципом Сен-Венана. Для полуполосы неравенство Корна выглядит так:

$$
\begin{gathered}
\left\|\left(1+x_{1}^{2}\right)^{-1} u_{1} ; L_{2}(\Pi)\right\|^{2}+\left\|\left(1+x_{1}^{2}\right)^{-2} u_{2} ; L_{2}(\Pi)\right\|^{2}+\left\|\left(1+x_{1}^{2}\right)^{-1} \frac{\partial u_{1}}{\partial x_{2}} ; L_{2}(\Pi)\right\|^{2} \\
+\left\|\left(1+x_{1}^{2}\right)^{-1} \frac{\partial u_{2}}{\partial x_{1}} ; L_{2}(\Pi)\right\|^{2} \leqslant c \mathscr{E}(u ; \Pi)
\end{gathered}
$$

(см. [7], [9], [18]). В сравнении с неравенством Корна (1.2) в весовом неравенстве (1.4) вместо малого коэффициента $h$ появился множитель $\left(1+x_{1}^{2}\right)^{-1}$, исчезающий на бесконечности. Примеры показывают, что ни один из весов нельзя увеличить.

Растяжение координат $x \mapsto \xi=\left(h^{-1}\left( \pm x_{1}+1\right), h^{-1} x_{2}\right)$ переводит балку $\Xi_{h}$ в длинный прямоугольник $\left(0,2 h^{-1}\right) \times(-1 / 2,1 / 2)$, а поле $u$, аннулирующееся на торцах балки, продолжается нулем на всю полосу. Применив неравенство (1.4), уточняем неравенство Корна (1.2) в тонкой области $\Xi_{h}$ путем введения весовых множителей, возрастающих при $x_{1} \rightarrow \pm 1$ :

$$
\begin{aligned}
\left\|\left(x_{1}^{2}+h^{2}\right)^{-1} u_{1} ; L_{2}\left(\Xi_{h}\right)\right\|^{2}+h^{2}\left\|\left(x_{1}^{2}+h^{2}\right)^{-2} u_{2} ; L_{2}\left(\Xi_{h}\right)\right\|^{2} \\
+h^{2}\left\|\left(x_{1}^{2}+h^{2}\right)^{-1} \frac{\partial u_{1}}{\partial x_{2}} ; L_{2}\left(\Xi_{h}\right)\right\|^{2} \\
+h^{2}\left\|\left(x_{1}^{2}+h^{2}\right)^{-1} \frac{\partial u_{2}}{\partial x_{1}} ; L_{2}\left(\Xi_{h}\right)\right\|^{2} \leqslant c \mathscr{E}\left(u ; \Xi_{h}\right)
\end{aligned}
$$

(см. [9], [10] и далее теоремы 2.6, 2.9 для пластин и стержней). Зачастую множители $\left(x_{1}^{2}+h^{2}\right)^{-1}$ и подобные им имеют решающее значение при выводе неулучшаемых неравенств Корна для мультиструктур и обосновании асимптотических конструкций для упругих полей.

Весовые анизотропные нормы оказались наиболее приспособленными к отслеживанию зависимостей упругих полей от малых и больших геометрических параметров. Показательным в этом плане является упругое сочленение типа тело/стержни ("кактус"), подробно обсуждаемое далее в 33 и определенное формулой (3.1). Вывод неравенства Корна для подобных однородных $(\mu=1)$ 
сочленений $\Xi(h)$, закрепленных по объединению $\Gamma(h)$ удаленных от тела торцов стержней, имеет долгую историю. Так, в [19] в общем случае и в [20], [21] при дополнительном условии "треугольника" (см. геометрическое требование $2^{\circ}$ ) в п. 2.2) были установлены неравенства Корна, которые, в частности, обеспечивали следующую оценку для нормы смещений на теле $\Xi$ без стержней:

$$
h^{m}\left\|u ; H^{1}(\Xi)\right\|^{2} \leqslant c \mathscr{E}(u ; \Xi(h)), \quad u \in \stackrel{\circ}{H^{1}}(\Xi(h) ; \Gamma(h))^{3} .
$$

При этом $m=10$ в [19] и $m=8$ в [20], [21]. В работе [22] при том же условии, что и в [20], показатель $m$ в левой части (1.6) был уменьшен до 4 , но затем в [23] окончательно установлено равенство $m=4$ без каких-либо ограничений, т. е. при рассмотрении однородного сочленения условие “треугольника" оказалось ненужным. Кроме того, в [22] при более сильном требовании “заполнения пространства $\mathbb{R}^{6}$ " (см. условие $1^{\circ}$ ) в п. 2.2 и п. 3.1) было показано, что постоянная Корна $C(\Xi(h))$ не превосходит $c h^{-2}(1+|\log h|)^{2}$, но затем в работе [24] логарифмический множитель был убран и было окончательно установлено, что при удачном расположении шести стержней в неравенстве (1.6) можно взять $m=2$. Далее в $\S 3$ приводятся примеры, подтверждающие, что названные показатели $m=4$ и $m=2$ уменьшить нельзя. Подчеркнем, что норма в левой части (1.6) изотропная лишь по причине отсутствия в оценке норм смещений на тонких стержнях.

Все приведенные далее весовые анизотропные неравенства Корна для различных типов сочленений являются неулучшаемыми и асимптотически точными относительно обоих параметров $h \in\left(0, h_{0}\right]$ и $\mu>0$, а распределение весовых множителей - оптимальным. Это подтверждено примерами конкретных конструкций, поясненных рисунками. Исключение составляет множитель $(1+|\log h|)^{-2}$, возникающий при определенных соотношениях между предельными размерностями и наследуемый от неравенства Харди "с логарифмом" (см. далее формулы (2.27) и (2.26)). В примере 2.3 проверено, что какая-то степень логарифма все-таки необходима, однако убедиться в точности показателя -2 автору не удалось. Впрочем, неприятности с логарифмами отмечены также в статьях [7] и [16], но вопрос о правильности их включения в весовые оценки до сих пор остается открытым.

1.3. Технические приемы. Несмотря на достаточную общность изучаемых геометрических форм и конфигураций, можно придумать сочленения, не охваченные формулируемыми в обзоре утверждениями. Вместе с тем, предлагаемые методы исследования имеют более широкую сферу применения и, разумеется, представленные далее результаты допускают всевозможные обобщения. Поэтому весьма важным представляется выявление и перечисление технических приемов и трюков, обеспечивающих асимптотическую точность получаемых весовых анизотропных неравенств Корна. Разумеется, отправной точкой исследований всегда остаются неравенства Корна для одиночных (изолированных) элементов конструкций - массивных тел, тонких пластин и стержней (см. предложение 2.1 и теоремы 2.1, 2.7, 2.9), однако для сопряжения этих неравенств требуются новые идеи и подходы, а возникающие в конечном итоге весовые нормы зачастую существенно отличаются от начальных. 
Приведем список основных приемов и укажем разделы обзора, содержащие их упоминание и ссылки на статьи с детальным описанием.

1) Распространение неравенства Корна на обгемлющее множество: отщепление жесткого смещения и продолжение поля смещений с сохранением оценки функционала энергии или весовой анизотропной нормы (пп. 2.3, 2.4, $2.6-2.8,3.5,4.5,5.2,5.3$ и 7.3$)$.

2) Отщепление жесткого смещения и оченка элементов соответствующего столбца $b \in \mathbb{R}^{6}$ (см., например, представление $\left.\left(2.58_{1}\right)\right)$ путем составления системы алгебраических уравнений (пп. 2.2, 2.5, 3.2-3.4, 4.2, 5.2, 6.2, 6.3, 7.4).

3) Перенос начала декартовой системы координат при оценке элементов столби, $b$ (пп. 2.5, 3.3, 2.4, 5.4, 5.5, 6.3, 7.3-7.5).

4) Обработка составляющих разложений поля смещений на мальх множествах при помощи вариантов неравенства Харди (пп. 2.2, 2.5, 3.2-3.4, 4.2, 4.3, $5.2,5.8,6.2,7.3)$.

5) Обработка средних поворотов и крутильной составляющей поля смещений на тонком стержне при помощи неравенства Пуанкаре (пп. 2.6, 3.3 и 4.2), а также лемма 4.1, доставляющая оченку интеграла (4.6) (пп. 4.2 и 6.2).

6) Перенос оченки нормы смещений с одного на другое, близко расположенное, тонкое тело (пп. 5.6, 6.2, 6.3, 7.3-7.5).

7) Конструкиия вспомогательного искусственного тонкого обгекта (процедура "tetris") (п. 2.8).

8) Классификация элементов конструкиии (процедура "риzzle") (п. 7.6).

1.4. Структура статьи. Обзор начинается с перечисления в $\S 2$ неравенств Корна для одиночных тел - массивных и тонких. Для последних, т.е. для пластин и стержней, используются весовые анизотропные соболевские нормы, а прием перенесения неравенства Корна на объемлющее множество позволяет обслужить не только цилиндрические, но и слабо искривленные или локально периодические тонкие тела. В п. 2.7 рассматриваются составные тела с контрастными жесткостями. Кроме того, выводятся неравенства Корна для тел и пластин, закрепленных по малым множествам или имеющих малые дефекты (пп. 2.2, 2.3 и 2.5). Здесь впервые возникают условие "заполнения пространства $\mathbb{R}^{6}$ " и условие "треугольника"; они или их двумерные аналоги играют решающую роль и во всех остальных параграфах обзора, где исследуются сами упругие сочленения.

Третий параграф посвящен сочленениям типа тело/стержни, причем при контрастных жесткостях различаются случаи пронизывающих стержней и стержней, вставленных в неглубокие гнезда. Поскольку торцы стержней $G_{h}^{1}, \ldots, G_{h}^{J}$, выступающие из тела, защемлены, анизотропные нормы |·; $G_{h}^{j} \mathbf{|}$ предопределены теоремой 2.9, но норма на массивном теле, закрепленном только посредством стержней, существенно зависит как от параметров $h$ и $\mu$, так и от конфигурации стержней. Интересно то, что при различных соотношениях между параметрами $h$ и $\mu$ оптимальному расположению стержней отвечают разные геометрические требования $1^{\circ}$ ) или $2^{\circ}$ ), уже упоминавшиеся выше. В последнем п. 3.5 рассматривается наиболее простая ситуация: жестко защемлена часть поверхности тела $\Xi$, к которому присоединены стержни $G_{h}^{1}, \ldots, G_{h}^{J}$ со свободными концами. 
В $\S 4$ исследуются сочленения массивного тела с тонкими пластинами. Как и в предыдущем параграфе, приходится различать пластины $\widetilde{\Omega}_{h}^{1}, \ldots, \widetilde{\Omega}_{h}^{J}$, вставленные в мелкие пазы, и пластины $\Omega_{h}^{1}, \ldots, \Omega_{h}^{J}$, вставленные в глубокие прорези. Опять-таки благодаря защемлению участков боковых поверхностей нормы $\mathbf{I} \cdot ; \widetilde{\Omega}_{h}^{j} \mathbf{I}_{0}$ и $\mathbf{|} \cdot ; \Omega_{h}^{j} \mathbf{I}_{0}, j=1, \ldots, J$, назначаются теоремами 2.7 и 2.6 , а норма на поддерживаемом теле $\Xi$ определяется в соответствии со значениями параметров $h, \mu$ и расположением пластин. Наглядная интерпретация накладываемых геометрических условий дана в леммах 4.2 и 4.3. В последнем пункте параграфа обсуждается конструкция, состоящая из закрепленного тела и присоединенной к нему пластины.

Поскольку пластина имеет одно ослабленное (поперечное) направление, а стержень - два, сочленения типа пластина/стержни, исследуемые в $\S 5$, оказываются наиболее сложными для анализа и количество вариантов взаимного расположения элементов конструкции огромно. Поэтому в п. 2 приводятся оценки для компонент среднего жесткого смещения на пластине, необходимые для формирования весового анизотропного неравенства Корна, а затем рассматриваются несколько случаев: один или два стержня, шесть стержней, удовлетворяющих условию заполнения пространства $\mathbb{R}^{6}$, или три стержня, обеспечивающих оптимальное крепление пластины в продольных или поперечном направлениях. В пп. 5.7 и 5.8 изучаются закрепление пластины параллельными пронизывающими стержнями и жесткостные свойства стержней, присоединенных к пластине, которая защемлена вдоль боковой поверхности.

Последние два параграфа посвящены сочленениям однотипных тонких тел соответственно пластин и стержней. В $\S 6$ постановка задачи - та же, что в предыдущих четырех параграфах: положение одной из пластин $\Omega_{h}^{0}$ зафиксировано при помощи нескольких других $\Omega_{h}^{1}, \ldots, \Omega_{h}^{J}$ (исключение составляет п. 6.5, где продемонстрировано, как можно изучить достаточно произвольные тонкостенные конструкции). Приведены результаты того же свойства в неравенстве Корна подбирается анизотропная норма ||$\cdot ; \Omega^{0}(h) \mid \boldsymbol{I}_{\beta}$, при которой неравенство становится асимптотически точным, и приводятся примеры конкретных конструкций, подтверждающие эту точность. Помещенные в $\S 7$ результаты статьи [25] имеют иное качество: изучаются произвольные системы тонких стержней и предлагается алгорифм, позволяющий классифицировать элементы конструкции и вывести асимптотически точное неравенство Корна для всего сочленения. Подчеркнем, что в механике такая классификация не была известна.

1.5. Обозначения. Пометка $[N]$ перед формулировкой утверждения означает, что его доказательство можно найти в публикации $[N]$. При отсутствии пометки утверждения снабжены пояснениями. Для теорем 2.5 и 3.1, 3.2, содержащих принципиально важные результаты для анализа тонких упругих тел и их сочленений, приведены краткие выкладки, обеспечивающие их проверку.

Все возникающие далее постоянные в неравенствах, если не оговорено противное, не зависят от параметров $h \in\left(0, h_{0}\right], \mu>0$ и, разумеется, от фигурирующих в них функции или вектор-функции. 


\section{§ 2. Неравенства Корна для одиночных тел}

2.1. Массивное тело. Пусть $\Xi \in \mathbb{R}^{3}$ - область с компактным замыканием $\bar{\Xi}$, представимая как объединение конечного числа силъно липшицевых областей. Последние характеризуются тем, что каждая точка на границе $\partial \Xi$ имеет окрестность, в которой область задана неравенством $y_{3}>\varphi\left(y^{\prime}\right)$, где $y^{\prime}=\left(y_{1}, y_{2}\right), y=\left(y^{\prime}, y_{3}\right)$ - подходящая система декартовых координат, а $\varphi-$ функция, подчиненная условию Липшица $\left|\varphi\left(y^{\prime}\right)-\varphi\left(\eta^{\prime}\right)\right| \leqslant M\left|y^{\prime}-\eta^{\prime}\right|$ с общей для всех точек константой $M$. Всякая сильно липшицева область удовлетворяет условию конуса: любую точку $x \in \bar{\Xi}$ можно совместить с вершиной усеченного кругового конуса $\mathbb{K}(H, R) \in \Xi$ с фиксированными, но зависящими от $\Xi$ высотой $H>0$ и радиусом основания $R>0$.

В качестве $\Xi$ может выступать любая область с кусочно гладкой границейдопускаются конические точки и ребра, гладкие или пересекающиеся. На первый взгляд, неприятности привносятся трещинами, т. е. ребрами с раствором угла $2 \pi$, поскольку задать область при помощи упоминавшегося выше неравенства не удается. Тем не менее, разрезав тело вдоль продолжений трещин, все-таки получаем сильно липшицевы области. Нетрудно построить невыпуклый конус, для которого требуемое условие соблюдается только после рассечения на части. Примерами областей, не удовлетворяющих условию Липшица по существу, служат множества с нулевыми заострениями

$$
\begin{aligned}
& \Xi^{1}=\left\{x=\left(x^{\prime}, x_{3}\right): x_{3} \in(0,1), x_{3}^{-\gamma} x^{\prime} \in \omega\right\}, \\
& \Xi^{2}=\left\{x: x^{\prime}=\left(x_{1}, x_{2}\right) \in \omega,\left|x_{3}\right|<H\left(x^{\prime}\right)\right\} .
\end{aligned}
$$

Здесь $\gamma>1, \omega$ - область на плоскости $\mathbb{R}^{2}$, ограниченная простым гладким замкнутым контуром $\partial \omega$, а $H$ - гладкая положительная на $\omega$ функция, причем $H\left(x^{\prime}\right)=H_{0} \operatorname{dist}\left(x^{\prime}, \partial \omega\right)^{\gamma}$ вблизи контура $\partial \omega$ и $H_{0}>0$.

Зафиксируем какую-либо декартову систему координат $x$. Если $u_{j}(x)-$ декартовы компоненты вектора $u(x)$ смещения точки $x \in \Xi$ под действием каких-либо внешних сил, то в рамках линейной теории упругости деформированное состояние тела характеризуется тензором деформаций с декартовыми компонентами

$$
\varepsilon_{j k}(u ; x)=\frac{1}{2}\left(\frac{\partial u_{j}}{\partial x_{k}}(x)+\frac{\partial u_{k}}{\partial x_{j}}(x)\right), \quad j, k=1,2,3 .
$$

Упругая энергия, запасенная телом $\Xi$, вычисляется по формуле

$$
\sum_{j, k=1}^{3} \sum_{p, q=1}^{3} \int_{\Xi} a_{j k, p q}(x) \varepsilon_{j k}(u ; x) \varepsilon_{p q}(u ; x) d x
$$

где $a_{j k, p q}(x)$ - упругие модули материала в точке $x \in \Xi$, составляющие четырехвалентный тензор Гука, который обладает обычными свойствами симметрии и положительной определенности (см., например, [26], [27]). Уточнение сказанного здесь не требуется, так как для целей настоящей статьи достаточно иметь дело с “эрзацем" упругой энергии $\mathscr{E}(u ; \Xi)=\sum \int\left|\varepsilon_{j k}(u ; x)\right|^{2} d x$ (суммирование
по $j, k=1,2,3$ и интегрирование по $x \in \Xi)$. 
Далее удобно использовать матричную, а не тензорную форму записи (см. [26], а также [15] и др.); она предложена С. Г. Лехницким в 30-х годах прошлого столетия. Вектор смещений интерпретируется как столбец $u=$ $\left(u_{1}, u_{2}, u_{3}\right)^{\top}$ высотой 3 , и вводится столбеи, дебормаций

$$
\varepsilon(u)=\left(\varepsilon_{11}(u), \varepsilon_{22}(u), \varepsilon_{33}(u), \alpha^{-1} \varepsilon_{23}(u), \alpha^{-1} \varepsilon_{31}(u), \alpha^{-1} \varepsilon_{12}(u)\right)^{\top} .
$$

Здесь ${ }^{\top}$ - знак транспонирования, а множитель $\alpha=2^{-1 / 2}$ уравнивает естественные нормы симметричного двухвалентного тензора с компонентами $(2.2)$ и шестимерного столбца (2.4). Связь $\varepsilon(u)=D\left(\nabla_{x}\right) u$ столбцов смещений и деформаций теперь реализуется при помощи $(6 \times 3)$-матрицы $D\left(\nabla_{x}\right)$ дифференциальных операторов первого порядка,

$$
D(x)^{\top}=\left(\begin{array}{cccccc}
x_{1} & 0 & 0 & 0 & \alpha x_{3} & \alpha x_{2} \\
0 & x_{2} & 0 & \alpha x_{3} & 0 & \alpha x_{1} \\
0 & 0 & x_{3} & \alpha x_{2} & \alpha x_{1} & 0
\end{array}\right), \quad \partial_{j}=\frac{\partial}{\partial x_{j}}, \quad \alpha=\frac{1}{\sqrt{2}} .
$$

Выполняется равенство

$$
\mathscr{E}(u ; \Xi)=\left\|D\left(\nabla_{x}\right) u ; L_{2}(\Xi)\right\|^{2}=\int_{\Xi}\left|D\left(\nabla_{x}\right) u(x)\right|^{2} d x .
$$

Нетрудно убедиться в том, что для гладкого поля $u \in C^{\infty}(\bar{\Xi})^{3}$ справедливо следующее высказывание:

$$
D\left(\nabla_{x}\right) u(x)=0 \in \mathbb{R}^{6} \text { при } x \in \Xi \quad \Longleftrightarrow u(x)=d(x) b .
$$

Здесь $b \in \mathbb{R}^{6}$ - какой-либо столбец, а $d$ - линейная матрица-функция размером $3 \times 6$,

$$
d(x)=\left(d^{\prime}, d^{\prime \prime}(x)\right)=\left(\begin{array}{cccccc}
1 & 0 & 0 & 0 & \alpha x_{3} & -\alpha x_{2} \\
0 & 1 & 0 & -\alpha x_{3} & 0 & \alpha x_{1} \\
0 & 0 & 1 & \alpha x_{2} & -\alpha x_{1} & 0
\end{array}\right)
$$

ЗАмечАниЕ 2.1. Матрицы $D$ и $d$ из (2.5) и (2.8) удовлетворяют соотношениям

$$
\begin{aligned}
D\left(\nabla_{x}\right) D(x)^{\top} & =\mathbb{I}_{6}, & D\left(\nabla_{x}\right) d(x) & =\mathbb{O}_{6}, \\
\left.d\left(\nabla_{x}\right)^{\top} D(x)^{\top}\right|_{x=0} & =\mathbb{O}_{6}, & \left.d\left(\nabla_{x}\right)^{\top} d(x)\right|_{x=0} & =\mathbb{I}_{6},
\end{aligned}
$$

в которых $\mathbb{I}_{n}$ и $\mathbb{O}_{n}-$ единичная и нулевая матрицы размером $n \times n$.

В правой части формулы (2.7) стоит жесткое смещение, причем $d^{\prime} b^{\prime}-$ поступательное, а $d^{\prime \prime}(x) b^{\prime \prime}$ - вращательное (относительно начала координат $\mathscr{O}$ ). Укороченные столбцы $b^{\prime}=\left(b_{1}, b_{2}, b_{3}\right)^{\top}$ и $b^{\prime \prime}=\left(b_{4}, b_{5}, b_{6}\right)^{\top}$ соответствуют разбиению матрицы $d(x)$ на $(3 \times 3)$-блоки $d^{\prime}(x)$ и $d^{\prime \prime}(x)$, произведенному в средней части формулы (2.8). Далее часто будет востребован линеал жестких смещений $\mathfrak{R}=\left\{u(x)=d(x) b: b \in \mathbb{R}^{6}\right\}$. Он, а также функционалы (2.3) и (2.6), разумеется, не зависят от выбора системы координат $x$. Формулы для преобразования рассматриваемых объектов при замене декартовых координат приведены в следующей лемме (см., например, [22] и [15; § 2.1]). 
Лемма 2.1. При замене координат $x \mapsto \mathbf{x}=\Theta\left(x-x^{0}\right)$, где $\Theta$ - ортогональная $(3 \times 3)$-матрица, жесткое смешение $d(x) b$ сохраняет вид $d(\mathbf{x}) \mathbf{b}$, причем $\mathbf{b}^{\prime}=\Theta d\left(x^{0}\right) b u \mathbf{b}^{\prime \prime}=\Theta b^{\prime \prime}$.

Отметим, что благодаря коэффициентам $\alpha^{-1}=\sqrt{2}$, отсутствующим в [26] и введенным в определение (2.4) в [15], преобразования столбцов деформаций и напряжений также осуществляются при помощи ортогональных матриц.

Приведенное ниже неравенство Корна доказывалось разными способами многими математиками (см. в работах [1]-[6] и др.). По мнению автора, наиболее простое и изящное доказательство дано В. А. Кондратьевым и О.А. Олейник в статье [7].

ТЕОРема 2.1. При указанных в начале пункта ограничениях на область для всякого поля $u \in H^{1}(\Xi)^{3}$ выполнено неравенство

$$
\left\|\nabla_{x} u ; L_{2}(\Xi)\right\|^{2} \leqslant c_{\Xi}\left(\mathscr{E}(u ; \Xi)+\left\|u ; L_{2}(\Xi)\right\|^{2}\right),
$$

в котором множитель $c_{\Xi}$ зависит от области $\Xi$, но не от поля и.

Благодаря названному свойству постоянной $c_{\Xi}$ пространство $\mathfrak{E}(\Xi)=\{u \in$ $\left.L_{2}(\Xi)^{3}: \mathscr{E}(u ; \Xi)<\infty\right\}$, которое можно получить пополнением линеала $C^{\infty}(\bar{\Xi})^{3}$ по энергетической норме $\|u ; \mathfrak{E}(\Xi)\|:=\left(\mathscr{E}(u ; \Xi)+\left\|u ; L_{2}(\Xi)\right\|^{2}\right)^{1 / 2}$, не отличается ни алгебраически, ни топологически от пространства Соболева $H^{1}(\Xi)^{3}$. Покажем, что без требования липшицевости области этот факт неверен.

ПримеР 2.1. 1) Для поля смещений $u$ с компонентами

$$
u_{i}(x)=w_{i}\left(x_{3}\right), \quad i=1,2, \quad u_{3}(x)=-x_{1} \partial_{3} w_{1}\left(x_{3}\right)-x_{2} \partial_{3} w_{2}\left(x_{3}\right)
$$

по формулам (2.2) находим деформации $\varepsilon_{11}(u)=\varepsilon_{22}(u)=\varepsilon_{23}(u)=\varepsilon_{31}(u)=$ $\varepsilon_{12}(u)=0$ и $\varepsilon_{33}(u ; x)=-x_{1} \partial_{3}^{2} w_{1}\left(x_{3}\right)-x_{2} \partial_{3}^{2} w_{2}\left(x_{3}\right)$. Прямыми вычислениями проверяем, что при $w_{i}\left(x_{3}\right)=a_{i} x_{3}^{\beta}, a_{i}>0, \beta \in \mathbb{R}$, сходимость интегралов $\mathscr{E}\left(u ; \Xi^{1}\right),\left\|u ; L_{2}\left(\Xi^{1}\right)\right\|^{2}$ и $\left\|\nabla_{x} u ; L_{2}\left(\Xi^{1}\right)\right\|^{2}$ по пикообразной области $\left(2.1_{1}\right)$ имеет место соответственно при таких условиях: $\beta>-2 \gamma+3 / 2, \beta>-\gamma-1 / 2$ и $\beta>$ $-\gamma+1 / 2$. В случае $\gamma>1$ интервал $(\max \{-2 \gamma-3 / 2,-\gamma-1 / 2\},-\gamma+1 / 2) \ni \beta$ не пустой, а значит, при таких показателях $\beta$ для поля (2.10) энергетическая норма $\|u ; \mathfrak{E}(\Xi)\|$ конечна, а норма $\left\|u ; H^{1}(\Xi)\right\|^{2}$ нет. Более того, если $\gamma>2$ и $\beta+\gamma+1 / 2 \in(2-\gamma, 0)$, то $u \neq L_{2}(\Xi)^{3}$, но ${ }^{3} \mathscr{E}(u ; \Xi)<\infty$.

2) В предположении конечности всех слагаемых неравенство (2.9) для пикоообразной области также неверно. Положим $w_{i}(x)=a_{i} \chi_{\infty}\left(\log x_{3}+\log N\right) x_{3}^{\beta}, i=$ 1,2 , в определении (2.10); при этом $N \in \mathbb{N}:=\{1,2, \ldots\}$ и $\chi_{\infty} \in C_{0}^{\infty}(\mathbb{R})$ - такая срезающая функция, что $\chi_{\infty}(t)=0$ при $t<1$ и $\chi_{\infty}(t)=1$ при $t>2$. Включение $u \in C^{\infty}\left(\overline{\Xi^{1}} \backslash\{0\}\right)^{3}$ очевидно, так как вектор-функция $u$ равна нулю в окрестности вершины пика. Правая часть (2.9) составляет $O\left(N^{-\max \{0, \gamma-2\}-\gamma-\beta-1 / 2}\right)$, а левая $-O\left(N^{\gamma-\beta+1 / 2}\right)$, т. е. неравенство Корна с постоянной, не зависящей от поля $u$, невозможно.

3) Предшествующие рассуждения сохраняются и для “летающей тарелки" $\left(2.1_{2}\right)$, если положить $u_{3}(x)=w\left(x^{\prime}\right), u_{i}(x)=-x_{3} \partial_{i} w\left(x^{\prime}\right), i=1,2$, и взять

\footnotetext{
${ }^{3}$ Из-за потери компактности вложением $\mathfrak{E}(\Xi) \subset L_{2}(\Xi)^{3}$ в пикообразной области с показателем заострения $\gamma \geqslant 2$ спектр оператора задачи теории упругости не может быть дискретным (ср. [28; теорема 9.2.1]).
} 
$w\left(x^{\prime}\right)=\rho\left(x^{\prime}\right)^{\beta}$, где $\rho \in C^{\infty}(\bar{\omega})$ - положительная гладкая функция, эквивалентная расстоянию до контура $\partial \omega$.

Очередное утверждение, проверку которого можно найти, например, в книгах [4], [6], [29], [15], указывает заменитель для нормы $\left\|u ; L_{2}(\Xi)\right\|$ в правой части неравенства (2.9).

ПредлОЖЕНИЕ 2.1. Пусть $F=\left(F_{1}, \ldots, F_{6}\right)^{\top}$ - линейный функционал на $H^{1}(\Omega)^{3} u$

$$
\begin{aligned}
u^{n} \rightarrow u^{\infty} \text { слабо в } H^{1}(\Omega)^{3} & \Rightarrow F\left(u^{n}\right) \rightarrow F\left(u^{\infty}\right) \in \mathbb{R}^{6}, \\
F(r)=0 \text { при } r \in \Re & \Rightarrow r=0 \in \mathbb{R}^{6} .
\end{aligned}
$$

Тогда в условиях теоремы 2.1 выполняется неравенство

$$
\left\|\nabla_{x} u ; L_{2}(\Xi)\right\|^{2} \leqslant c\left(\mathscr{E}(u ; \Xi)+|F(u)|^{2}\right)
$$

c постоянной с, зависящей лищь от области $\Xi и$ функиионала $F$.

Обращаем внимание на то, что $\operatorname{dim} \mathfrak{R}=6$. Один из подручных инструментов в статье - неравенство (2.12) с подходящим функционалом $F$. Например, если $\Gamma \subset \partial \Xi, \operatorname{mes}_{2} \Gamma>0$ и $u \in \stackrel{\circ}{H^{1}}(\Xi ; \Gamma)^{3}$, причем символ ${ }^{\circ}$ указывает на краевое условие

$$
u(x)=0, \quad x \in \Gamma,
$$

то разумно использовать функционал

$$
F(u)=\int_{\Gamma} d(x)^{\top} u(x) d s_{x} \in \mathbb{R}^{6},
$$

уничтожаемый в правой части (2.12) упомянутым условием. В (2.14) и далее $d s_{x}$ - элемент площади поверхности. Отметим, что при жестком смещении $r=d c \in \mathfrak{R}$ справедливы формулы

$$
\begin{gathered}
F(r)=\int_{\Gamma} d(x)^{\top} r(x) d s_{x}=\mathbf{d}(\Gamma) c \\
\mathbf{d}(\Gamma)=\int_{\Gamma} d(x)^{\top} d(x) d s_{x} .
\end{gathered}
$$

Матрица $\mathbf{d}(\Gamma)$ размером $6 \times 6$ является матрицей Грама, симметрической и положительно определенной, так как столбцы матрицы (2.8) линейно независимы в пространстве $L_{2}(\Gamma)^{3}$. Значит, благодаря вложению $H^{1}(\Xi) \subset L_{2}(\Gamma)$ требования (2.11) выполнены для функционала $(2.14)$, и поле $u \in \stackrel{\circ}{H^{1}}(\Xi ; \Gamma)^{3}$ удовлетворяет соотношениям $c_{0}\left\|u ; H^{1}(\Xi)\right\|^{2} \leqslant\left\|\nabla_{x} u ; L_{2}(\Xi)\right\|^{2} \leqslant c \mathscr{E}(u ; \Xi)$. В левой части использовано неравенство Фридрихса-Пуанкаре, а множители $c_{0}>0$ и $c$ зависят лишь от множеств $\Xi$ и $Г$.

В частном случае $\Gamma=\partial \Xi$ продолжение поля $u \in \stackrel{\circ}{H}^{1}(\Xi)^{3}:=\stackrel{\circ}{H^{1}}(\Xi ; \partial \Xi)^{3}$ нулем на дополнение $\mathbb{R}^{3} \backslash \bar{\Xi}$, преобразование Фурье и равенство Парсеваля приводят к соотношению

$$
\left\|\nabla_{x} u ; L_{2}(\Xi)\right\|^{2} \leqslant 2\left\|D\left(\nabla_{x}\right) u ; L_{2}(\Xi)\right\|^{2}=2 \mathscr{E}(u ; \Xi) .
$$


Точные константы в неравенстве Корна известны лишь для областей немногих канонических форм (см. [30]-[32]). Для отслеживания зависимостей констант Корна от разнообразных геометрических параметров весьма полезен следующий результат, заимствованный из работы [7] и относящийся к областям $\Xi$, звездным относительно шара $\mathbb{B}_{R}=\{x:|x|<R\}$. Такое свойство означает, что отрезок, соединяющий произвольные точки в множествах $\Xi$ и $\mathbb{B}_{R}$, лежит целиком в $\Xi$, и, кроме того, оно гарантирует сильную липшицевость области $\Xi$. Любая сильно липшицева область может быть представлена как объединение конечного числа областей, звездных относительно некоторых шаров (см., например, [33; лемма 1.8.1]).

ТеОрема 2.2. Пусть $\Xi$-область, звездная относительно шара $\mathbb{B}_{R}$, и $2 \mathscr{R}$ диаметр этой области. Тогда для поля $u \in H^{1}(\Xi)^{3}$ выполняются неравенства

$$
\begin{aligned}
\left\|\nabla_{x} u ; L_{2}(\Xi)\right\|^{2} & \leqslant C_{1} \mathscr{R}^{3} R^{-3}\left(\mathscr{E}(u ; \Xi)+\left\|\nabla_{x} u ; L_{2}\left(\mathbb{B}_{R}\right)\right\|^{2}\right), \\
\mathscr{R}^{-2}\left\|u ; L_{2}(\Xi)\right\|^{2} & \leqslant C_{2} \mathscr{R} R^{-1}\left(\left\|\nabla_{x} u ; L_{2}(\Xi)\right\|^{2}+R^{-2}\left\|u ; L_{2}\left(\mathbb{B}_{R}\right)\right\|^{2}\right),
\end{aligned}
$$

в которых $C_{1}$ и $C_{2}-$ абсолютные константы.

Совмещая неравенства $\left(2.16_{1}\right)$ и $\left(2.16_{2}\right)$, приходим к соотношению

$$
\begin{aligned}
\|u ; \Xi\|_{\mathscr{R}}^{2} & :=\left\|\nabla_{x} u ; L_{2}(\Xi)\right\|^{2}+\mathscr{R}^{-2}\left\|u ; L_{2}(\Xi)\right\|^{2} \\
& \leqslant C_{3} \mathscr{R}^{4} R^{-4}\left(\mathscr{E}(u ; \Xi)+R^{-2}\left\|u ; L_{2}\left(\mathbb{B}_{R}\right)\right\|^{2}\right) .
\end{aligned}
$$

Оценки $\left(2.16_{1}\right),\left(2.16_{2}\right)$ точны в следующем смысле: в их мажоранты нельзя добавить множители, бесконечно малые при $R / \mathscr{R} \rightarrow 0$. Комбинированная оценка (2.17) теряет это свойство; впрочем, она и сами оценки $\left(2.16_{1}\right),\left(2.16_{2}\right)$ будут применяться в ситуации $\mathscr{R}=O(R)$. При таких $R$ требование звездности не нужно: достаточно, чтобы область $\Xi$ была сильно липшицевой. Неравенство $\left(2.16_{2}\right)$ верно и для области $\Xi$, звездной только относительно центра шара $\mathbb{B}_{R}$.

2.2. Массивное тело, закрепленное по малым множествам. Зафиксируем на поверхности $\partial \Xi$ точки $P^{1}, \ldots, P^{J}$ и обозначим $\Gamma_{h}^{j}$ пересечение $\partial \Xi \cap$ $\mathbb{B}_{h}\left(P^{j}\right)$; здесь $j=1, \ldots, J, \mathbb{B}_{h}(P)=\{x:|x-P|<h\}-$ шар и $h$ - малый геометрический параметр. Правильно говорить о малом параметре $h / \mathscr{R}$, однако масштабированием диаметр $2 \mathscr{R}$ области можно сделать единичным, а параметр $h>0$ - безразмерным.

На $\partial \Xi$ введем поле $\nu=\left(\nu_{1}, \nu_{2}, \nu_{3}\right)^{\top}$, нормированное равенством $|\nu(x)|=1$ и удовлетворяющее условию Липшица, и предположим, что вектор $u \in H^{1}(\Xi)^{3}$ подчинен соотношениям

$$
\begin{aligned}
u(x) & =0, & & x \in \Gamma^{\circ}(h):=\Gamma_{h}^{1} \cup \cdots \cup \Gamma_{h}^{I} ; \\
\nu(x)^{\top} u(x) & =0, & & x \in \Gamma^{\bullet}(h):=\Gamma_{h}^{I+1} \cup \cdots \cup \Gamma_{h}^{J} .
\end{aligned}
$$

Здесь $I \leqslant J$ и крайние случаи $I=0$ и $I=J$ не исключаются из рассмотрения. Формула $\left(2.18_{1}\right)$ означает, что тело $\Xi$ защемлено по нескольким малым окрестностям, и если $\nu$ - нормаль к гладкой поверхности $\partial \Xi$, то с некоторой натяжкой формула $\left(2.18_{2}\right)$ выражает линеаризованные условия контакта без трения 
(настоящие условия Синьорини формулируются как односторонние связи; см. книги [34], [6], [35] и др.). Следующее утверждение проверяется рассмотрением аналогичного (2.14) функционала:

$$
F(u)=\sum_{j=1}^{I} \int_{\Gamma_{h}^{j}} d(x)^{\top} u(x) d s_{x}+\sum_{j=I+1}^{J} \int_{\Gamma_{h}^{j}} d(x)^{\top} \nu(x) \nu(x)^{\top} u(x) d s_{x} .
$$

Лемма 2.2. Неравенство Корна

$$
\left\|\nabla_{x} u ; L_{2}(\Xi)\right\|^{2} \leqslant c(h) \mathscr{E}(u ; \Xi)
$$

выполнено для любого поля $u \in H^{1}(\Xi)^{3}$, подчиненного условиям (2.18), в том и только том случае, если оказывается невырожденной $(6 \times 6)$-матрища $F(d)$ в формуле (2.19).

ЗАмЕчАниЕ 2.2. Поскольку после замены $u(x) \mapsto d(x)$ в правой части $(2.19)$ каждое слагаемое - неотрицательная симметрическая матрица размером $6 \times 6$, даже одного “полного" условия Дирихле $\left(2.18_{1}\right)$ на $\Gamma_{h}^{1}$ достаточно для невырожденности суммы матриц. Пусть $I=0$ и $\nu$ - нормаль к гладкой границе $\partial \Xi$. Если $\partial \Xi$ - поверхность вращения относительно оси $x_{3}$, то вектор поворота $\theta(x)=\left(-x_{2}, x_{1}, 0\right)^{\top}$ вокруг этой оси удовлетворяет условиям $\left(2.18_{2}\right)$, но нарушает неравенство (2.20). Для шара в качестве контрпримера годится любой из трех линейно независимых векторов поворота относительно центра шара.

Лемма 2.2 не дает информации о зависимости множителя $c(h)$ в неравенстве Корна (2.20) от параметра $h$. Сформулируем пару утверждений, которые можно получить как следствие результатов из $\S 3$ (см. далее замечание 3.1 ). Для этого понадобится геометрическое требование заполнения пространства $\mathbb{R}^{6}$ :

$\left.1^{\circ}\right) \mathbb{R}^{6}=\mathfrak{L}\left\{d\left(P^{j}\right)^{\top} a \mid a \in \mathbb{R}^{3}\right.$ npu $\left.j=1, \ldots, I, a=\nu\left(P^{j}\right) n p u j=I+1, \ldots, J\right\}$. $\mathrm{B}$ правую часть помещена линейная оболочка столбцов из $\mathbb{R}^{6}$, которые найдены по матрице (2.8), вычисленной в точке $P^{j}$, и направлениям $a$, "подкрепленным" условиями (2.18) (полное условие Дирихле подкрепляет любое направление). Нетрудно проверить (см., например, [22]), что в случае $J=I$ требование $1^{\circ}$ ) обеспечено условием треугольника

$2^{\circ}$ ) среди точек $P^{1}, \ldots, P^{J}$ имеются три, расположенные в вершинах невырожденного треугольника.

ТеОрема 2.3. 1) Если справедливо условие $\left.1^{\circ}\right)$, то постоянная $c(h)$ в неравенстве Корна (2.20) удовлетворяет оценке

$$
c(h) \leqslant C h^{-m}
$$

nрu $m=1$.

2) Если $I \geqslant 1$, то верна оценка (2.21) при $m=3$.

ПримеР 2.2. Пусть $b \in \mathbb{R}^{6} \backslash\{0\}$ и

$$
u(x)=d(x) b-\sum_{j=1}^{I} \chi\left(h^{-1}\left|x-P^{j}\right|\right) d(x) b+\sum_{j=I+1}^{J} \chi\left(h^{-1}\left|x-P^{j}\right|\right) \nu(x) \nu(x)^{\top} d(x) b,
$$


где $\chi \in C_{0}^{\infty}(\mathbb{R})$ - “эталонная" срезающая функция, $\chi(t)=1$ при $|t| \leqslant 1$ и $\chi(t)=0$ при $|t| \geqslant 2$ (срезка $\chi_{\infty}(t)$, использованная в примере 2.1 , равна разности $1-\chi(t)$ при $t \geqslant 0$ и нулю при $t<0)$. Поле $u$ удовлетворяет условиям защемления и контакта (2.18). Очевидно, что

$$
\left\|u ; L_{2}(\Xi)\right\| \geqslant c_{0}|b|, \quad\left\|\nabla_{x} u ; L_{2}(\Xi)\right\| \geqslant c_{1}\left|b^{\prime \prime}\right| .
$$

Кроме того, $D\left(\nabla_{x}\right) u=0$ вне объединения $2 h$-окрестностей точек $P^{1}, \ldots, P^{J}$, а значит, вычислив функционал (2.6) при учете неравенства Липшица для поля $\nu$, обнаруживаем, что

$$
\mathscr{E}(u ; \Xi) \leqslant c\left(h\left\{\sum_{j=1}^{I}\left|d\left(P^{j}\right) b\right|^{2}+\sum_{j=1+I}^{J}\left|\nu\left(P^{j}\right)^{\top} d\left(P^{j}\right) b\right|^{2}\right\}+h^{3}|b|^{2}\right) .
$$

Если условие $\left.1^{\circ}\right)$ соблюдено, то выражение из фигурных скобок в $(2.23)$ положительное, т. е. в условиях теоремы $2.3,1)$ оценка $(2.21)$ с показателем $m<1$ невозможна. При отсутствии свойства (2.21) найдется столбец $b \in \mathbb{R}^{6} \backslash\{0\}$, для которого названное выражение равно нулю, а значит, $m \geqslant 3$ в оценке (2.21).

Пример 2.3. 1) Пусть $I=0, J=6$ в краевых условиях (2.18) и $\Xi-$ куб $\left\{x:\left|x_{k}\right|<1, k=1,2,3\right\}$. Геометрическое требование $\left.1^{\circ}\right)$ выполнено, если $\nu$ - единичная внешняя нормаль и при некотором $t \in(0,1)$ точки $P^{1}, \ldots, P^{6}$ определены так:

$$
\begin{array}{lll}
P^{1}=(1, t, 0)^{\top}, & P^{2}=(0,1, t)^{\top}, & P^{3}=(t, 0,1)^{\top}, \\
P^{4}=(-1,-t, 0)^{\top}, & P^{5}=(0,-1,-t)^{\top}, & P^{6}=(-t, 0,-1)^{\top} .
\end{array}
$$

Тело $\Xi$ с векторами $\nu^{1}, \ldots, \nu^{6}$ изображено на рис. 1 . В случае $t=0$ (точки помещены в центры граней куба) условие $1^{\circ}$ ) нарушено, но условие $2^{\circ}$ ) соблюдено.

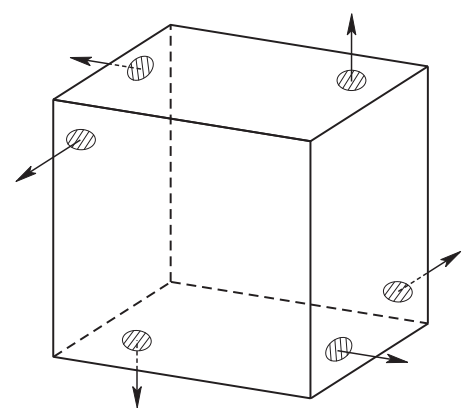

Рис. 1. Фиксация куба в шести малых зонах

2) Если $I=0$, но матрица $F(d)$ все-таки невырожденная, то точный показатель $m$ в оценке (2.21) может быть сколь угодно большим. Действительно, пусть $\Xi$ - цилиндр $\left\{x:\left|x^{\prime}\right|<1, H\left(x_{1}\right)<x_{3}<2\right\}$ с искривленным нижним основанием, причем $H\left(x_{1}\right)=x_{1}^{n}$ при $n \in \mathbb{N}$, а $\nu$ - единичная внешняя нормаль. Поскольку на днище нормаль задана формулами $\nu(x)=N\left(x_{1}\right)^{-1}\left(n x_{1}^{n-1}, 0,-1\right)^{\top}$ 
и $N\left(x_{1}\right)=\left(1+n^{2} x_{1}^{2 n-2}\right)^{1 / 2}$, вектор-функция $u(x)=\theta(x)-\nu(x) \mathbf{n}\left(x_{1}\right)^{-1} n x_{1}^{n-1} \times$ $x_{2} \chi\left(h^{-1}\left(x-P^{1}\right)\right)$ (ср. замечание 2.2$)$ удовлетворяет условиям $\left(2.18_{2}\right)$, если $I=0$, $P^{1}=(0,0, H(0))^{\top}$, а точки $P^{2}, \ldots, P^{J}$ произвольным образом разбросаны по боковой поверхности и верхнему основанию цилиндра. По-прежнему выполнены соотношения $(2.22)$, но теперь $m \geqslant 2 n+3$ в силу соотношения

$$
\mathscr{E}(u ; \Xi) \leqslant c n^{2} \int_{0}^{2 h}\left(h^{-2} r^{2 n+2}+r^{2 n}\right) r^{2} d r=C \frac{n^{2}(5 n+6)}{(n+1)(n+2)} h^{2 n+3} .
$$

В случае $I=0$ условие $1^{\circ}$ ) может быть выполнено лишь при $J \geqslant 6$. Таким образом, пяти пальцев на руке недостаточно для того, чтобы зафиксировать предмет, обеспечив наименьшую постоянную в неравенстве Корна. Впрочем, на Земле одно направление выделено гравитационным полем и с весомыми предметами человек обычно справляется одной рукой.

Зоны закрепления и контакта могут иметь произвольную форму, а множества $\Gamma_{h}^{j}=\partial \Xi \cap \mathbb{B}_{h}\left(P^{j}\right)$ обязаны лишь содержаться в них. В очередных утверждениях рассмотрим поля смещений, которые обращаются в нуль на $\mathscr{T}_{h}=$ $\partial \Xi \cap \mathscr{V}_{h}$, где $\mathscr{V}_{h}-R h$-окрестность гладкой дуги $\Upsilon \subset \partial \Xi$, замкнутой (тонкий обруч) или разомкнутой (узкая накладка).

ТеОрема 2.4. 1) Если дуга $\Upsilon$ не является прямолинейным отрезком, то поле $u \in \stackrel{\circ}{H^{1}}\left(\Xi ; \mathscr{T}_{h}\right)^{3}$ удовлетворяет неравенству Корна $(2.20)$, в котором

$$
c(h) \leqslant C(1+|\log h|)^{2} .
$$

2) Если $\Upsilon$ - отрезок прямой $\Lambda$ и $P^{1} \neq \Lambda\left(\right.$ или $\left.P^{1} \in \Lambda\right)$, то для $u \in$ $\stackrel{\circ}{H}^{1}\left(\Xi ; \mathscr{T}_{h} \cup \Gamma_{h}^{1}\right)^{3}$ выполнено неравенство Корна $(2.20)$ и множитель $c(h)$ подчинен оченке (2.25) (или (2.21) при $m=3$ ).

Отметим, что соотношение (2.25) выполнено лишь тогда, когда линейная оболочка $\mathfrak{L}\left\{d(x)^{\top} a \mid x \in \Upsilon \cup\left\{P^{1}\right\}, a \in \mathbb{R}^{3}\right\}$ (в условиях теоремы 2.4, 1) точка $P^{1}$ отсутствует) совпадает со всем пространством $\mathbb{R}^{6}$, т. е. для обеспечения наилучшей оценки нормы $\left\|u ; H^{1}(\Xi)\right\|$ требуется аналогичное $1^{\circ}$ ) условие. В случае $\Upsilon \cup\left\{P^{1}\right\} \subset \Lambda$ размерность подпространства $\mathfrak{L}(\ldots)$ равна пяти.

Возникновение множителя $1+|\log h|$ в формуле $(2.25)$ обусловлено неравенством

$$
\left\|\rho^{-1}(1+|\log \rho|)^{-1} u ; L_{2}(\Xi)\right\| \leqslant c\left\|u ; H^{1}(\Xi)\right\|,
$$

содержащим весовой множитель $\rho(x)=\operatorname{dist}(x, \Upsilon)$ и являющимся следствием такого варианта одномерного неравенстве Харди:

$$
\int_{0}^{1} \rho^{-1}|\log \rho|^{-2}|U(\rho)|^{2} d \rho \leqslant 4 \int_{0}^{1} \rho\left|\frac{d U}{d \rho}(\rho)\right|^{2} d \rho, \quad U \in C_{0}^{1}[0,1) .
$$

Подчеркнем, что соотношение (2.26) проверяется без учета краевого условия $u=0$ на множестве $\mathscr{T}_{h}$, а в случае замкнутой дуги $\Upsilon$ на гладкой поверхности $\partial \Xi$ получается в результате интегрирования неравенства (2.27) для функции $U=\chi u$ по длине дуги $s \in \Gamma$ и угловой переменной $\varphi \in(0, \pi)$. Говоря по-другому, весовой множитель с логарифмом, характерный для двумерных областей, обусловлен продолговатой формой зоны защемления $\mathscr{T}_{h}$. 
ЗАмечАниЕ 2.3. 1) Логарифмы. Пусть $\Xi=\left\{x: x_{1} \in(0,2),\left|x_{k}\right|<1\right.$, $k=2,3\}-$ куб, а $P^{1}=(2,0,0)$ и $\Upsilon=\left\{x: x_{1}=x_{2}=0,\left|x_{3}\right|<1\right\}$ - точка и отрезок на его противоположных гранях. Рассмотрим поле

$$
u(x)=\left(\chi_{\infty}\left(h^{-1}\left\|x-P^{1}\right\|\right)+\chi_{-}\left((\log h)^{-1} \log \left|x^{\prime}\right|\right)\right)\left\{x_{3} e_{1}-\left(x_{1}-2\right) e_{3}\right\},
$$

где $h<1, x^{\prime}=\left(x_{1}, x_{2}\right), e_{k}$ - орт оси $x_{k}, \chi_{\infty}$ - срезающая функция из примера 2.1 , а срезка $\chi_{-} \in C^{\infty}(\mathbb{R})$ определена так: $\chi_{-}(t)=1$ при $t<1 / 2$ и $\chi_{-}(t)=0$ при $t>1$. Ясно, что $\left\|u ; H^{1}(\Xi)\right\|^{2} \geqslant\left\|u ; L_{2}(\Xi)\right\|^{2} \geqslant c>0$. Поскольку поле из фигурных скобок в (2.28) является поворотом вокруг оси $\left\{x: x_{1}=2, x_{2}=0\right\}$, выкладка (2.23) показывает, что $\mathscr{E}\left(u ; \Xi_{-}\right) \leqslant c h^{3}$; здесь $\Xi_{ \pm}=\left\{x \in \Xi: \pm x_{1}> \pm 1\right\}$. Вычислим форму $\mathscr{E}\left(u ; \Xi_{+}\right)$на другой половина куба. Деформации $\varepsilon_{p q}(u)$ содержат производные срезки $\chi_{-}$, имеющей аргументом отношение логарифмов, а значит, носители компонент тензора деформаций расположены в полом цилиндре $\left\{x: h \leqslant r=\left|x^{\prime}\right| \leqslant h^{1 / 2}\right\}$, на котором $\left|\varepsilon_{p q}(u ; x)\right| \leqslant c|\log h|^{-1} r^{-1}$. Таким образом,

$$
\mathscr{E}\left(u ; \Xi_{+}\right) \leqslant C \int_{-1}^{1} d x_{3} \int_{h}^{h^{1 / 2}}|\log h|^{-2} r^{-2} r d r=\left.2 C|\log h|^{-2} \log r\right|_{h} ^{h^{1 / 2}}=C|\log h|^{-1} .
$$

Итак, неравенство (2.25) без множителя $1+|\log h|$ не может иметь места, однако автор не знает примера, который бы демонстрировал, что показатель 2 степени этого множителя неулучшаем ${ }^{4}$. Далее в тех случаях, когда неравенства Корна включают степень параметра $\log h$, вопрос о точности ее показателя обсуждаться не будет, так как ответ автору все равно неизвестен.

2) Если $\Upsilon$ - отрезок прямой $\Lambda$ и $P^{1} \in \Lambda$, то правильность оценки $(2.21)$ при $m=3$ устанавливается рассмотрением поля $u^{\theta}(x)=\left(1-\chi\left(h^{-1}\left|x^{\prime}\right|\right)\right) \theta(x)$, где $\theta-$ вектор поворота вокруг оси аппликат, и произвольных отрезка и точки на этой оси.

2.3. Шпаклевка каверн и шлифовка поверхности. Пусть символы $\Xi$ и $P^{1}, \ldots, P^{J}$ имеют тот же смысл, что и в предыдущем пункте. Пусть еще $\omega^{j}-$ область в $\mathbb{R}^{3}$ с компактным замыканием и $\omega_{h}^{j}=\left\{x: h^{-1}\left(x-P^{j}\right) \in \omega^{j}\right\}$ - ее сжатие в $h^{-1}$ раз при смещении центра. Предположим, что внутренность дополнения $\mathbb{R}^{3} \backslash \omega^{j}$ также является областью (например, $\omega^{j}$ - выпуклое множество), и рассмотрим тело с кавернами (малыми выемками) $\Xi(h)=\Xi \backslash\left(\overline{\omega_{h}^{1}} \cup \cdots \cup \overline{\omega_{h}^{J}}\right)$. Предполагаем, что при любом $h \in\left(0, h_{0}\right]$ граница $\partial \Xi(h)$ сильно липшицева с общей постоянной $M$ в условии Липшица. Если граница $\partial \Xi$ гладкая, достаточно потребовать, чтобы сильно липшицевыми были области $\mathbb{R}_{j-}^{3} \backslash \overline{\omega^{j}}$, где $\mathbb{R}_{j-}^{3}=\left\{x:-\nu\left(P^{j}\right)^{\top} x>0\right\}$, а $\nu\left(P^{j}\right)-$ внешняя нормаль в точке $P^{j} \in \partial \Xi$. Для шара $\omega^{j} \subset \mathbb{R}_{j-}^{3}$, касающегося границы полупространства, нужного свойства у области $\Xi(h)$ нет.

Введем область $\Omega^{j}$, обладающую свойствами области $\omega^{j}$ и содержащую замыкание $\overline{\omega^{j}}$. Обозначим $\Xi_{1 / h}^{j}$ область, получающуюся из $\Xi$ растяжением координат $x \mapsto y^{j}=h^{-1}\left(x-P^{j}\right)$, и внутри множества $G^{j}(h)=\left(\Xi_{1 / h}^{j} \cap \Omega^{j}\right) \backslash \omega^{j}$

\footnotetext{
${ }^{4}$ Согласно [7] в двумерном аналоге неравенства $\left(2.16_{1}\right)$ множитель $(\mathscr{R} / R)^{3}$ заменяется множителем $(\mathscr{R} / R)^{2} \log (\mathscr{R} / R)$. Вопрос о точности такого неравенства остается открытым (ср. статьи [7], [36]).
} 
выберем шар $\mathbb{B}_{R}^{j}$ фиксированного (не зависящего от $h$ ) радиуса $R$. Представим вектор-функцию $y^{j} \mapsto u^{j}\left(y^{j}\right)=u(x)$ в виде

$$
\begin{gathered}
u^{j}\left(y^{j}\right)=u^{j \perp}\left(y^{j}\right)+d\left(y^{j}\right) b^{j}, \\
\int_{\mathbb{B}_{R}^{j}} d\left(y^{j}\right)^{\top} u^{j \perp}\left(y^{j}\right) d y^{j}=0 \in \mathbb{R}^{6} .
\end{gathered}
$$

Для компоненты $u^{j \perp}$ верны неравенства Корна (предложение 2.1 и теорема 2.2)

$$
\begin{gathered}
\left\|u^{j \perp} ; H^{1}\left(\mathbb{B}_{R}^{j}\right)\right\|^{2} \leqslant c \mathscr{E}\left(u^{j \perp} ; \mathbb{B}_{R}^{j}\right)=c \mathscr{E}\left(u^{j} ; \mathbb{B}_{R}^{j}\right), \\
\left\|u^{j \perp} ; H^{1}\left(\Omega^{j}(h)\right)\right\|^{2} \leqslant c_{j}\left(\mathscr{E}\left(u^{j} ; \Omega^{j}(h)\right)+\left\|u^{j \perp} ; L_{2}\left(\mathbb{B}_{R}^{j}\right)\right\|^{2}\right),
\end{gathered}
$$

а столбец $b^{j} \in \mathbb{R}^{6}$ находится по формуле

$$
b^{j}=\left\{\int d\left(y^{j}\right)^{\top} d\left(y^{j}\right) d y^{j}\right\}^{-1} \int d\left(y^{j}\right)^{\top} u^{j}\left(y^{j}\right) d y^{j}
$$

(интегрируем по шару $\left.\mathbb{B}_{R}^{j}\right)$, где $\mathbf{d}\left(\mathbb{B}_{R}\right)$ - матрица Грама $\frac{4}{3} \pi R^{3} \operatorname{diag}\{1,1,1$, $\left.\frac{1}{5} R^{2}, \frac{1}{5} R^{2}, \frac{1}{5} R^{2}\right\}$ (ср. с матрицей $\left(2.15_{2}\right)$ ). В результате, используя продолжение $U^{j \perp} \in H^{1}\left(\Omega^{j} \cap \Xi_{1 / h}^{j}\right)^{3}$ поля $u^{j \perp}$, подчиненное оценке $\left\|U^{j \perp} ; H^{1}\left(\Omega^{j} \cap \Xi_{1 / h}^{j}\right)\right\|^{2} \leqslant$ $C_{j}\left\|u^{j \perp} ; H^{1}\left(\Omega^{j}(h)\right)\right\|^{2}$, положим

$$
U(x)= \begin{cases}u(x), & x \in \Xi(h), \\ U^{j \perp}\left(h^{-1}\left(x-P^{j}\right)\right)+d\left(h^{-1}\left(x-P^{j}\right)\right) b^{j}, & x \in \omega_{h}^{j} \cap \Xi,\end{cases}
$$

и заметим, что $D\left(\nabla_{x}\right) U=D\left(\nabla_{x}\right) U^{j \perp}$ на $\omega_{h}^{j} \cap \Xi$ и $\mathscr{E}(U ; \Xi) \leqslant c \mathscr{E}(u ; \Xi(h))$. Теперь, применяя предложение 2.1 , приходим к очередному утверждению.

ПРЕДЛОЖЕНИЕ 2.2. При выполнении указанных условий справедливо неравенство Корна

$$
\left\|\nabla_{x} u ; H^{1}(\Xi(h))\right\|^{2} \leqslant c\left(\mathscr{E}(u ; \Xi(h))+\left\|u ; L_{2}\left(\Xi^{\prime}\right)\right\|^{2}\right) .
$$

Предложение 2.2 сохраняет силу и для "тела с бугорками" $\Xi(h)=\Xi \cup$ $\omega_{h}^{1} \cup \cdots \cup \omega_{h}^{J}$ благодаря приведенному ниже следствию одномерного неравенства Харди.

Лемма 2.3. Если $\delta \in\left[0, \delta_{0}\right], \Xi^{\delta}=\left\{x \in \Xi:\left|x-P^{j}\right|>\delta, j=1, \ldots, J\right\}$ $u \quad \in \in H^{1}\left(\Xi^{\delta}\right)^{3}$, то выполнено неравенство

$$
\left\|\rho^{-1} u ; L_{2}\left(\Xi^{\delta}\right)\right\| \leqslant c\left\|u ; H^{1}\left(\Xi^{\delta}\right)\right\|,
$$

причем число $\delta_{0} \geqslant 0$ мало и $\rho(x)=\min \left\{\left|x-P^{j}\right| \mid j=1, \ldots, J\right\}$.

Предложение 2.1 для поля $u \in H^{1}(\Xi)^{3}$ и неравенство (2.32) позволяют оценить определенную формулой (2.17) норму $\mid\left\|u ; \mathbb{B}_{R h}^{j}\right\|_{h}$, где центр шара $\mathbb{B}_{R h}^{h}$ приближен к точке $P^{j}$ на расстояние $O(h)$. После растяжения координат $x \mapsto y^{j}$ можно применить неравенство $(2.17)$, в котором $\mathbb{B}_{R}=\mathbb{B}_{R}^{j}$, а область $\Xi$ заменена множеством $\omega^{j} \cup\left(\Omega^{j} \cap \Xi_{1 / h}^{j}\right)$, и распространить неравенство Корна на бугорки $\omega_{h}^{j} \backslash \Omega$. 
2.4. Неравенство Корна для тонкой пластины. Тонкая иилиндрическая пластина переменной толщины определяется равенством

$$
\Omega_{h}=\left\{x: x^{\prime} \in \omega,-h H_{-}\left(x^{\prime}\right)<x_{3}<h H_{+}\left(x^{\prime}\right)\right\},
$$

где $\omega$ - область на плоскости $\mathbb{R}^{2}$ с сильно липшицевой границей $\partial \omega, H^{ \pm}$- функции на множестве $\bar{\omega}$, подчиненные условию Липшица и соотношению $H\left(x^{\prime}\right)=$ $H_{+}\left(x^{\prime}\right)+H_{-}\left(x^{\prime}\right)>0, x \in \bar{\omega}$, a $h \in(0,1]$ - малый параметр (для того чтобы сделать его безразмерным, считаем, что $\operatorname{diam} \omega=1)$. Отметим, что при любом $h \leqslant 1$ область (2.33) является сильно липшицевой. Если $H=$ const $\left(H_{ \pm}=\right.$const), то тонкая область $\Omega_{h}$ называется пологой оболочкой (пластиной постоянной толщины или плитой). Поскольку для пластины одно направление выделено ее геометрической формой, удобно использовать обозначения

$$
u^{\prime}=\left(u_{1}, u_{2}\right), \quad y=\left(y_{1}, y_{2}\right), \quad y_{1}=x_{1}, \quad y_{2}=x_{2}, \quad z=x_{3} .
$$

По аналогии с формулой (2.14) определим функционал $F^{h}$ одним из равенств

$$
\begin{aligned}
& F^{h}(u)=h^{-1 / 2} \int_{\Omega_{h}} d^{h}(y, z)^{\top} u(y, z) d y d z \\
& F^{h}(u)=h^{-1 / 2} \int_{\Gamma_{h}} d^{h}(y, z)^{\top} u(y, z) d s_{y} d z
\end{aligned}
$$

При этом $\Gamma_{h}=\left\{(y, z): y \in \partial \omega,-h H_{-}(y)<z<h H_{+}(y)\right\}-$ боковая поверхность пластины и

$$
d^{h}(y, z)=\left(\begin{array}{cccccc}
1 & 0 & -\alpha y_{2} & \alpha h^{-1} z & 0 & 0 \\
0 & 1 & \alpha y_{1} & 0 & -\alpha h^{-1} z & 0 \\
0 & 0 & 0 & 0 & 0 & h
\end{array}\right)
$$

Матрица $d^{1}(y, z)$ имеет явное сходство с матрицей $(2.8)$ и получается из нее перестановкой столбцов и удалением из третьей строки переменных $y_{1}$ и $y_{2}$. Оба функционала $\left(2.35_{1}\right),\left(2.35_{2}\right)$ удовлетворяют требованиям $(2.11)$, так как порождают неособенные $(6 \times 6)$-матрицы Грама.

Доказательство следующего утверждения, заимствованное в существенном из статьи [8] (см. также [15; теорема 3.2.1]), столь просто и элегантно, что воспроизводится полностью.

ТеОрема 2.5. Если $h \in(0,1] u u \in H^{1}\left(\Omega_{h}\right)^{3}$, то справедливо неравенство Корна

$$
\mathbf{|} u ; \Omega_{h} \mathbf{\|}^{2} \leqslant c\left(\mathscr{E}\left(u ; \Omega_{h}\right)+\left|F^{h}(u)\right|^{2}\right),
$$

в котором анизотропная норма определена формулой

$$
\begin{aligned}
\left|u ; \Omega_{h}\right|=\{ & \int_{\Omega_{h}}\left[\sum_{i=1}^{2}\left(\sum_{k=1}^{2}\left|\frac{\partial u_{i}}{\partial y_{k}}\right|^{2}+h^{2}\left|\frac{\partial u_{i}}{\partial z}\right|^{2}+h^{2}\left|\frac{\partial u_{3}}{\partial y_{i}}\right|^{2}+\left|u_{i}\right|^{2}\right)\right. \\
& \left.\left.+\left|\partial_{z} u_{3}\right|^{2}+h^{2}\left|u_{3}\right|^{2}\right] d y d z\right\}^{1 / 2} .
\end{aligned}
$$


ДокаЗАТЕЛЬство. Замены $z \mapsto \zeta=h^{-1} z, u_{3} \mapsto w=h u_{3}$ обеспечивают соотношение $F^{h}(u)=h^{1 / 2} F^{1}\left(u^{\prime}, w\right)$. Кроме того,

$$
\begin{aligned}
\mathscr{E}\left(u ; \Omega_{h}^{0}\right)= & h \int_{\Omega_{1}}\left\{\sum_{i=1}^{2}\left(\left|\frac{\partial u_{i}}{\partial y_{i}}\right|^{2}+\frac{1}{2} h^{-2}\left|\frac{\partial u_{i}}{\partial \zeta}+\frac{\partial w}{\partial y_{i}}\right|^{2}\right)\right. \\
& \left.+\frac{1}{2}\left|\frac{\partial u_{1}}{\partial y_{2}}+\frac{\partial u_{2}}{\partial y_{1}}\right|^{2}+h^{-4}\left|\frac{\partial w}{\partial \zeta}\right|^{2}\right\} d y d \zeta \\
\geqslant & h \int_{\Omega_{1}}\left\{\sum_{i=1}^{2}\left(\left|\frac{\partial u_{i}}{\partial y_{i}}\right|^{2}+\frac{1}{2}\left|\frac{\partial u_{i}}{\partial \zeta}+\frac{\partial w}{\partial y_{i}}\right|^{2}\right)\right. \\
& \left.+\frac{1}{2}\left|\frac{\partial u_{1}}{\partial y_{2}}+\frac{\partial u_{2}}{\partial y_{1}}\right|^{2}+\left|\frac{\partial w}{\partial \zeta}\right|^{2}\right\} d y d \zeta \\
\geqslant & h c_{1}^{-1} \int \sum_{\Omega_{1}}^{2}\left(\sum_{i=1}^{2}\left|\frac{\partial u_{i}}{\partial y_{k}}\right|^{2}+\left|\frac{\partial u_{i}}{\partial \zeta}\right|^{2}+\left|\frac{\partial w}{\partial y_{i}}\right|^{2}+\left|u_{i}\right|^{2}\right) \\
& \left.+\left|\frac{\partial w}{\partial \zeta}\right|^{2}+|w|^{2}\right\} d y d \zeta-h\left|F^{1}\left(u^{\prime}, w\right)\right|^{2} .
\end{aligned}
$$

Последнее неравенство - результат применения предложения 2.1 в области $\Omega_{1}$, не зависящей от параметра $h$. Обратные замены превращают правую часть (2.39) в разность $c_{1}^{-1}\left|u ; \Omega_{h}\right|^{2}-\left|F^{h}(u)\right|^{2}$ за тем исключением, что фрагмент $\left|\partial_{z} u_{3}\right|^{2}$ подынтегрального выражения в формуле (2.38) приобретает лишний множитель $h^{4}$. Этот дефект устраняется легко, так как $\mathscr{E}\left(u ; \Omega_{h}\right) \geqslant$ $\left\|\partial_{z} u_{3} ; L_{2}\left(\Omega_{h}\right)\right\|^{2}$ благодаря определениям $(2.2),(2.4)$ и $(2.6)$.

Проверенное утверждение, разумеется, сохраняет силу и для пластин с уступами: в определении (2.33) функции $H_{ \pm}$могут быть кусочно липшицевыми.

ЗАмЕчАНИЕ 2.4. Еще один функционал, годящийся для правой части (2.37) (см. [12]), имеет вид

$$
F^{* h}(u)=h^{-1 / 2} \int_{\Omega_{h}^{*}} d^{h}(y, z)^{\top} u(y, z) d y d z,
$$

где $\Omega_{h}^{*}=\left\{x: y \in \omega^{*},-h H_{-}(y)<z<h H_{+}(y)\right\}$ и $\omega^{*}-$ непустая подобласть продольного сечения $\omega$ пластины $\Omega_{h}$. Кроме того, в согласии с формулой $(2.36)$ находим

$$
\left|F^{* h}(u)\right| \leqslant c\left(\left\|u^{\prime} ; L_{2}\left(\Omega_{h}^{*}\right)\right\|^{2}+h^{2}\left\|u_{3} ; L_{2}\left(\Omega_{h}^{*}\right)\right\|^{2}\right) .
$$

Если поле смещений $u$ удовлетворяет условию (2.13) на $\Gamma_{h}$, то функционал $\left(2.35_{2}\right)$ обращается в нуль и в правой части (2.37) остается только выражение $c \mathscr{E}\left(u ; \Omega_{h}\right)$. Как установлено в статье [9], полученное неравенство можно усилить за счет введения в норму (2.38) обратных степеней весового множителя $\rho_{h}(y)=h+\operatorname{dist}(y, \partial \omega)$, равного $O(h)$ в $\mathscr{R} h$-окрестности боковой поверхности пластины и $O(1)$ на удалении от нее (ср. с неравенством (1.5) в примере 1.3).

Теорема 2.6. Если $h \in(0,1] u u \in \stackrel{\circ}{H^{1}}\left(\Omega_{h} ; \Gamma_{h}\right)^{3}$, то справедливо неравенство Корна

$$
\mathbf{|} u ; \Omega_{h} \mathbf{|}_{0}^{2} \leqslant c \mathscr{E}\left(u ; \Omega_{h}\right),
$$


в котором весовая анизотропная норма определена формулой

$$
\begin{aligned}
\mathbf{|} u ; \Omega_{h}^{0} \mathbf{I}_{0}= & \left\{\int _ { \Omega _ { h } } \left[\sum_{i=1}^{2}\left(\sum_{k=1}^{2}\left|\frac{\partial u_{i}}{\partial y_{k}}\right|^{2}+h^{2} \rho_{h}^{-2}\left|\frac{\partial u_{i}}{\partial z}\right|^{2}+h^{2} \rho_{h}^{-2}\left|\frac{\partial u_{3}}{\partial y_{i}}\right|^{2}+\rho_{h}^{-2}\left|u_{i}\right|^{2}\right)\right.\right. \\
& \left.\left.+\left|\partial_{z} u_{3}\right|^{2}+h^{2} \rho_{h}^{-4}\left|u_{3}\right|^{2}\right] d y d z\right\}^{1 / 2}
\end{aligned}
$$

ПРимеР 2.4. Точность неравенств (2.37) и (2.41) проверяется при помощи следующего поля смещений, представляющего обычный асимптотический анзац в теории тонких пластин (см. статью [8] и, например, книги [37], [15], а также ср. с формулой $(2.10)$ в примере $2.1,1)$ ):

$$
u_{i}(y, z)=w_{i}(y)-h^{-1} z \partial_{i} w_{3}(y), \quad i=1,2, \quad u_{3}(y, z)=h^{-1} w_{3}(y)
$$

здесь $w_{p}$ - гладкие функции на множестве $\bar{\omega}, p=1,2,3$. В силу определения (2.36) матрицы $d^{h}(y, z)$ функционалы (2.35), вычисленные для поля $(2.43)$, суть $O(h)$. Деформации (2.2) приобретают порядок $1=h^{0}$, т. е. $\mathscr{E}\left(u ; \Omega_{h}\right)=$ $O(h)$. Кроме того, интеграл из фигурных скобок в (2.38) представим как сумма $I_{k}^{j}+I_{3}^{j}+I_{j}^{3}+I_{0}^{j}+I_{3}^{3}+I_{0}^{3}$, причем каждый из фрагментов, за исключением $I_{3}^{3}=0$, также оказывается равным $O(h)$. Наконец, назначив краевые условия $w_{p}=0, \nabla_{y} w_{3}=0$ на $\partial \omega$, обнаруживаем, что аналогичные интегралы $\mathbf{I}_{q}^{j}$ из фигурных скобок в (2.42) сохраняют порядок $h$, причем рост весовых множителей компенсируется вырождением полей на боковой поверхности $\Gamma_{h}$. Итак, весовые множители в нормах (2.38) и (2.42) расставлены правильно, а показатели степеней величин $h$ и $\rho_{h}^{-1}$ нельзя соответственно уменьшить и увеличить. Поскольку в столбце (2.4) производная $\partial_{z} u_{3}$ появляется один раз, рассчитывать на оценку какой-либо нормы, кроме $\left\|\partial_{z} u_{3} ; L_{2}\left(\Omega_{h}\right)\right\|=\left(I_{3}^{3}\right)^{1 / 2}$, не приходится.

ЗАмечАние 2.5. Строение нормы (2.42) показывает, что в $\mathscr{R} h$-окрестности $\mathscr{V}_{\mathscr{R} h}$ боковой поверхности $\Gamma_{h}$, на которой назначено условие Дирихле, имеет место оценка $\left\|\left|u ; \Omega_{h} \cap \mathscr{V}_{\mathscr{R} h} \|_{h} \leqslant c_{\mathscr{R}}\right| u ; \Omega_{h} \mathbf{l}_{0}\right.$ (см. формулу (2.17)). При помощи простого следствия неравенства Харди

$$
\frac{1}{h} \int_{\varphi}^{\varphi+h}|V(n)|^{2} d n \leqslant C \int_{\varphi}^{1}\left(\left|\frac{d V}{d n}(n)\right|^{2}+|V(n)|^{2}\right) d n,
$$

где $C$ не зависит от $\varphi \in[0,1 / 2], h \in(0,1 / 2)$ и $V \in H^{1}(\varphi, 1)$, получаем оценку

$\left\|\nabla_{y} u^{\prime}\right\|_{\diamond}+h\left\|\partial_{z} u^{\prime}\right\|_{\diamond}+h^{-1 / 2}\left\|u^{\prime}\right\|_{\diamond}+h\left\|\nabla_{y} u_{3}\right\|_{\diamond}+\left\|\partial_{z} u_{3}\right\|_{\diamond}+h^{1 / 2}\left\|u_{3}\right\|_{\diamond} \leqslant c \mid u ; \Omega_{h} \mathbf{|}$.

При этом | . . | - норма (2.42) (условий Дирихле на $\Gamma_{h}$ теперь нет) и для краткости норма в пространстве $L_{2}\left(\Omega_{h} \cap \mathscr{V}_{\mathscr{R} h}\right)$ обозначена $\|\cdot\|_{\diamond}$.

Условия (2.13) на $\Gamma_{h}$ означают, что жестко защемлена боковая поверхность пластины. Такой способ закрепления - чисто математическая абстракция и использовать его в реальных конструкциях глупо и опасно. Более подробное обсуждение моделей закрепления пластин можно найти в статье [38], а очередное утверждение, по сути доказанное в книге $[15 ; \S 3.3]$ и частично вытекающее из результатов работ [10], [39], относится к пластинам с достаточно произвольной формой кромки и зоны защемления. 
Пусть $\widetilde{\Omega}_{h}$ - область, совпадающая с пластиной $(2.33)$ внутри цилиндра $\omega \times \mathbb{R}$, причем ее кромка $G_{h}^{+}=\widetilde{\Omega}_{h} \backslash \overline{\Omega_{h}}$ принадлежит трубчатой окрестности $\mathscr{V}_{\mathscr{R} h}=$ $\{x: \operatorname{dist}(x, \partial \omega)<\mathscr{R} h\}$ контура ${ }^{5} \partial \omega \subset \mathbb{R}^{3}$. Через $G_{h}$ обозначаем расширенную кромку $\widetilde{\Omega}_{h} \cap \mathscr{V}_{2 \mathscr{R} h}$, включающую часть $G_{h}^{+}=G_{h} \cap \Omega_{h}$ цилиндрической пластины $\Omega_{h}$; кроме того, $G_{h}^{-}=G_{h} \backslash \overline{G_{h}^{+}}$. Пусть еще $\gamma \subset \partial \omega-$ непустая открытая дуга и $\widetilde{\Gamma}_{h}-$ непустое открытое множество на поверхности $\partial G_{h} \backslash \overline{\omega_{h}}$, лежащее внутри $2 \mathscr{R} h$-окрестности дуги $\gamma \subset \mathbb{R}^{3}$.

Предположим, что существует число $R>0$, при котором для любой точки $x^{0} \in \partial \omega$ выполнены следующие условия:

$3^{\circ}$ ) в пересечениях $G_{h}^{ \pm} \cap \mathbb{B}_{2 \mathscr{R} h}\left(x^{0}\right)$ найдутся шары $\mathscr{B}_{R h}^{ \pm}\left(x^{0}\right)$ радиусом $R h$, при которых справедливы аналогичные (2.17) неравенства

$$
\left\|u ; G_{h} \cap \mathbb{B}_{2 \mathscr{R} h}\left(x^{0}\right)\right\|_{h}^{2} \leqslant c\left(\mathscr{E}\left(u ; G_{h} \cap \mathbb{B}_{2 \mathscr{R} h}\left(x^{0}\right)\right)+h^{2}\left\|u ; L_{2}\left(\mathscr{B}_{R h}^{ \pm}\left(x^{0}\right)\right)\right\|^{2}\right),
$$

где постоянная с не зависит от $h \in\left(0, h_{0}\right], x^{0} \in \partial \omega u u \in H^{1}\left(G_{h}\right)^{3}$;

$\left.4^{\circ}\right)$ если $x^{0} \in \gamma$, то можно построить круговой цилиндр $\mathscr{C}_{2 \mathscr{R}}\left(x^{0}\right)$ высотой $2 \mathscr{R} h$, имеющий основанием большой круг шара $\mathscr{B}_{R h}^{+}\left(x^{0}\right)$ и пересекающий поверхность $\partial G_{h} \cap \mathbb{B}_{2 \mathscr{R} h}\left(x^{0}\right)$ внутри множества $\widetilde{\Gamma}_{h}$.

Обращаем внимание на различия в обозначениях: $\mathbb{B}_{\rho}\left(x^{0}\right)$ - шар с центром $x^{0}$, а у шара $\mathscr{B}_{R h}^{ \pm}\left(x^{0}\right)$ символ $x^{0}$ указывает только на принадлежность множеству $G_{h} \cap \mathbb{B}_{2 \mathscr{R} h}\left(x^{0}\right)$. Если это множество звездно относительно обоих шаров $\mathscr{B}_{R h}^{ \pm}\left(x^{0}\right)$, то неравенства (2.46) заведомо справедливо благодаря теореме 2.2.

Теорема 2.7. При выполнении сформулированных условий теорема 2.1 остается в силе для $u \in H^{1}\left(\widetilde{\Omega}_{h} ; \widetilde{\Gamma}_{h}\right)^{3}$ при замене цилиндрической пластины (2.33) пластиной $\widetilde{\Omega}_{h}$ с возмущенной кромкой. При этом норма (2.42) содержит весовой множитель $\rho_{h}(y)=h+\operatorname{dist}(y, \gamma)$.

2.5. Пластины с малыми дефектами или зонами защемления. Пусть $y^{0}$ - точка множества $\bar{\omega}=\omega \cup \partial \omega, \Xi \subset \mathbb{B}_{\mathscr{R}}(0)$ - область в пространстве $\mathbb{R}^{3}$ и $\Xi_{h}^{0}=\left\{x: h^{-1}\left(y-y^{0}, z\right) \in \Xi\right\}-$ ее сжатие в $h^{-1}$ раз. Под $h>0$ подразумевается тот же малый параметр, что и в формуле $(2.33)$ для пластины $\Omega_{h}$. Рассмотрим пластину $\Omega_{h}^{\bullet}=\Omega_{h} \cup \Xi_{h}^{0}$ с наростом и пластину $\Omega_{h}^{\circ}=\Omega_{h} \backslash \overline{\Xi_{h}^{0}}$ с каверной или отверстием. Как обычно, эти области считаем сильно липшицевыми. На части $\Gamma_{h}^{0}=\left\{x \in \Gamma_{h}: y \in \gamma\right\}$ боковой поверхности зададим условия Дирихле $(2.13)$; здесь $\gamma-$ непустая открытая дуга и если $y^{0} \in \bar{\gamma}$, то условия на $\partial \Xi_{h}^{0} \backslash \overline{\Omega_{h}}$ ставить не нужно.

Предположим, что найдутся такие положительные числа $R$ и $\mathscr{R}$, что

$\left.5^{\circ}\right)$ для некоторой пары шаров $\mathscr{B}_{R h}^{-} \subset \Xi_{h}^{0} \cap \Omega_{h}$ и $\mathscr{B}_{R h}^{+} \subset\left(\Omega_{h} \cap \mathbb{B}_{2 \mathscr{R} h}\left(x^{0}\right)\right) \backslash \Xi_{h}^{0}$ справедливы неравенства (2.46), в которых множество $G_{h}$ заменено пластиной $\Omega_{h}$.

Условие $5^{\circ}$ ) вполне аналогично условию $3^{\circ}$ ), введенному в п. 2.4. Оно также обеспечивает возможность применить процедуру заполнения каверн, изложенную в п. 2.3. Итак, стало понятно, как проверяется очередное утверждение.

\footnotetext{
5 Здесь и далее не различаем в обозначениях множества на плоскости $\mathbb{R}^{2}$ и их погружения в пространство $\mathbb{R}^{3}$ на плоскость $\{x: z=0\}$.
} 
СлЕДСТвИЕ 2.1. При указанных допущениях для поля и, принадлежащего пространству $\stackrel{\circ}{H}{ }^{1}\left(\Omega_{h}^{\bullet} ; \Gamma_{h}^{0} \backslash \overline{\Xi_{h}^{0}}\right)^{3}$ или $\stackrel{\circ}{H^{1}}\left(\Omega_{h}^{\circ} ; \Gamma_{h}^{0} \backslash \overline{\Xi_{h}^{0}}\right)^{3}$, выполнено весовое анизотропное неравенство Корна $(2.41)$, в котором роль весового множителя исполняет сумма $\rho_{h}(y)=h+\operatorname{dist}(y, \gamma)$, а пластина $\Omega_{h}$ заменена либо пластиной $\Omega_{h}^{\bullet}$, либо пластиной $\Omega^{\circ}$ с малыми дефектами.

Займемся теперь пластиной, защемленной по множествам с диаметрами $O(h)$. Условия (2.13) можно было бы ставить на поверхностях каверн или бугорков и постулировать аналогичные $3^{\circ}$ ) и $4^{\circ}$ ) геометрические свойства пластин с дефектами. Поскольку по изложенной в п. 2.4 схеме проверка неравенства Корна все равно сводится к анализу полей на цилиндрической пластине (2.33), ограничимся рассмотрением именно этого частного случая. Зафиксируем точки $P^{1}, \ldots, P^{J} \in \bar{\omega} \subset \mathbb{R}^{2}, P^{j} \neq P^{k}$ при $j \neq k$, и обозначим $\Gamma_{h}^{j}$ одно из двух множеств $\left\{x:\left|y-P^{j}\right|<R h, z= \pm H_{ \pm}(y)\right\}$, лежащих на верхнем и нижнем основаниях пластины.

ТЕОрема 2.8. Для поля $u \in \stackrel{\circ}{H^{1}}\left(\Omega_{h} ; \Gamma_{h}^{1} \cup \cdots \cup \Gamma_{h}^{J}\right)^{3}$ справедливо неравенство Корна

$$
\mathbf{\|} u ; \Omega_{h}^{0} \|_{\beta}^{2} \leqslant c \mathscr{E}\left(u ; \Omega_{h}\right),
$$

в котором анизотропная норма имеет вид

$$
\begin{aligned}
\mathbf{|}\left|u ; \Omega_{h}^{0}\right| \mathbf{I}_{\beta}=\{ & \int_{\Omega_{h}}\left[\sum_{i=1}^{2}\left(\left|\frac{\partial u_{i}}{\partial y_{i}}\right|^{2}+h^{2} \beta_{z}(h)\left(\left|\frac{\partial u_{i}}{\partial z}\right|^{2}+\left|\frac{\partial u_{3}}{\partial y_{i}}\right|^{2}\right)+\beta^{\prime}(h)\left|u_{i}\right|^{2}\right)\right. \\
& \left.\left.+\left|\frac{\partial u_{3}}{\partial z}\right|^{2}+\beta^{\prime}(h)\left(\left|\frac{\partial u_{1}}{\partial y_{2}}\right|^{2}+\left|\frac{\partial u_{2}}{\partial y_{1}}\right|^{2}\right)+h^{2} \beta_{z}(h)\left|u_{3}\right|^{2}\right] d y d z\right\}^{1 / 2},
\end{aligned}
$$

причем весовые множители определены так: $\beta_{z}(h)=(1+|\log h|)^{-2} u$

$$
\begin{array}{ll}
\beta^{\prime}(h)=(1+|\log h|)^{-2} & \text { в случае } J \geqslant 2, \\
\beta^{\prime}(h)=h^{2} & \text { в случае } J=1 .
\end{array}
$$

Правильность распределения весовых множителей в норме (2.48) подтверждена в примере $5.1,3)$. Теорема 4.4 в работе [40] не является точной, так как в ней затребовано условие треугольника $\left.2^{\circ}\right)$. В случае $\left(2.49_{1}\right)$ норма $(2.48)$ лишь логарифмическими множителями, медленно растущими при $h \rightarrow+0$, отличается от нормы (2.38). Иными словами, при игнорировании в норме (2.42) весовой функции $\rho_{h}$ защемление пластины по двум отстоящим одно от другого множествам диаметром $O(h)$ "почти равносильно" закреплению пластины по всей боковой поверхности. В данном случае впечатление о необходимости не менее трех точек опоры для устойчивости горизонтальной пластины ошибочно потому, что физическая картина ассоциируется с контактными условиями $\left(2.18_{2}\right)$, а не условиями $\left(2.18_{1}\right)$ защемления.

2.6. Неравенство Корна для тонкого стержня. Пусть $\Upsilon=(-1,1)-$ интервал и $\omega$ - область на плоскости $\mathbb{R}^{2}$, ограниченная гладким ${ }^{6}$ контуром $\partial \omega$. Пусть еще $\left\{\varkappa_{z}\right\}$ - семейство диффеоморфизмов: $\mathbb{R}^{2} \rightarrow \mathbb{R}^{2}$, гладко зависящих

\footnotetext{
${ }^{6}$ Термин "гладкий" здесь и далее означает “класса $C^{1}$ ”, хотя в большинстве ситуаций допустимы липшицевы границы.
} 
от параметра $z \in \bar{\Upsilon}$. Под регулярным слабоизогнутым стержнем с переменным сечением понимаем область

$$
G_{h}=\left\{x=(y, z) \in \mathbb{R}^{3}:|z|<1, \eta:=h^{-1} y \in \varkappa_{z} \omega\right\} .
$$

Торцы стержня (2.50) обозначаем $\Gamma_{h}^{ \pm}=\left\{x: z= \pm 1, \eta \in \varkappa_{ \pm 1} \omega\right\}$.

Следующее утверждение доказано в [10] (см. также [15; теорема 3.4.6]).

Теорема 2.9. Если $h \in(0,1] u u \in \stackrel{\circ}{H^{1}}\left(G_{h} ; \Gamma_{h}^{+}\right)$, то справедливо весовое неравенство Корна

$$
\mathbf{\|} u ; G_{h} \boldsymbol{|}_{+}^{2} \leqslant c \mathscr{E}\left(u ; G_{h}\right),
$$

в котором норма с весовым множителем $\rho_{h}(z)=h+|z-1|$ имеет вид

$$
\begin{aligned}
\mid u ; G_{h} \mathbf{|}_{+}= & \left\{\int _ { G _ { h } } \left[\sum_{i=1}^{2}\left(\left|\frac{\partial u_{i}}{\partial y_{i}}\right|^{2}+h^{2} \rho_{h}^{-2}\left(\left|\frac{\partial u_{i}}{\partial z}\right|^{2}+\left|\frac{\partial u_{3}}{\partial y_{i}}\right|^{2}\right)+h^{2} \rho_{h}^{-4}\left|u_{i}\right|^{2}\right)\right.\right. \\
& \left.\left.+h^{2} \rho_{h}^{-2}\left(\left|\frac{\partial u_{1}}{\partial y_{2}}\right|^{2}+\left|\frac{\partial u_{2}}{\partial y_{1}}\right|^{2}\right)+\left|\frac{\partial u_{3}}{\partial z}\right|^{2}+\rho_{h}^{-2}\left|u_{3}\right|^{2}\right] d y d z\right\}^{1 / 2} .
\end{aligned}
$$

Обращаем внимание на то, что условие Дирихле (2.13) поставлено только на одном торце $\Gamma_{h}^{+}$стержня $G_{h}$, и, соответственно, $\rho_{h}(z)=O(h)$ в ch-окрестности этого торца, но $\rho_{h}(z)=O(1)$ на удалении от него. По сравнению с пластиной (2.33) роль переменных (2.34) поменялась для стержня (2.50): теперь малыми оказываются размеры тонкого тела $G_{h}$ в направлении осей $y_{1}$ и $y_{2}$, а протяженность вдоль оси $z$ имеет единичный порядок. В частности, в норме (2.52) видоизменены множители при смещениях $u_{i}$ и $u_{3}$. В обеих нормах $(2.52)$ и $(2.42)$ продольные смещения, т. е. $u_{3}$ для стержня или $u^{\prime}$ для пластины, нагружены весом $\rho_{h}^{-1}$, а поперечные, т. е. соответственно $u^{\prime}$ или $u_{3},-$ весом $h \rho_{h}^{-1}$. Поскольку квадратичная форма (2.6) представляет собой эрзац упругой энергии, размещение весовых множителей отражает житейски очевидный факт: тонкое тело легче изогнуть, чем растянуть.

ЗАмЕчАнИЕ 2.6. Повторение способа [8] проверки неравенства Корна, представленное в доказательстве теоремы 2.5, не приводит к анизотропной норме $\left|u ; G_{h}\right|$, полученной удалением из $(2.52)$ коэффициентов $\rho_{h}^{-2}$ и $\rho_{h}^{-4}$ : нетрудно убедиться в том, что замены $y \mapsto \eta=h^{-1} y$ и $u^{\prime} \mapsto v=h u^{\prime}$ вместо слагаемого $h^{2}\left(\left|\partial_{2} u_{1}\right|^{2}+\left|\partial_{1} u_{2}\right|^{2}\right)$ в подынтегральном выражении (2.52) индуцируют слагаемое $h^{4}\left(\left|\partial_{2} u_{1}\right|^{2}+\left|\partial_{1} u_{2}\right|^{2}\right)$. На это обстоятельство было обращено внимание в статье [9], где было высказано предположение о возможности понизить степень параметра $h$. Само доказательство, опирающееся на лемму 1.1 работы [19], приведено в [10].

Пример 2.5. Главные члены асимптотического анзаца (см. [10], [13], [15; гл. 5] и др., а также ср. пример $2.1,3)$ ), принятого в теории тонких стержней, выглядят так:

$$
\begin{aligned}
& u_{1}(x)=h^{-1} w_{1}(z)-h^{-1} y_{2} w_{4}(z), \\
& u_{2}(x)=h^{-1} w_{2}(z)+h^{-1} y_{1} w_{4}(z), \\
& u_{3}(x)=w_{3}(z)-h^{-1}\left(y_{1} \partial_{z} w_{1}(z)+y_{2} \partial_{z} w_{2}(z)\right) .
\end{aligned}
$$


Если функции $w_{q} \in C^{\infty}[-1,1], q=1, \ldots, 4$, подчинить условиям $w_{q}(1)=0$, $\partial_{z} w_{1}(1)=\partial_{z} w_{2}(1)=0$, то поле $u$ с компонентами $\left(2.53_{1}\right)-\left(2.53_{3}\right)$ обращается в нуль на торце $\Gamma_{h}^{+}$. Поскольку

$$
\begin{aligned}
\varepsilon_{11}(u) & =\varepsilon_{22}(u)=\varepsilon_{12}(u)=0, & & \varepsilon_{33}(u ; x)=\partial_{z} w_{3}(z)-h^{-1} \sum_{i=1}^{2} y_{i} \partial_{z}^{2} w_{i}(z), \\
\varepsilon_{13}(u ; x) & =-h^{-1} y_{2} \partial_{z} w_{4}(z), & & \varepsilon_{23}(u ; x)=h^{-1} y_{1} \partial_{z} w_{4}(z),
\end{aligned}
$$

справедливо соотношение $\mathscr{E}\left(u ; G_{h}\right)=O\left(\operatorname{mes}_{3} G_{h}\right)=O\left(h^{2}\right)$ при условии, что хотя бы одна из функций $w_{q}$ ненулевая. Разбив интеграл из $(2.52)$ на фрагменты, обнаруживаем, что каждый из них, за исключением интегралов от выражений $\left|\partial u_{i} / \partial y_{i}\right|^{2}$, равных нулю, имеет тот же порядок $h^{2}$, а рост весовых множителей при $z \rightarrow+1$ компенсируется за счет вырождения полей в этой точке. Вместе в тем, в случае $w_{1}=w_{2}=w_{3}=0$ и $w_{4} \neq 0$ порядок указанных фрагментов понижается до $h^{4}$.

Итак, неравенство Корна (2.51) в принципе является точным, однако весовая норма (2.52) не приспособлена к так называемой крутильной составляющей $\theta(\eta) w_{4}(z)$ анзаца $\left(2.53_{1}\right)-\left(2.53_{3}\right)$. Для правильной обработки этой составляющей в очередном утверждении (см. предложение 3.4.13 и следствие 5.1.7 в книге [15]) обрабатывается еще один интеграл, содержащий разности $u_{i}-\bar{u}_{i}$, имеющие нулевое среднее по сечениям $\omega_{h}(z)$ стержня $G_{h}$; здесь

$$
\bar{u}_{i}(z)=\left(\operatorname{mes}_{2} \omega_{h}(z)\right)^{-1} \int_{\omega_{h}(z)} u_{i}(y, z) d y, \quad i=1,2 .
$$

Теорема 2.10. Если $h \in(0,1] u u \in H^{1}\left(G_{h}\right)^{3}$, то справедливо неравенство

$$
\left\|\partial_{z}\left(u_{i}-\overline{u_{i}}\right) ; L_{2}\left(G_{h}\right)\right\|^{2}+\left\|u_{i}-\overline{u_{i}} ; L_{2}\left(G_{h}\right)\right\|^{2} \leqslant c\left(\mathscr{E}\left(u ; G_{h}\right)+\left|u ; G_{h}\right|\right),
$$

причем |... - норма (2.52) при $\rho_{h}=1$.

Пусть $G_{h}^{ \pm}=\left\{x \in G_{h}: \pm z \in(1-h, 1)\right\}$ - кончики стержня $G_{h}$ с размерами $O(h)$ по всем трем направлениям. Благодаря распределению весовых множителей в норме $(2.52)$ находим, что $\left\|u ; G_{h}^{+}\left|\|_{h}^{2} \leqslant c\right| u ;\left.G_{h}^{+}\right|_{+} ^{2} \leqslant c\left|u ; G_{h}\right|_{+}^{2}\right.$. Приведем оценки норм смещений на другом кончике стержня $G_{h}^{-}$; при учете структуры нормы (2.52) они легко выводятся из соотношений (2.51) и (2.54) ввиду варианта (2.44) одномерного неравенства Харди.

СлЕДСтвиЕ 2.2. В условиях теоремы 2.10 имеет место формула

$$
\begin{aligned}
& \left\|u_{3} ; L_{2}\left(G_{h}^{-}\right)\right\|^{2}+\sum_{i=1}^{2}\left(h^{2}\left\|u_{i} ; L_{2}\left(G_{h}^{-}\right)\right\|^{2}+\left\|u_{i}-\overline{u_{i}} ; L_{2}\left(G_{h}^{-}\right)\right\|^{2}\right) \\
& \leqslant c\left(\mathscr{E}\left(u ; G_{h}\right)+\left|u ; G_{h}\right|^{2}\right) .
\end{aligned}
$$

Обсудим теперь нерегулярные тонкие стержни (в п. 2.8 это понятие будет расширено). Пусть сильно липшицева область $\widetilde{G}_{h}$ принадлежит $\mathscr{R} h$-окрестности множества (2.50) и содержит его. K форме тела не предъявляется никаких требований, кроме следующего: 
$\left.6^{\circ}\right)$ для любой точки $x^{0}=\left(0,0, z^{0}\right)^{\top}$ найдется такой шар $\mathscr{B}_{R h}\left(z^{0}\right) \subset G_{h} \cap$ $\mathbb{B}_{2 \mathscr{R} h}\left(x^{0}\right)$, что выполнено неравенство (2.46), в котором сделаны замены $G_{h} \mapsto \widetilde{G}_{h}$ и $\mathscr{B}_{R h}^{ \pm}\left(x^{0}\right) \mapsto \mathscr{B}_{R h}\left(z^{0}\right)$, а постоянная с и величина $R$ не зависят от параметра $h \in\left(0, h_{0}\right]$, точки $z^{0} \in \bar{\Upsilon}$ и поля $u \in H^{1}\left(\widetilde{G}_{h}\right)^{3}$.

Относительно части поверхности стержня $\widetilde{\Gamma}_{h}^{+} \subset \partial \widetilde{G}_{h} \cap \mathbb{B}_{2 \mathscr{R} h}(0,0,1)$, на которой назначено условие (2.13), предположим, что

$\left.7^{\circ}\right)$ существует круговой цилиндр $\mathscr{C}_{2 \mathscr{R} h}^{+}$высотой $2 \mathscr{R} h$, имеющий основанием большой круг шара $\mathscr{B}_{R h}(0,0,1)$ и пересекающий поверхность $\partial \widetilde{G}_{h} \cap$ $\mathbb{B}_{2 \mathscr{R h}}(0,0,1)$ внутри множества $\widetilde{\Gamma}_{h}^{+}$.

Отметим, что сформулированные требования полностью аналогичны условиям $\left.3^{\circ}\right)$ и $\left.4^{\circ}\right)$ из п. 2.4 и что центр шара $\mathbb{B}_{2 \mathscr{R} h}\left(x^{0}\right)$ помещен в точку $x^{0}$, но центр шара $\mathscr{B}_{R h}\left(z^{0}\right)$ не зафиксирован. В силу теоремы 2.2 неравенство (2.46) имеет место в том случае, когда при всех $z \in \bar{\Upsilon}$ область $\widetilde{G}_{h} \cap \mathbb{B}_{2 \mathscr{R} h}(0,0, z)$ звездна относительна шара $\mathscr{B}_{R h}(z)$.

Перенос неравенства Корна с регулярного стержня $G_{h}$ на нерегулярный стержень $\widetilde{G}_{h}$ осуществляется по схеме, предложенной в статье [10] (см. также [15; п. 3.4.1]).

Теорема 2.11. Пусть $h \in\left(0, h_{0}\right]$ и соблюдень требования $\left.6^{\circ}\right)$ u $\left.7^{\circ}\right)$. Тогда для поля $u \in \stackrel{\circ}{H}^{1}\left(\widetilde{G}_{h} ; \widetilde{\Gamma}_{h}\right)^{3}$ на нерегулярном тонком стержне $\widetilde{G}_{h} \supset G_{h}$ справедливо неравенство $(2.51)$, где вместо $G_{h}$ указана область $\widetilde{G}_{h}$, а норма $\left|u ; \widetilde{G}_{h}\right|_{+}$ вычислена по формуле (2.52).

2.7. Составные тела с контрастными жесткостями. Пусть тела $\Xi$ и $\Xi^{+} \subset \Xi$ имеют сильно липшицевы поверхности, а $\Xi^{-}=\Xi \backslash \bar{\Xi}^{+}$и $\Upsilon=$ $\partial \Xi^{+} \cap \partial \Xi^{-}-$непустые множества. Пусть $\mu>0$ - большой или малый физический параметр, характеризующий относительную жесткость тел. Рассмотрим аналогичную (2.6) квадратичную форму

$$
\mathscr{E}_{\mu}(u ; \Xi)=\left\|D\left(\nabla_{x}\right) u ; L_{2}\left(\Xi^{+}\right)\right\|^{2}+\mu\left\|D\left(\nabla_{x}\right) u ; L_{2}\left(\Xi^{-}\right)\right\|^{2} .
$$

Применяя неравенства Корна $(2.9)$ и (2.12) (в последнем функционал $F$ задан формулой (2.14), где граница Г заменена областью $\left.\Xi^{+}\right)$, находим, что

$$
\begin{aligned}
& \left\|u ; H^{1}\left(\Xi^{+}\right)\right\|^{2} \leqslant c\left(\mathscr{E}\left(u ; \Xi^{+}\right)+\left\|u ; L_{2}\left(\Xi^{+}\right)\right\|^{2}\right), \\
& \left\|u ; H^{1}\left(\Xi^{-}\right)\right\|^{2} \leqslant c\left\|u ; H^{1}(\Xi)\right\|^{2} \leqslant c\left(\mathscr{E}(u ; \Xi)+\left\|u ; L_{2}\left(\Xi^{+}\right)\right\|^{2}\right) .
\end{aligned}
$$

Умножив второе неравенство на $\min \{1, \mu\}$ и сложив с первым, после простых преобразований приходим к следующему утверждению.

ПРЕДЛОЖЕНИЕ 2.3. Для поля $u \in H^{1}(\Xi)^{3}$ верна оценка

$$
\left\|u ; H^{1}\left(\Xi^{+}\right)\right\|^{2}+\min \{1, \mu\}\left\|u ; H^{1}\left(\Xi^{-}\right)\right\|^{2} \leqslant c\left(\mathscr{E}_{\mu}(u ; \Xi)+\left\|u ; L_{2}\left(\Xi^{+}\right)\right\|^{2}\right) .
$$

Разумеется, норму $\left\|u ; L_{2}\left(\Xi^{+}\right)\right\|$в правой части (2.57) можно заменить любым подходящим функционалом, локализованным на множестве $\overline{\Xi^{+}}$, или убрать вообще, если часть $Г$ поверхности $\partial \Xi^{+} \backslash \bar{\Upsilon}$, имеющая положительную меру $\operatorname{mes}_{2} \Gamma$, жестко защемлена. 
ЗАмЕЧАниЕ 2.7. Если граница $\partial \Xi^{-}$также сильно липшицева (этого может не быть - см. пример 2.1), то, представив на множестве $\Xi^{-}$поле $u$ в аналогичном (2.29) виде

$$
\begin{gathered}
u(x)=u^{\perp}(x)+d(x) b, \\
\int_{\Gamma} d(x)^{\top} u(x) d s_{x}=0 \in \mathbb{R}^{6},
\end{gathered}
$$

находим при учете предложения 2.1, что

$$
\left\|u^{\perp} ; H^{1}\left(\Xi^{-}\right)\right\|^{2} \leqslant c \mathscr{E}\left(u^{\perp} ; \Xi^{-}\right)=c \mathscr{E}\left(u ; \Xi^{-}\right) .
$$

Матрица Грама $\left(2.15_{2}\right)$ невырожденная и поэтому выполнены неравенства

$$
|b| \leqslant c\left\|u ; L_{2}(\Gamma)\right\| \leqslant c\left\|u ; H^{1}\left(\Xi^{+}\right)\right\| .
$$

Итак, в случае $\mu>1$ неравенство (2.57) можно уточнить:

$$
\begin{aligned}
& \left\|u ; H^{1}\left(\Xi^{+}\right)\right\|^{2}+\mu\left\|u^{\perp} ; H^{1}\left(\Xi^{-}\right)\right\|^{2}+\min \{1, \mu\}\left\|d b ; H^{1}\left(\Xi^{-}\right)\right\|^{2} \\
& \quad \leqslant c\left(\mathscr{E}_{\mu}(u ; \Xi)+\left\|u ; L_{2}\left(\Xi^{+}\right)\right\|^{2}\right) .
\end{aligned}
$$

Иными словами, появление множителя $\min \{1, \mu\}$ в левой части (2.57) обусловлено лишь оценкой жесткого смещения $d(x) b$ в разложении $\left(2.58_{1}\right)$.

Очередные два утверждения о составных пластине и стержне вполне аналогичны предыдущему и приводятся без каких-либо комментариев. Пусть составная пластина $\Omega_{h}=\widetilde{\Omega}_{h}^{+} \cup \widetilde{\Upsilon} \cup \widetilde{\Omega}_{h}^{-}$и ее часть $\widetilde{\Omega}_{h}$ удовлетворяют условиям теоремы 2.7 .

ПреДЛОЖЕНИЕ 2.4. При указанных ограничениях справедливо неравенство Корна

$$
\mathbf{|} u ; \widetilde{\Omega}_{h}^{+} \mathbf{|}^{2}+\min \{1, \mu\} \| u ; \widetilde{\Omega}_{h}^{-} \mathbf{|}^{2} \leqslant c\left(\mathscr{E}_{\mu}\left(u ; \Omega_{h}\right)+\left\|u^{\prime} ; L_{2}\left(\widetilde{\Omega}_{h}^{+}\right)\right\|^{2}+h^{2}\left\|u_{3} ; L_{2}\left(\widetilde{\Omega}_{h}^{+}\right)\right\|^{2}\right),
$$

в котором форма $\mathscr{E}_{\mu}\left(u ; \Omega_{h}\right)$ определена аналогично $(2.56)$, а норма | . . | имеет вид (2.38).

Последнюю пару слагаемых в правой части (2.59) можно заменить выражением $|F(u)|^{2}$, где, например, $F-$ функционал $(2.40)$ при $\overline{\Omega_{h}^{*}} \subset \Omega_{h}^{+}$.

Пусть теперь $G_{h}$ - регулярный стержень $(2.50)$ и $\widetilde{G}_{h}^{ \pm}-$две его части, разделенные поверхностью $\widetilde{\Upsilon}_{h}$ диаметром $O(h)$, причем стержень $\widetilde{G}_{h}^{+}$защемлен по множеству $\Gamma_{h}^{+}$, но может быть нерегулярным (см. условия $6^{\circ}$ ) и $7^{\circ}$ ) в п. 2.6). Следующее утверждение, вытекающее из теорем 2.10 и 2.11, пересказывает теорему 4.4 работы [40].

ПРЕДЛОЖЕНИЕ 2.5. При указанных ограничениях поле $u \in \stackrel{\circ}{H^{1}}\left(G_{h} ; \Gamma_{h}^{+}\right)^{3}$ удовлетворяет неравенству Корна $\mid u ; \widetilde{G}_{h}^{+} \mathbf{|}_{+}^{2}+\min \{1, \mu\} \mathbf{|} u ; \widetilde{G}_{h}^{-} \mathbf{|}^{2} \leqslant c \mathscr{E}_{\mu}\left(u ; G_{h}\right)$, где норма |... | определена формулой $(2.52)$, а норма |...| - той же формулой при $\rho_{h}=1$.

Предложение 2.5 не исключает случай малого, размера $O(h)$, множества $\widetilde{G}_{h}^{-}$. 
2.8. Периодические стержни и пластины. Пусть $S$ - область, расположенная в слое $\left\{x: x_{3} \in(0,1)\right\}$, а $S_{h}=\left\{x: h^{-1} x \in S\right\}$ - ее сжатие в $h^{-1}=N$ раз; здесь $N \in \mathbb{N}$ - большое натуральное число. При целом $j \in \mathbb{Z}$ обозначим $\mathscr{S}(j)$ и $\mathscr{S}_{h}(j)$ сдвиги указанных множеств соответственно на расстояния $j$ и $h j$ вдоль оси аппликат. Предположим, что внутренность $G_{h}$ объединения $\bar{G}_{h}=\overline{\mathscr{S}_{h}(-N)} \cup \cdots \cup \overline{\mathscr{S}_{h}(N-1)}$ оказывается сильно липшицевой областью (в частности, связным множеством). Эту область называем прямым стержнем с периодически изменяющимся сечением или просто периодическим стержнем.

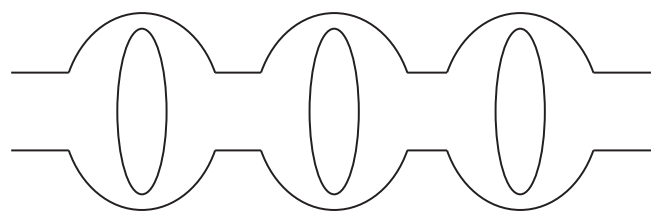

Рис. 2. Ёлочные бусы - сечение периодического стержня

Если стержень $G_{h}$ содержит круговой цилиндр $\{x:|y|<R h,|z|<1\}$, то для проверки теоремы 2.1 в случае периодического стержня годится разработанный в [10] (см. также [15; § 3.4]) прием распространения весового неравенства Корна с узкого множества на более широкое. "Елочные бусы" (их сечение изображено на рис. 2) не обладают названным свойством, однако для них и вообще для любых периодических стержней нужный цилиндр можно построить искусственно. Опишем соответствующую конструкцию.

Рассмотрим поле $u \in H^{1}(\mathfrak{S}(N))^{3}$ на квазицилиндре $\mathfrak{S}(N)$ (внутренность объединения множеств $\overline{S(j)}$, где $j \in \mathbb{Z}$ и $-N \leqslant j \leqslant N-1$ ), а затем перейдем к периодическому тонкому стержню $G_{h}$ при помощи сжатия координат. Сначала выберем какую-либо "шайбу" $\sigma=\left\{x:\left|y-y^{0}\right|<\mathscr{R},\left|z-z^{0}\right|<\mathscr{L}\right\} \subset \mathscr{S}$ и ее сдвиги $\sigma(j), j \in \mathbb{Z}$, вдоль оси аппликат. От шайбы $\sigma(0)$ к шайбе $\sigma(1)$ можно протянуть "рукав" $\tau \subset\left\{x: z^{0} \leqslant z \leqslant 1-z^{0}\right\}$ внутри объединения $\overline{S(0)} \cup \overline{S(1)}$ двух соседних ячеек, а также "штырь" $\pi=\left\{x:\left|y-y^{0}\right|<\mathscr{R}, z \in\left[z^{0}, 1-z^{0}\right]\right\}$, часть которого может заходить за пределы упомянутого объединения. Воспользуемся представлением $\left(2.29_{1}\right)$ на шаре $\mathbb{B}_{R}^{j} \subset \sigma(j)$ с центром $\left(y^{0}, z^{0}+j\right)$ и радиусом $R=\min \{\mathscr{R}, \mathscr{L}\}$ и распространим это представление на множество $T_{j}=$ $\sigma(j) \cup \tau(j) \cup \sigma(j+1)$. Имеется оператор продолжения $\gamma_{0}: H^{1}\left(T_{0}\right) \rightarrow H^{1}\left(T_{0} \cup \Pi_{0}\right)$, где $\Pi_{j}$ - круговой цилиндр $\sigma(j) \cup \pi(j) \cup \sigma(j+1)$. Композицию операторов продолжения и сдвига обозначаем $\gamma_{j}: H^{1}\left(T_{j}\right) \rightarrow H^{1}\left(\Pi_{j}\right)$. На множестве $T_{j}$ положим $\hat{u}^{j}=\gamma_{j} u^{j \perp}+d b^{j}$. Заметим, что, во-первых, $\hat{u}^{j}=u$ на шайбах $\sigma(j)$ и $\sigma(j+1)$ и, во-вторых, согласно соотношениям $(2.30),(2.17)$ и замечанию 2.1 имеем

$$
\mathscr{E}\left(\hat{u}^{j} ; \Pi_{j}\right)=c \mathscr{E}\left(\gamma_{j} u_{\perp}^{j} ; \Pi_{j}\right) \leqslant c\left\|\gamma_{j} u_{\perp}^{j} ; H^{1}\left(\Pi_{j}\right)\right\|^{2} \leqslant c\left\|u_{\perp}^{j} ; H^{1}\left(T_{j}\right)\right\|^{2} \leqslant c \mathscr{E}\left(u ; T_{j}\right) .
$$

Благодаря первому из упомянутых свойств справедливо равенство $\hat{u}^{j}(x)=$ $\hat{u}^{j+1}(x)$ при $x \in \sigma(j+1)$, т. е. формулы $\hat{u}=\hat{u}^{j}$ на $\Pi_{j}$ определяют поле $\hat{u} \in$ $H^{1}(\mathfrak{C}(N))^{3}$ на круговом цилиндре $\mathfrak{C}(N)=\left\{x:\left|y-y^{0}\right|<R,|z|<N\right\}$. Кроме того, второе свойство обеспечивает неравенство $\mathscr{E}(\hat{u} ; \mathfrak{C}(N)) \leqslant c \mathscr{E}(u ; \mathfrak{S}(N))$, инвариантное относительно сжатия координат. 
Итак, схема проверки неравенства Корна для произвольного периодического стержня состоит из двух этапов. Сначала для поля смещений $u \in H^{1}\left(G_{h}\right)^{3}$ строится продолжение $\hat{u}$ на опорныц тонкий круговой стержень $\mathfrak{C}_{h}$ и оценивается подходящая весовая анизотропная норма $\left|\hat{u} ; \mathfrak{C}_{h}\right| \ldots$, квадрат которой заведомо больше суммы $\sum\left|u ; \sigma_{h}(y)\right|_{\ldots}^{2}$ (суммирование по $\left.j=-N, \ldots, N\right)$. Затем при помощи метода [10] (см. также [15; гл. 3] и п. 2.4) устанавливаются соотношения $\left|u ; \mathscr{S}_{h}(j)\right|_{\ldots}^{2} \leqslant c\left(\mathscr{E}\left(u ; \mathscr{S}_{h}(j)\right)+\left|u ; \sigma_{h}(j)\right|_{\ldots}^{2}\right)$, суммирование которых по $j=-N, \ldots, N-1$ приводит к нужному результату.

Здесь утверждение о неравенстве Корна для периодического тонкого стержня не формулируется - оно выглядит в точности так же, как теоремы 2.9, 2.11, и требует предположения $7^{\circ}$ ) из п. 2.6. Отметим, что приемы, разработанные в [15; гл. 3], позволяют приспособить весовое анизотропное неравенство Корна к локально периодическим слабоискривленным стержням. Помимо этого, результаты, относящиеся к периодическим стержням, составляют базу для существенного расширения понятия "нерегулярный тонкий стержень” (см. п. 2.6).

Более изощренная процедура потребовалась в работах [41], [14] для построения опорной - сплошной и цилиндрической - плиты в случае произвольной периодической пластины (ее толщина и периоды осцилляции границы сравнимы по порядку). Эта процедура напоминает компьютерную игру "tetris" и оперирует со вспомогательными объектами: крестовинами, брусками, коромыслами и плитками. В итоге весовые анизотропные неравенства Корна, подобные указанным теоремами 2.5-2.7, устанавливаются для локально периодических слабоискривленных пластин без каких-либо ограничений на геометрическое строение ячеек (подробности см. в статьях [41], [14]).

Требования $3^{\circ}$ ) и $4^{\circ}$ ) в п. 2.4 можно формулировать не для всех точек $x^{0}$ на кривых $\partial \omega$ и $\gamma$, но для конечного их набора $x^{1}, \ldots, x^{N(h)}$ при условии, что шары $\mathbb{B}_{2 \mathscr{R} h}\left(x^{1}\right), \ldots, \mathbb{B}_{2 \mathscr{R} h}\left(x^{N(h)}\right)$ покрывают кромку пластины (число $N(h)$ зависит от параметра $h$ ). В частности, теорема 2.7 сохраняет силу, если крепление пластины $\widetilde{\Omega}_{h}$ осуществляется по краю путем точечной пайки диаметром $O(h)$ на расстояниях $O(h)$. То же самое можно сказать и о теореме 2.4 при закреплении тела $\Xi$ вдоль дуги $\Upsilon$. Защемление часовой шестерни по концам зубцов доставляет еще один пример периодической области, которая исследуется при помощи изложенных выше приемов (см. [42] по поводу асимптотического анализа пластин с изрезанными краями). Количество подобных примеров можно значительно увеличить. В частности, сочленения тела и пластин, исследуемые в 4 , допускают крепление "на шипах" (мелкие, часто расположенные пазы) или при помощи уже упоминавшейся периодической точечной пайки. При этом сформулированные выше утверждения не претерпевают сколько-нибудь существенных изменений.

\section{§ 3. Неравенства Корна для сочленения массивного тела со стержнями}

3.1. Геометрия сочленения. На гладкой границе $\partial \Xi$ тела $\Xi$ выберем точки $P^{1}, \ldots, P^{J}$, из которых наружу выпустим лучи $\Lambda^{j}$ вдоль векторов $\nu^{j}, j=$ $1, \ldots, J$. Предположим, что $P^{j} \neq P^{k}$ при $j \neq k$ и векторы $\nu^{j}$ не являют- 
ся касательными к поверхности $\partial \Xi$ в точках $P^{j}$. Введем систему декартовых координат $\left(y^{j}, z^{j}\right)$ с центром $P^{j}$, совместив положительную полуось $z^{j}$ с лучом $\Lambda^{j}$. Пусть $\omega^{j}-$ ограниченная сильно липшицева область на плоскости и $G_{h}^{j}=\left\{x ; z^{j} \in\left(-h \lambda_{j}, l_{j}\right), \eta^{j}:=h^{-1} y^{j} \in \omega^{j}\right\}$ - цилиндрический прямой стержень, а $h>0$ - малый параметр. Масштабированием сделаем этот параметр безразмерным, а границу $h_{0}$ его изменяемости и величины $l_{j}>0$ и $\lambda_{j}>0$ зафиксируем так, чтобы стержни $G_{h}^{1}, \ldots, G_{h}^{J}$ взаимно не пересекались, а их торцы $\omega_{h}^{j}\left(-h \lambda_{j}\right)$ располагались внутри тела $\Xi ;$ здесь $\omega_{h}^{j}\left(z^{0}\right)=\left\{x: z=z^{0}\right.$, $\left.\eta^{j} \in \omega^{j}\right\}$ - сечение стержня. В дальнейшем система координат $x$ привязана к телу $\Xi$, а для стержней $G_{h}^{j}$ применяются системы $\left(y^{j}, z^{j}\right)$.

Под сочленением массивного тела с тонкими стержнями подразумевается множество

$$
\Omega(h)=\Xi \cup G_{h}^{1} \cup \cdots \cup G_{h}^{J},
$$

а $\Xi(h)=\Xi \backslash\left(\overline{G_{h}^{1}} \cup \cdots \cup \overline{G_{h}^{J}}\right)$ - само тело с гнездами $g_{h}^{i}$, в которые стержни и вставлены.

Материалы, из которых сделаны тело и стержни, считаем контрастными, т. е. рассматриваем такой эрзац упругой энергии (ср. с определениями (2.6) и $(2.56))$ :

$$
\mathscr{E}_{\mu}(u ; \Omega(h))=\left\|D\left(\nabla_{x}\right) u^{0} ; L_{2}(\Xi(h))\right\|^{2}+\mu \sum_{j=1}^{J}\left\|D\left(\nabla_{x}\right) u ; L_{2}\left(G_{h}^{j}\right)\right\|^{2} .
$$

Здесь $\mu>0$ - относительная (в сравнении с телом) жесткость стержней, а $u^{0}$ и $u^{j}$ - сужение поля $u \in H^{1}(\Omega(h))^{3}$ на подобласти $\Xi(h)$ и $G^{j}$.

Многие свойства геометрических объектов были затребованы лишь для упрощения изложения: стержни могут быть слабоискривленными, периодическими или нерегулярными, векторы $\nu^{1}, \ldots, \nu^{J}$ - касаться границы $\partial \Xi$, а сама она - быть только сильно липшицевой. Стержни не обязательно вставлять в пазы - допустимо приклеивание их к поверхности тела встык (при этом формула (3.2) нуждается в заменах $\left.\Xi(h) \mapsto \Xi и G_{h}^{j} \mapsto G^{j}(h)=G_{h}^{j} \backslash \bar{\Xi}\right)$. Кончикам стержней и гнездам можно придать достаточно произвольную форму. Во всех случаях требуются предположения, аналогичные предположениям $6^{\circ}$ ) и $7^{\circ}$ ) из п. 2.6, а также незначительные изменения в анализе, которые уже были разъяснены в $\S 2$. Поэтому рассматриваем только прямые цилиндрические стержни, что позволяет избежать повторений, устранить мелкие технические детали и высветить основные приемы доказательств.

3.2. Специальное расположение шести или более стержней. Предположим, что выполнено условие заполнения пространства $\mathbb{R}^{6}$, т. е., как и в п. 2.2 ,

$$
\left.1^{\circ}\right) \mathbb{R}^{6}=\mathfrak{L}\left\{d\left(P^{j}\right)^{\top} \nu^{j} \mid j=1, \ldots, J\right\} .
$$

ПримеР 3.1. 1) Морская мина. Если $P^{1}, \ldots, P^{J}$ - точки (2.24) на поверхности шара $\mathbb{B}_{R}(0)$ радиусом $R=\left(1+t^{2}\right)^{1 / 2}$, то условие $\left.1^{\circ}\right)$ соблюдено, например, в том случае, когда $t>0$ и векторы $\nu^{j}$ направлены вдоль нормалей к сфере $\partial \mathbb{B}_{R}(0)$ (рис. 3). При $t=0$ векторы $\nu^{j}$ попадают на оси декартовых координат и условие $1^{\circ}$ ) нарушается. 


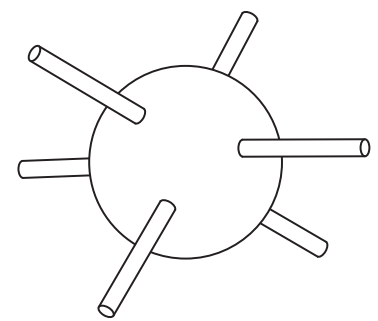

Рис. 3. Морская мина

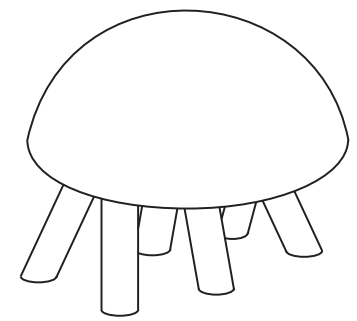

Рис. 4. Божья коровка

2) Божья коровка. Пусть $\Omega$ - полуэллипсоид $\left\{x: x_{1}^{2}+4 x_{2}^{2}+\frac{1}{4} x_{3}^{2}<9\right\}$, к которому снизу присоединены шесть тонких стержней $G_{h}^{1}, \ldots, G_{h}^{6}$ с такими атрибутами $\left(\nu^{j}\right)^{\top}$ и $\left(P^{j}\right)^{\top}$ :

$$
\begin{aligned}
& j=1: \quad\left(0,-2^{-1 / 2},-2^{-1 / 2}\right), \quad\left(-2,-2^{-1}, 0\right) ; \\
& j=2: \quad(0,0,-1), \quad(0,-1,0) \text {; } \\
& j=3: \quad\left(2^{-1},-2^{-1},-2^{-1 / 2}\right), \quad\left(2,-2^{-1}, 0\right) \text {; } \\
& j=4: \quad\left(2^{-1}, 2^{-1},-2^{-1 / 2}\right), \quad\left(2,2^{-1}, 0\right) \text {; } \\
& j=5: \quad(0,0,-1), \quad(0,1,0) \text {; } \\
& j=6: \quad\left(0,2^{-1 / 2},-2^{-1 / 2}\right), \quad\left(-2,2^{-1}, 0\right) .
\end{aligned}
$$

Сочленение изображено схематично на рис. 4. Громоздкие, но простые вычисления устанавливают справедливость равенства $1^{\circ}$ ).

Будем считать, что торцы $\Gamma_{h}^{j}=\omega_{h}^{j}\left(l_{j}\right)$ стержней, не погруженные в тело $\Xi$, жестко защемлены, т. е. примем условия $\left(2.18_{1}\right)$ при $j=1, \ldots, J$. Тогда благодаря теореме 2.9 и следствию 2.2 , примененным для стержня $G_{h}^{j}$, имеем

$$
h^{-1} \mu\left\|\left(\nu^{j}\right)^{\top} u^{j} ; L_{2}\left(g_{h}^{j}\right)\right\| \leqslant c \mu \mid u^{j} ; G_{h}^{j} \mathbf{I}_{j}^{2} \leqslant c \mu \mathscr{E}_{1}\left(u ; G_{h}^{j}\right) \leqslant c \mathscr{E}_{\mu}(u ; \Omega(h)) .
$$

Норма $\mid u^{j} ; G_{h}^{j} \mathbf{I}_{j}$ вычисляется по формуле $(2.52)$, в которой $G_{h}=G_{h}^{j}$ и $\rho_{h}\left(z^{j}\right)=$ $h+\left|z^{j}-l_{j}\right|$. Подчеркнем, что $\left(\nu^{j}\right)^{\top} u^{j}-$ проекция вектора смещений на ось стержня.

На множестве $\Xi$ поле $u$ представимо в виде $\left(2.58_{1}\right)$, причем в условии ортогональности $\left(2.58_{2}\right)$ интеграл по границе $\Gamma=\partial \Xi$ можно заменить любым подходящим функционалом $F$ из предложения 2.1. Составляющая $u^{\perp}$ разложения $\left(2.58_{1}\right)$ допускает оценку

$$
\begin{aligned}
\left\|u^{\perp} ; g_{h}^{j} \mid\right\|_{h}^{2} & \leqslant c\left(\left\|\nabla_{x} u^{\perp} ; L_{2}\left(g_{h}^{j}\right)\right\|^{2}+\left\|\rho^{-1} u^{\perp} ; L_{2}\left(g_{h}^{j}\right)\right\|^{2}\right) \\
& \leqslant c\left\|u^{\perp} ; H^{1}(\Xi)\right\|^{2} \leqslant c \mathscr{E}(u ; \Xi) \leqslant c \max \left\{1, \mu^{-1}\right\} \mathscr{E}_{\mu}(u ; \Xi) .
\end{aligned}
$$

Здесь использованы лемма 2.3 при $\delta=0$, предложение 2.1 и соотношение $D\left(\nabla_{x}\right) u^{\perp}=D\left(\nabla_{x}\right) u$.

Умножим равенство $\left(2.58_{1}\right)$ слева на матрицу $d(x)^{\top} \nu^{j}\left(\nu^{j}\right)^{\top} \in \mathbb{R}^{6 \times 3}$ и проинтегрируем по гнезду $g_{h}^{j}$. Сложив полученные интегралы, приходим к следующей системе шести алгебраических уравнений для столбца $b \in \mathbb{R}^{6}$ :

$$
M b=f .
$$


B' формуле (3.6) $M$ - матрица размером $6 \times 6, f \in \mathbb{R}^{6}-$ столбец,

$$
\begin{aligned}
M & =\sum_{j=1}^{J} M^{j}, & M^{j} & =\int_{g_{h}^{j}} d(x)^{\top} \nu^{j}\left(\nu^{j}\right)^{\top} d(x) d x, \\
f & =\sum_{j=1}^{J} f^{j}, & f^{j} & =\int_{g_{h}^{j}} d(x)^{\top} \nu^{j}\left(\nu^{j}\right)^{\top}\left[u(x)-u^{\perp}(x)\right] d x .
\end{aligned}
$$

Столбцы $\left(3.7_{2}\right)$ обрабатываем при помощи оценок (3.5) и (3.4). Именно,

$$
\begin{aligned}
\left|f^{j}\right|^{2} & \leqslant c \operatorname{mes}_{3} g_{h}^{j}\left(\int_{g_{h}^{j}}|u(x)|^{2} d x+\int_{g_{h}^{j}}\left|u^{\perp}(x)\right|^{2} d x\right) \\
& \leqslant \operatorname{ch}^{3}\left(h^{2} \max \left\{1, \mu^{-1}\right\} \mathscr{E}_{\mu}(u ; \Xi)+h \mu^{-1} \mathscr{E}_{\mu}\left(u^{j} ; G_{h}^{j}\right)\right) \\
& \leqslant c h^{4} \max \left\{1, \mu^{-1}\right\} \mathscr{E}_{\mu}(u ; \Omega(h)) .
\end{aligned}
$$

Замораживая подынтегральные выражения в точке $P^{j}$ и учитывая гладкость границы $\partial \Xi$, видим, что матрицы $M^{j}$ из левой части формулы $\left(3.7_{1}\right)$ удовлетворяют соотношениям

$$
\left(\operatorname{mes}_{3} g_{h}^{j}\right)^{-1}\left\|M^{j}-\operatorname{mes}_{3} g_{h}^{j} d\left(P^{j}\right)^{\top} \nu^{j}\left(\nu^{j}\right)^{\top} d\left(P^{j}\right) ; \mathbb{R}^{6} \rightarrow \mathbb{R}^{6}\right\|=: \gamma_{j}(h) \rightarrow 0
$$

при $h \rightarrow+0$. Поскольку $0<c h^{3} \leqslant \operatorname{mes}_{3} g_{h}^{j} \leqslant C h^{3}$, предположение $\left.1^{\circ}\right)$, принятое в начале раздела, обеспечивает оценку $\left\|M^{-1} ; \mathbb{R}^{6} \rightarrow \mathbb{R}^{6}\right\| \leqslant c h^{-3}$ при $h \in\left(0, h_{0}\right]$. Действительно, справедлива формула

$$
\sum b^{\top} d\left(P^{j}\right)^{\top} \nu^{j}\left(\nu^{j}\right)^{\top} d\left(P^{j}\right) b \geqslant c_{0}|b|^{2}, \quad c_{0}>0
$$

(иначе строки $\left(\nu^{j}\right)^{\top} d\left(P^{j}\right) \in \mathbb{R}^{6}, j=1, \ldots, J$, линейно зависимы). Поэтому

$$
b^{\top} M b=\sum_{j=1}^{J} b^{\top} M^{j} b \geqslant \sum_{j=1}^{J} \operatorname{mes}_{3} g_{h}^{j}\left\{\left|\left(\nu^{j}\right)^{\top} d\left(P^{j}\right)\right|^{2}-c \gamma_{j}(h)|b|^{2}\right\} \geqslant c_{1} h^{3}|b|^{2},
$$

если только граница $h_{0}>0$ изменения параметра $h$ выбрана так, что $c_{0}-$ $c\left(\gamma_{1}(h)+\cdots+\gamma_{J}(h)\right) \geqslant c_{0} / 2$ при $h \in\left(0, h_{0}\right]$. Теперь из полученных формул выводим, что

$$
\begin{aligned}
\left\|d b ; H^{1}(\Xi(h))\right\|^{2} & \leqslant c|b|^{2} \leqslant c\left\|M^{-1} ; \mathbb{R}^{6} \rightarrow \mathbb{R}^{6}\right\|^{2}|f|^{2} \\
& \leqslant c h^{-2} \max \left\{1, \mu^{-1}\right\} \mathscr{E}_{\mu}(u ; \Omega(h)) .
\end{aligned}
$$

Благодаря последним неравенствам (3.5) имеем

$$
\left\|u^{0} ; H^{1}(\Xi(h))\right\|^{2} \leqslant c\left(\left\|u^{\perp} ; H^{1}(\Xi(h))\right\|^{2}+|b|^{2}\right) .
$$

Итак, проверено следующее утверждение.

ТеОРема 3.1 [22], [23]. Пусть $J \geqslant 6$ и для сочленения (3.1) выполнено условие $\left.1^{\circ}\right)$. Тогда поле $u \in \stackrel{\circ}{H^{1}}\left(\Omega(h) ; \Gamma_{h}^{1} \cup \cdots \cup \Gamma_{h}^{J}\right)^{3}$ удовлетворяет весовому анизотропному неравенству Корна

$$
\sigma(h, \mu)\left(\left\|\rho^{-1} u^{0} ; L_{2}(\Xi(h))\right\|^{2}+\left\|\nabla_{x} u^{0} ; L_{2}(\Xi(h))\right\|^{2}\right)+\mu \sum_{j=1}^{J} \mid u^{j} ; G_{h}^{j} \mathbf{I}_{j}^{2} \leqslant c \mathscr{E}_{\mu}\left(u ; \Omega_{h}\right),
$$


где $\sigma(h, \mu)=h \min \{1, h \mu\}, \rho(x)=\min \left\{\left|x-P^{j}\right| \mid j=1, \ldots, J\right\}, a \mid u^{j} ; G_{h}^{j} \mathbf{|}_{j}$ норма (2.52), в которой $G_{h}=G_{h}^{j} u \rho_{h}\left(z^{j}\right)=h+\left|z^{j}-l_{j}\right|$.

Если безразмерные параметры $h$ и $\mu$ подчинены соотношению $h \mu \leqslant 1$ (соотношению $h \mu>1$ ), то стержни называются ${ }^{7}$ мягкими (жесткими) в сравнении с телом $\Xi(h)$. Для мягких стержней мажорантой для нормы $\left\|u^{0} ; H^{1}(\Xi(h))\right\| \sim$ $\left\|\rho^{-1} u^{0} ; L_{2}(\Xi(h))\right\|+\left\|\nabla_{x} u^{0} ; L_{2}(\Xi(h))\right\|$ служит величина $c h^{2} \mu^{-1} \mathscr{E}_{\mu}\left(u ; \Omega_{h}\right)$, а для жестких - величина $\mathrm{ch}^{-1} \mathscr{E}_{\mu}\left(u ; \Omega_{h}\right)$.

Пример 3.2. 1) Пусть $b \in \mathbb{R}^{6} \backslash\{0\}$ и $d(x) b$-жесткое смещение, а $d\left(y^{j}, z^{j}\right) b^{j}-$ запись этого смещения в декартовых координатах $\left(y^{j}, z^{j}\right)$, соотнесенных со стержнем $G_{h}^{j}$. Связь столбцов $b$ и $b^{j}$ указана леммой 2.1 , но далее понадобится только очевидное равенство

$$
b_{3}^{j}=\left(\nu^{j}\right)^{\top} d\left(P^{j}\right) b
$$

Положим $u^{0}(x)=d(x) b$ при $x \in \Xi(h)$ и продолжим поле $u^{0}$ на $G_{h}^{j}$ по формулам

$$
\begin{aligned}
& u_{1}^{j}\left(y^{j}, z^{j}\right)=\left(b_{1}^{j}+\alpha z^{j} b_{5}^{j}-\alpha y_{2}^{j} b_{6}^{j}\right) \chi_{j}\left(z^{j}\right), \\
& u_{2}^{j}\left(y^{j}, z^{j}\right)=\left(b_{2}^{j}-\alpha z^{j} b_{4}^{j}+\alpha y_{1}^{j} b_{6}^{j}\right) \chi_{j}\left(z^{j}\right), \\
& u_{3}^{j}\left(y^{j}, z^{j}\right)=b_{3}^{j} \chi_{j}\left(z^{j}\right)-\left(y_{1}^{j} b_{1}^{j}-y_{2}^{j} b_{2}^{j}\right) \frac{\partial}{\partial z^{j}} \chi_{j}\left(z^{j}\right)-\alpha\left(y_{1}^{j} b_{5}^{j}-y_{2}^{j} b_{4}^{j}\right) \frac{\partial}{\partial z^{j}}\left(z^{j} \chi_{j}\left(z^{j}\right)\right) .
\end{aligned}
$$

Здесь $\chi_{j}\left(z^{j}\right)=\chi\left(\left[2 l_{j}\right]^{-1} z^{j}\right)-$ срезающая функция, равная единице при $z^{j} \leqslant l_{j} / 2$ и нулю при $z^{j} \geqslant l_{j}\left(\chi-\right.$ эталонная срезка). Отметим, что $u^{j}\left(y^{j}, l_{j}\right)=0$, так как $\chi\left(l_{j}\right)=\partial_{z^{j}} \chi\left(l_{j}\right)=0$. Конструкция (3.12) аналогична анзацу $\left(2.53_{1}\right)-\left(2.53_{3}\right)$, в котором $w_{q}$ - линейные функции, умноженные на срезку $\chi_{j}$, и поэтому, повторив вычисления, проведенные в примере 2.5 , обнаруживаем, что при некоторых постоянных $c_{j}$ и $C_{j}$ верны соотношения

$$
\begin{gathered}
\left|u^{j} ; G_{h}^{j}\right|_{j}^{2} \geqslant c_{j} h^{2}\left\{\left|b_{3}^{j}\right|^{2}+h^{2}\left(\left|b^{j}\right|^{2}-\left|b_{3}^{j}\right|^{2}\right)\right\}, \\
\mathscr{E}\left(u ; G_{h}^{j}\right) \leqslant C_{j} h^{2}\left\{\left|b_{3}^{j}\right|^{2}+h^{2}\left(\left|b^{j}\right|^{2}-\left|b_{3}^{j}\right|^{2}\right)\right\} .
\end{gathered}
$$

Поскольку $u^{0}=\left.u\right|_{\Xi(h)}$ - жесткое смещение, имеем

$$
\mathscr{E}\left(u^{0} ; \Xi(h)\right)=0, \quad\left\|u^{0} ; L_{2}(\Xi(h))\right\|^{2} \geqslant c_{0}|b|^{2}, \quad\left\|\nabla_{x} u^{0} ; L_{2}(\Xi(h))\right\|^{2} \geqslant C_{0}\left|b^{\prime \prime}\right|^{2} .
$$

В согласии со связью (3.11) условие $1^{\circ}$ ) означает, что хотя бы одна из компонент $b_{3}^{1}, \ldots, b_{3}^{J}$ отлична от нуля. Следовательно, правая часть (3.10) не превосходит $c \mu h^{2}$, а левая часть (3.10) больше, чем $c h(\min \{1, h \mu\}+h \mu)$. Итак, для мягких стержней $(h \mu \leqslant 1)$ асимптотическая точность неравенства (3.10) подтверждена.

2) Если стержни жесткие, то возьмем такое поле:

$$
\begin{aligned}
u^{0}(x) & =X_{h}(x) d(x) b, & & x \in \Xi(h), \\
u^{j}(x) & =0, & & x \in G_{h}^{j}, \quad j=1, \ldots, J .
\end{aligned}
$$

Здесь $X_{h}=1-\chi_{h}^{1}-\cdots-\chi_{h}^{J}$ и $\chi_{h}^{j}-$ гладкие срезающие функции, причем $\chi_{h}^{j}(x)=\chi\left(\left[2 \mathbf{r}_{j}\right]^{-1}\left|x-P^{j}\right|\right)$, а радиус $\mathbf{r}_{j}$ выбран так, чтобы $\chi_{h}^{j}(x)=1$ при $x \in g_{h}^{j}$.

\footnotetext{
${ }^{7}$ Более правильно говорить о жесткости стержней на растяжение.
} 
Для поля $\left(3.15_{1}\right)$ выполнены соотношения (2.22). Повторив выкладку из примеpa 2.2 , видим, что $\mathscr{E}_{\mu}(u ; \Omega(h))=\mathscr{E}\left(u^{0} ; \Xi(h)\right) \leqslant c h|b|^{2}$. Теперь левая часть $(3.10)$ оценивается снизу величиной $c h \min \{1, h \mu\}$, а правая часть (3.10) мажорируется произведением $C h$, т. е. при $h \mu>1$ асимптотическая точность неравенства Корна (3.10) сомнений не вызывает.

3) Если условие $1^{\circ}$ ) нарушено, то найдется нетривиальный столбец $b \in \mathbb{B}^{6}$, для которого $b_{3}^{j}=0$ при $j=1, \ldots, J$. Теперь в соответствии с формулами (3.13) и (3.14) справедливость неравенства (3.10) влечет за собой соотношение $h \min \{1, h \mu\}+\mu h^{4} \leqslant C \mu h^{4}$, невозможное при $\mu h \leqslant 1$. Итак, геометрическое условие $1^{\circ}$ ) является необходимым.

3.3. Произвольное расположение стержней. Рассмотрим сочленение (3.1) без каких-либо ограничений на расположение и количество стержней. Пусть $\mathscr{C}_{h}^{1} \subset g_{h}^{1}=\Xi \cap G_{h}^{1}-$ круговой цилиндр с высотой $2 R h$, радиусом основания $R h$ и осью вращения, параллельной оси $z^{1}=x_{3}$. Пусть еще $\mathscr{G}_{h}^{1-}$ цилиндр того же радиуса, но длиной $l_{j}^{0}$, не зависящей от $h$, причем $\mathscr{G}_{h}^{1} \subset G_{h}^{1}$ и $\mathscr{C}_{h}^{1} \subset \mathscr{G}_{h}^{1}$. В силу теорем $2.10,2.9$ и следствия 2.2 верна оценка

$$
\begin{aligned}
h^{-1}\left\|u_{i}-\bar{u}_{i} ; L_{2}\left(\mathscr{C}_{h}^{1}\right)\right\|^{2} & \leqslant c\left(\mathscr{E}\left(u ; \mathscr{G}_{h}^{1}\right)+\left|u ; \mathscr{G}_{h}^{1}\right|^{2}\right) \\
& \leqslant c\left(\mathscr{E}\left(u ; G_{h}^{1}\right)+\left|u ; G_{h}^{1}\right|_{1}^{2}\right) \leqslant c \mathscr{E}\left(u ; G_{h}^{1}\right) .
\end{aligned}
$$

Воспользуемся разложением $\left(2.58_{1}\right)$ поля $u$ на $\Xi$, но в соответствии с леммой 2.1 перепишем жесткое смещение $d(x) b$ в виде $d(\mathbf{y}, \mathbf{z}) \mathbf{b}$; при этом центр системы декартовых координат $(\mathbf{y}, \mathbf{z})$ совместим с центром тяжести цилиндра $\mathscr{C}_{h}^{1}$, а ось $\mathbf{z}$ направим вдоль его оси вращения.

Оценим столбец $\mathbf{b} \in \mathbb{R}^{6}$. С этой целью умножим равенство $\left(2.58_{1}\right)$ на $(6 \times 3)$ матрицу $d(\mathbf{y}, \mathbf{z})^{\top}$ и проинтегрируем по цилиндру $\mathscr{C}_{h}$. В результате получаем представление

$$
\mathbf{b}=\mathbf{d}\left(\mathscr{C}_{h}^{1}\right)^{-1} \int_{\mathscr{C}_{h}^{1}} d(\mathbf{y}, \mathbf{z})^{\top}\left(u(x)-u^{\perp}(x)\right) d \mathbf{y} d \mathbf{z}
$$

где диагональная $(6 \times 6)$-матрица $\mathbf{d}\left(\mathscr{C}_{h}^{1}\right)$ для цилиндра высотой $H=R h$ имеет вид

$$
\mathbf{d}\left(\mathscr{C}_{h}^{1}\right)=2 \pi R^{2} h^{2} H \operatorname{diag}\left\{1,1,1, \frac{1}{6} H^{2}+\frac{1}{8} R^{2} h^{2}, \frac{1}{6} H^{2}+\frac{1}{8} R^{2} h^{2}, \frac{1}{4} R^{2} h^{2}\right\} .
$$

Сначала, как и в выкладке (3.8), учитывая неравенства (2.55) и (2.32) при $\delta=0$, выводим оценки первых трех компонент:

$$
\begin{aligned}
\left|\mathbf{b}_{i}\right|^{2} & \leqslant c h^{-6} \operatorname{mes}_{3} \mathscr{C}_{h}^{1} \int_{\mathscr{C}_{h}^{1}}\left(\left|u_{i}^{j}\right|^{2}+\left|u^{\perp}\right|^{2}\right) d x \\
& \leqslant c h^{-3}\left(h^{-1}\left|u^{j} ; G_{h}^{1}\right|_{1}^{2}+h^{2}\left\|\rho^{-1} u^{\perp} ; L_{2}\left(\mathscr{C}_{h}^{1}\right)\right\|^{2}\right) \\
& \leqslant c h^{-1} \max \left\{h^{-3} \mu^{-1}, 1\right\} \mathscr{E}_{\mu}(u ; \Omega(h)), \quad i=1,2, \\
\left|\mathbf{b}_{3}\right|^{2} & \leqslant c h^{-1} \max \left\{h^{-1} \mu^{-1}, 1\right\} \mathscr{E}_{\mu}(u ; \Omega(h)) .
\end{aligned}
$$


При обработке очередных двух компонент $\mathbf{b}_{4}$ и $\mathbf{b}_{5}$ используем функцию $\mathbf{z} \mapsto$ $\psi(\mathbf{z})=\frac{1}{6} \mathbf{z}\left(\mathbf{z}^{2}-2 R^{2} h^{2}\right)$. Поскольку $\partial_{\mathbf{z}}^{2} \psi(\mathbf{z})=\mathbf{z}$ и $\partial_{\mathbf{z}} \psi( \pm R h)=0$, находим

$$
\begin{aligned}
\left|\mathbf{b}_{6-i}\right|^{2} & \leqslant c h^{-10}\left(\left|\int_{\mathscr{C}_{h}^{1}}\left(u_{i}^{j} \partial_{\mathbf{z}}^{2} \psi-\mathbf{y}_{i} u_{3}^{j}\right) d \mathbf{y} d \mathbf{z}\right|^{2}+h^{2}\left|\int_{\mathscr{C}_{h}^{1}}\right| u^{\perp}|d \mathbf{y} d \mathbf{z}|^{2}\right) \\
& \leqslant h^{-7}\left(h^{3} \mu^{-1}+h^{4} \max \left\{1, \mu^{-1}\right\}\right) \mathscr{E}_{\mu}(u ; \Omega(h)) \\
& \leqslant c h^{-3} \max \left\{h^{-1} \mu^{-1}, 1\right\} \mathscr{E}_{\mu}(u ; \Omega(h)), \quad i=1,2 .
\end{aligned}
$$

Наконец, неравенство (3.16) обеспечивает оценку компоненты $\mathbf{b}_{6}$, а именно,

$$
\begin{aligned}
\left|\mathbf{b}_{6}\right|^{2} & \leqslant c h^{-10}\left(\left|\int_{\mathscr{C}_{h}^{1}}\left(\mathbf{y}_{2}\left(u_{1}^{j}-\bar{u}_{1}^{j}\right)-\mathbf{y}_{1}\left(u_{2}^{j}-\bar{u}_{2}^{j}\right)\right) d \mathbf{y} d \mathbf{z}\right|^{2}+h^{2} \operatorname{mes}_{3} \mathscr{C}_{h}^{1} \int_{\mathscr{C}_{h}}\left|u^{\perp}\right|^{2} d x\right) \\
& \leqslant c h^{-7}\left(h^{2} \sum_{i=1}^{2}\left\|u_{i}^{j}-\bar{u}_{i}^{j} ; L_{2}\left(\mathscr{C}_{h}^{1}\right)\right\|^{2}+h^{4}\left\|\rho^{-2} u^{\perp} ; L_{2}\left(\mathscr{C}_{h}^{1}\right)\right\|^{2}\right) \\
& \leqslant c h^{-7}\left(h^{3} \mu^{-1}+h^{4} \max \left\{1, \mu^{-1}\right\}\right) \mathscr{E}_{\mu}(u ; \Omega(h)) \\
& \leqslant c h^{-3} \max \left\{h^{-1} \mu^{-1}, 1\right\} \mathscr{E}_{\mu}(u ; \Omega(h)) .
\end{aligned}
$$

Пояснение: компоненту $u_{i}^{j}$ удалось заменить разностью $u_{i}^{j}-\bar{u}_{i}^{j}$ потому, что интеграл от произведения переменной $\mathbf{y}_{3-i}$ и ее среднего $\bar{u}_{i}^{j}$ по цилиндру $\mathscr{C}_{h}^{1}$ равен нулю. При этом неравенство Пуанкаре

$$
\left\|u_{i}^{j}-\bar{u}_{i}^{j} ; L_{2}\left(\mathscr{C}_{h}^{1}\right)\right\| \leqslant \operatorname{ch}\left\|\nabla_{x}\left(u_{i}^{j}-\bar{u}_{i}^{j}\right) ; L_{2}\left(\mathscr{C}_{h}^{1}\right)\right\|=\operatorname{ch}\left\|\nabla_{x} u_{i}^{j} ; L_{2}\left(\mathscr{C}_{h}^{1}\right)\right\|
$$

привносит в правую часть (3.19) дополнительный множитель $h^{2}$.

Принимая во внимание оценку (3.9) и соотношение $|b| \leqslant c|\mathbf{b}|$, обеспеченное леммой 2.1, приходим к очередному утверждению.

ТеОрема 3.2 [22]. Для поля $u \in \stackrel{\circ}{H}^{1}\left(\Omega(h) ; \Gamma_{h}^{1} \cup \cdots \cup \Gamma_{h}^{J}\right)^{3}$ справедливо весовое анизотропное неравенство Корна (3.10), где $\sigma(h, \mu)=h^{3} \min \{1, h \mu\}$, а остальные обозначения такие же, как и в теореме 3.1 .

Пусть имеются три точки, например, $P^{1}, P^{2}$ и $P^{3}$, лежащие в вершинах невырожденного треугольника. Поместим начало координат $x$ в точку $P^{1}$, а оси $x_{1}$ и $x_{3}$ направим соответственно в точку $P^{2}$ и перпендикулярно плоскости, содержащей $P^{1}, P^{2}$ и $P^{3}$. Обозначим $b^{j}$ столбцы, получающиеся после перезаписи жесткого смещения $d(x) b$ в координатах $\left(y^{j}, z^{j}\right)$. Повторим выкладки (3.18), (3.19) для столбцов $\mathbf{b}^{j}$ и цилиндров $\mathscr{C}_{h}^{j} \subset g_{h}^{j}$. Поскольку расстояние от центра тяжести цилиндра $\mathscr{C}_{h}^{j}$ до точки $P^{j}$ составляет $O(h)$, благодаря лемме 2.1, указывающей связь столбцов $b^{j}$ и $\mathbf{b}^{j}$, и неравенствам (3.18), (3.19) имеем

$$
\left|b^{j \prime}\right|^{2} \leqslant c\left(\left|\mathbf{b}^{j \prime}\right|^{2}+h^{2}\left|\mathbf{b}^{j \prime \prime}\right|^{2}\right) \leqslant c h^{-1} \max \left\{h^{-3} \mu^{-1}, 1\right\} \mathscr{E}_{\mu}(u ; \Omega(h)) .
$$

Согласно той же лемме, $b^{q \prime}-b^{1 \prime}=d^{\prime \prime}\left(x^{1 q}\right) b^{1 \prime \prime}$ и

$$
d^{\prime \prime}\left(x^{12}\right)=\left(\begin{array}{ccc}
0 & 0 & 0 \\
0 & 0 & \alpha x_{1}^{12} \\
0 & -\alpha x_{1}^{12} & 0
\end{array}\right), \quad d^{\prime \prime}\left(x^{13}\right)=\left(\begin{array}{ccc}
0 & 0 & -\alpha x_{2}^{13} \\
0 & 0 & \alpha x_{1}^{13} \\
\alpha x_{2}^{13} & -\alpha x_{1}^{13} & 0
\end{array}\right)
$$


Здесь $x_{i}^{1 q}-$ координаты точки $P^{q}$ в системе $x^{1}$. Отсюда и из оценки $(3.20)$ выводим, что

$$
\left|b_{4}^{1}\right|^{2}+\left|b_{5}^{1}\right|^{2} \leqslant c\left(\left|b^{2 \prime}\right|^{2}+\left|b^{1 \prime}\right|^{2}\right), \quad\left|b_{6}^{1}\right|^{2} \leqslant c\left(\left|b^{3 \prime}\right|^{2}+\left|b^{1 \prime}\right|^{2}+\left|b_{4}^{1}\right|^{2}+\left|b_{5}^{1}\right|^{2}\right) .
$$

Совмещая неравенства $(3.20)$, (3.21) и (3.9), формулируем еще одно утверждение.

ТЕОРема 3.3 [22]. Пусть $J \geqslant 3$ и выполнено условие треугольника $2^{\circ}$ ) из $\S 2$. Тогда для поля $u \in \stackrel{\circ}{H}^{1}\left(\Omega(h) ; \Gamma_{h}^{1} \cup \cdots \cup \Gamma_{h}^{J}\right)^{3}$ справедливо весовое анизотропное неравенство Корна $(3.10)$, где $\sigma(h, \mu)=h \min \left\{1, h^{3} \mu\right\}$, а остальные обозначения такие же, как и в теореме 3.1 .

Множители при выражении, отделенном в (3.10) скобками и эквивалентном квадрату нормы $\left\|u^{0} ; H^{1}(\Xi(h))\right\|$, разнятся в теоремах $3.1,3.2$, но связаны соотношениями

$$
h \min \{1, h \mu\} \geqslant h \min \left\{1, h^{3} \mu\right\} \geqslant h^{3} \min \{1, h \mu\} .
$$

Если стержни очень жесткие ${ }^{8}$ и $h^{3} \mu>1$, то строка (3.22) читается так: $h=$ $h \geqslant h^{3}$. Следовательно, для обеспечения наибольшей нормы в левой части неравенства Корна (3.10) требование $1^{\circ}$ ) заполнения пространства $\mathbb{R}^{6}$ не нужно - достаточно условия треугольника $\left.2^{\circ}\right)$.

Если стержни просто жесткие $\left(h \mu>1\right.$, но $\left.h^{3} \mu \ll 1\right)$, то строка (3.22) принимает вид $h \geqslant h^{4} \mu \geqslant h^{3}$, а значит, каждое из двух условий влияет на качество оценки (3.10).

Наконец, при мягких стержнях $(h \mu \leqslant 1)$ вместо формулы (3.22) получаем такую: $h^{2} \mu \geqslant h^{4} \mu=h^{4} \mu$. Следовательно, конструкция (3.1) безразлична к условию треугольника $2^{\circ}$ ), а наилучшая норма обеспечивается условием $1^{\circ}$ ). Эти факты подтверждают сказанное в п. 2.2.

Конкретные поля смещений, упомянутые в примере $3.2,3$ ) и 2), указывают на асимптотическую точность неравенств Корна из теорем 3.2 и 3.3. Необходимость условия треугольника, затребованного в теореме 3.3 , проверяется рассмотрением вектор-функции $u^{\theta}$ из замечания 2.3, уже встречавшейся в п. 2.2.

Для однородных $(\mu=1)$ сочленений асимптотический анализ проводился в публикациях [20], [21], [43] (изотропный упругий материал) и в статьях [44]-[46] (произвольная анизотропия упругих материалов). В [24] изучались конструкции (3.1), изготовленные из контрастных материалов, а именно, $\mu=h^{s}$ при $s \in \mathbb{R} \backslash\{0\}$. Изменение асимптотических анзацев в случае выполнения условия $1^{\circ}$ ) из п. 3.2 впервые обнаружено в работе [45].

ЗАмечАниЕ 3.1. Утверждения в теореме 2.3 проверяются по той же схеме, что и теоремы 3.1 и 3.3. Впрочем, их можно получить как следствия этих теорем, считая стержни $G_{h}^{1}, \ldots, G_{h}^{J}$ достаточно жесткими и обеспечивая условия $\left(2.18_{1}\right)$ или $\left(2.18_{2}\right)$ при помощи неравенства $(2.55)$ и умножения поля смещений на подходящую срезающую функцию.

\footnotetext{
${ }^{8}$ Более правильно говорить о жесткости стержней на изгиб, которая должна быть много меньше жесткости самого тела.
} 
3.4. Пронизывающие стержни. Примем все обозначения из п. 3.1 , за исключением определения стержней $G_{h}^{j}$. Именно, считаем, что стержни имеют вид

$$
G_{h}^{j}=\left\{x: z^{j} \in\left(-l_{j}^{0}, l_{j}\right), h^{-1} y^{j} \in \omega^{j}\right\}
$$

и глубоко проникают вовнутрь тела, т.е. $l_{j}^{0}>0$ - фиксированные длины, не зависящие от параметра $h \in\left(0, h_{0}\right]$. Без ограничения общности предполагаем, что начало координат $y^{j}$ содержится в области $\omega^{j}$. Обозначим $\mathscr{C}_{h}^{j}=\{x$ : $\left.\left|y^{j}\right|<R h,\left|z^{j}-z_{0}^{j}\right|<H\right\}$ круговой цилиндр, содержащийся в стержне $G_{h}^{j}$, a $\Upsilon^{j}-$ оси $\left\{x: y^{j}=0, z^{j} \in\left(-l_{j}^{0}, 0\right)\right\}$ гнезд $g_{h}^{j}=G_{h}^{j} \cap \Xi$.

ТЕОрема 3.4 [22]. Для поля $u \in \stackrel{\circ}{H^{1}}\left(\Omega(h) ; \Gamma_{h}^{1} \cup \cdots \cup \Gamma_{h}^{J}\right)^{3}$ справедливо весовое анизотропное неравенство Корна

$$
\begin{gathered}
\sigma(h, \mu)\left(\left\|\rho^{-1}(1+|\log \rho|)^{-1} u^{0} ; L_{2}(\Xi(h))\right\|^{2}+\left\|\nabla_{x} u^{0} ; L_{2}(\Xi(h))\right\|^{2}\right) \\
+\mu \sum_{j=1}^{J}\left|u^{j} ; G_{h}^{j}\right|_{j}^{2} \leqslant c \min \{1, \mu\} \mathscr{E}_{\mu}\left(u ; \Omega_{h}\right),
\end{gathered}
$$

где $\rho(x)=\min \left\{\operatorname{dist}\left(x, \Upsilon^{j}\right) \mid j=1, \ldots, J\right\}$, а множитель $\sigma(h, \mu)$ определен следующим образом:

1) $\sigma(h, \mu)=\min \left\{(1+|\log h|)^{-2}, h^{2} \mu\right\}$ при условии $\left.1^{\circ}\right)$;

2) если $J \geqslant 2$ и имеются два стержня, оси которых не попадают на одну прямую, $\operatorname{mo} \sigma(h, \mu)=\min \left\{(1+|\log h|)^{-2}, h^{4} \mu\right\}$;

3) если $J=1$ или все точки $P^{1}, \ldots, P^{J}$ лежат на одной прямой, а векторь $\nu^{1}, \ldots, \nu^{J}$ направлены вдоль нее, $\operatorname{mo} \sigma(h, \mu)=h^{2} \min \left\{1, h^{2} \mu\right\}$.

Заметим, что в теореме $3.4,2$ ) неявно задействовано условие треугольника: на несовпадающие оси двух стержней всегда можно поместить вершины невырожденного треугольника.

Множители $\sigma(h, \mu)$ из теоремы 3.4 связаны соотношениями

$$
\min \left\{(1+|\log h|)^{-2}, h^{2} \mu\right\} \geqslant \min \left\{(1+|\log h|)^{-2}, h^{4} \mu\right\} \geqslant h^{2} \min \left\{1, h^{2} \mu\right\} .
$$

Каждая из величин в списке (3.25) не меньше соответствующей величины в списке (3.22). Неравенства (3.25) устанавливают иерархию: мягкие, просто жесткие и очень жесткие стержни. При этом ограничения на $h$ и $\mu$ отличаются от указанных в предыдущем пункте.

Пример 3.3. 1) Шашлык. Пусть $\Xi-$ куб $\left\{x:\left|x_{p}\right|<1, p=1,2,3\right\}$, а $G_{h}^{1}-$ цилиндр $\left\{x=\left(x^{\prime}, x_{3}\right):\left|x^{\prime}\right|<h,\left|x_{3}\right|<2\right\}$ (см. рис. 5). Если "шампур" $G_{h}^{1}$ жесткий и $h^{2} \mu \geqslant 1$, то точность неравенства (3.24) при $\sigma(h, \mu)=h^{2}$ подтверждается полем $u^{\theta}$ из замечания 2.3. Это же поле демонстрирует необходимость условия теоремы $3.4,2)$. Если стержень мягкий и $h^{2} \mu \leqslant 1$, то нужное поле имеет вид $u(x)=\theta(y) \chi\left(x_{3}\right)$, так как $\mathscr{E}(u ; \Xi)=0$ и $\mathscr{E}\left(u ; G_{h}^{1} \backslash \Xi\right)=O\left(h^{4}\right)$.

2) Столярный рейсмус. Пусть $\Xi$ - тот же куб и $G_{h}^{j}=\left\{x:\left|x_{3}\right|<2, x_{1}^{2}+\right.$ $\left.\left(x_{2}-\frac{1}{2}(-1)^{j}\right)^{2}<h^{2}\right\}$ (см. рис. 6). Для очень жестких стержней (т. е. $h^{4} \mu>$ $\left.(1+|\log h|)^{-2}\right)$ любое жесткое смещение из линеала $\mathfrak{R}$, умноженное на срезки $\chi_{h}^{j}(x)=\chi_{-}\left((\log h)^{-1} \log \left|y^{j}\right|\right)$, где $y_{1}^{j}=x_{1}$ и $y_{2}^{j}=x_{2}-\frac{1}{2}(-1)^{j}$, показывает, 


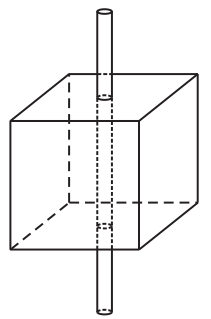

Рис. 5. Шашлык

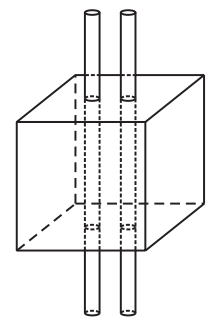

Рис. 6. Столярный рейсмус

что неравенство (3.24) при $\sigma(h, \mu)=(1+|\log h|)^{-1}$ неулучшаемо (ср. замечание $2.3,1))$. Эта же конструкция годится при любом расположении стержней, в частности, для случая, указанного теоремой $3.4,1)$.

При $h^{4} \mu \leqslant(1+|\log h|)^{-2}$ для вектор-функций $u^{i}(x)=e_{i} \chi\left(x_{3}\right)-x_{i} e_{3} \partial_{x_{3}} \chi\left(x_{3}\right)$, $i=1,2$, как и в предыдущем примере, имеем $\mathscr{E}(u ; \Xi)=0$ и $\mathscr{E}\left(u ; G_{h}^{j} \backslash \Xi\right)=O\left(h^{4}\right)$.

3) Клубок с вязальными спицами. Пусть стержни мягкие, т. е. $h^{2} \mu<(1+$ $|\log h|)^{-2}$, но условие $1^{\circ}$ ) нарушено (например, $\left.J \leqslant 5\right)$. Тогда найдется жесткое смещение $u^{0}(x)=d(x) b$, для которого все величины (3.11) обращаются в нуль, а продолжение поля $u^{0}$ на "спицы" $G_{h}^{j}$ по формулам (3.12) показывает, что $\mathscr{E}_{\mu}(u ; \Omega(h))=O\left(h^{4} \mu\right)$, т. е. условие заполнения пространства $\mathbb{R}^{6}$ необходимо для выполнения неравенства (3.24) при $\sigma(h, \mu)=h^{2} \mu$.

Асимптотический анализ задачи теории упругости для тела, пронзенного двумя очень жесткими стержнями (пример $3.3,2)$ ), проводился в работах [47] и [48]. Задачи теории осреднения для армированных тел изучались в статьях [49], [50], в книге [51; гл. 7] и др.

3.5. Незащемленные стержни. Пусть торцы $\omega_{h}^{j}\left(l_{j}\right)$ стержней $G_{h}^{j}$ свободны, а закрепление конструкции (3.1) производится, например, по части Г поверхности $\partial \Xi$, удаленной от гнезд $g_{h}^{j}$, т. е. $\operatorname{mes}_{2} \Gamma>0$ и $P^{j} \neq \bar{\Gamma}$ при $j=1, \ldots, J$.

Неравенство Корна на теле $\Xi$ необходимо распространить на стержни. Этот вопрос изучался в работе [11], где рассматривались однородные $(\mu=1)$ сочленения и использовались изотропные соболевские нормы. Замещая последние анизотропными весовыми нормами и повторяя с упрощениями проведенные выше выкладки, усиливаем результат.

Теорема 3.5. Для поля $u \in \stackrel{\circ}{H}^{1}(\Omega(h) ; \Gamma)^{3}$ справедливо неравенство Корна

$$
\min \{1, \mu\}\left\|u ; H^{1}(\Xi)\right\|^{2}+\beta(h, \mu) \sum_{j=1}^{J}\left|u^{j} ; G_{h}^{j}\right|^{2} \leqslant c \mathscr{E}_{\mu}(u ; \Omega(h)),
$$

где $\left|u ; G_{h}\right|$ - норма (2.52) при $\rho_{h}(z)=1$, а множитель $\beta(h, \mu)$ определяется следуюшим образом:

1) для стержней $G_{h}^{j}$, вставленных в неглубокие гнезда, $\beta(h, \mu)=\min \{1, \mu\}$;

2) для стержней (3.23), пронизывающих тело, $\beta(h, \mu)=h^{-2} \min \{(1+$ $\left.|\log h|)^{-2}, h^{2} \mu\right\}$. 
Асимптотическая точность неравенства (3.26) проверяется подстановкой в него поля смещений $\mathscr{X}_{h}^{j}(x) d(x) b$, где $d b$ - жесткое смещение, а $\mathscr{X}_{h}^{j}$ - срезающая функция, равная единице на множестве $G_{h}^{j}$ и нулю вне его $h$-окрестности. Если глубина гнезд составляет $O(h)$, то в соответствии с предложением 2.2 замена нормы $\left\|u ; H^{1}(\Xi)\right\|$ нормой $\left\|u ; H^{1}(\Xi(h))\right\|$ (см. определение (3.2)) позволяет убрать множитель $\min \{1, \mu\}$. Исследования постоянных в неравенстве Корна для тел с глубокими скважинами или длинными бороздами вдоль поверхности не проводились.

\section{§ 4. Неравенства Корна для сочленения массивного тела с пластинами}

4.1. Геометрия сочленений. Пусть $\Xi \in \mathbb{R}^{3}$ - область с гладкой границей $\partial \Xi$ и компактным замыканием $\Xi=\Xi \cup \partial \Xi$. Зафиксируем плоскости $\Pi^{1}, \ldots, \Pi^{J}$ и свяжем с $\Pi^{j}$ систему декартовых координат $\left(y_{1}^{j}, y_{2}^{j}, z^{j}\right)$, направив ось $z^{j}$ перпендикулярно этой плоскости. Пусть еще $\omega^{j}-$ область на плоскости $\Pi^{j}$ с границей $\partial \omega^{j}$, причем для трехмерного множества $\left\{x: y^{j}=\right.$ $\left.\left(y_{1}^{j}, y_{2}^{j}\right) \in \omega^{j}, z^{j}=0\right\}$ используем тот же символ $\omega^{j}$. Иными словами, не будем различать в обозначениях двумерные объекты и их погружения в пространство $\mathbb{R}^{3}$ на плоскость $\Pi^{j}$. В частности, $\Upsilon^{j}=\partial \Xi \cap \omega^{j}-$ дуга (или объединение дуг) на плоскости и в пространстве. Считаем, что перечисленные множества выбраны так, что $\overline{\omega^{j}} \cap \overline{\omega^{k}}=\varnothing$ при $j \neq k$, а $\omega^{j} \cap \Xi \subset \Pi^{j}$ и $\Upsilon^{j}=\omega^{j} \cap \partial \Xi-$ непустые объединения областей и дуг соответственно. Не исключается случай $\Pi^{j}=\Pi^{k}$ при $j \neq k$; при этом удобно называть "параллельными" и совпадающие плоскости.

Тонкие пластины переменной толщины зададим аналогичной (2.33) формулой

$$
\Omega_{h}^{j}=\left\{x: y^{j}=\left(y_{1}^{j}, y_{2}^{j}\right) \in \omega^{j},-h H_{-}^{j}\left(y^{j}\right)<z^{j}<h H_{+}^{j}\left(y^{j}\right)\right\},
$$

в которой $h \in\left(0, h_{0}\right]$ - малый параметр, а $H_{ \pm}^{j}$ - функции на множестве $\overline{\omega^{j}}$, $H^{j}=H_{+}^{j}+H_{-}^{j}>0$. При малом $h_{0}$ пластины $\Omega_{h}^{j}$ и $\Omega_{h}^{k}$ не пересекаются, если $j \neq k$.

Сочленение $G(h)$ тела $\Xi(h)$ с пронизывающими пластинами имеет вид

$$
\begin{aligned}
& G(h)=\Xi \cup \Omega_{h}^{1} \cup \cdots \cup \Omega_{h}^{J} ; \\
& \Xi(h)=\Xi \backslash\left(\overline{\Omega_{h}^{1}} \cup \cdots \cup \overline{\Omega_{h}^{J}}\right) .
\end{aligned}
$$

Материалы, из которых изготовлены тело с прорезями $\left(4.2_{2}\right)$ и пластины (4.1), считаем контрастными, т. е. рассматриваем подобный (2.56) и (3.2) эрзац упругой энергии

$$
\mathscr{E}_{\mu}(u ; G(h))=\left\|D\left(\nabla_{x}\right) u^{0} ; L_{2}(\Xi(h))\right\|^{2}+\mu \sum_{j=1}^{J}\left\|D\left(\nabla_{x}\right) u^{j} ; L_{2}\left(\Omega_{h}^{j}\right)\right\|^{2} .
$$

Помимо сочленения $\left(4.2_{1}\right)$ будем изучать сочленение $\widetilde{G}(h)$, образованное телом $\widetilde{\Xi}(h)$ и пластинами $\widetilde{\Omega}_{h}^{1}, \ldots, \widetilde{\Omega}_{h}^{J}$, которые вставлены в $n a 3 ы$, т. е. прорези 
глубиной $O(h)$,

$$
\begin{aligned}
& \widetilde{G}(h)=\Xi \cup \Omega_{h}^{1} \cup \cdots \cup \Omega_{h}^{J}, \\
& \widetilde{\Xi}(h)=\Xi \backslash\left(\overline{\widetilde{\Omega}_{h}^{1} \cup \cdots \cup \widetilde{\Omega}_{h}^{J}}\right) .
\end{aligned}
$$

При этом множества $\widetilde{\Omega}_{h}^{j}=\left\{x \in \Omega_{h}^{j}: y^{j} \in \widetilde{\omega}^{j}(h)\right\}$ получены усечением пластин (4.1), а именно, подобласть $\widetilde{\omega}^{j}(h)$ отрезана от области $\omega^{j}$ гладкой дугой $v^{j}(h)=\left\{y^{j}: s^{j} \in \Upsilon^{j}, n^{j}=h H_{0}^{j}\left(s^{j}\right)\right\}$, пересекающей контур $\partial \omega^{j}$ под ненулевыми углами. Здесь $\left(s^{j}, n^{j}\right)$ - криволинейные координаты, $s^{j}-$ длина дуги на $\Upsilon^{j}, n^{j}$ - расстояние до $\Upsilon^{j}$, взятое на множестве $\omega^{j} \backslash \bar{\Xi}$ со знаком минус, а $H_{0}^{j}$ - гладкая положительная функция. Приписав "тильду" к символам $G, \Xi$ и $\Omega$ в определении (4.3), образуем эрзац упругой энергии для сочленения $\left(4.4_{1}\right)$. Подчеркнем, что по определению $\widetilde{\Omega}_{h}^{j}$ - пластина с возмущенной кромкой, удовлетворяющая условию $3^{\circ}$ ) и $4^{\circ}$ ) из п. 2.4, и поэтому для нее справедлива теорема 2.7 .

Множества $\left(4.2_{1}\right)$ и $\left(4.4_{1}\right)$ совпадают, однако сочленения $G(h)$ и $\widetilde{G}(h)$, изготовленные из контрастных материалов, обладают различными свойствами. Далее глубокие прорези $g_{h}^{j}=\Xi \cap \Omega_{h}^{j}$ и мелкие пазы $g_{h}^{j}=\Xi \cap \widetilde{\Omega}_{h}^{j}$ называем гнездами. Сочленение закреплено по частям

$$
\Gamma_{h}^{j}=\left\{x \in \partial \Omega_{h}^{j}: y^{j} \in \gamma^{j},-h H_{-}^{j}\left(y^{j}\right)<z^{j}<h H_{+}^{j}\left(y^{j}\right)\right\}
$$

выступающих из тела $\Xi$ боковых поверхностей пластин; здесь $\gamma^{j}-$ непустая дуга на контуре $\partial \omega^{j}$, причем $\overline{\gamma^{j}} \subset \partial \omega^{j} \backslash \bar{\Xi}$. Иными словами, предполагаются выполненными условия $\left(2.18_{1}\right)$ на объединении $\Gamma^{\circ}(h)$ множеств $(4.5), j=1, \ldots, J$.

Большинство ограничений на строение сочленений и гладкость границ объектов введены здесь для упрощения изложения, но возможны разнообразные обобщения на основе приемов, описанных в 2 (ср. с комментариями в конце п. 3.1).

4.2. Пластины, вставленные в неглубокие пазы. Вывод неравенств Корна проводится в целом так же, как и в 3 , но имеет свои особенности. Помимо замены малых по всем трем измерениям цилиндров удлиненными цилиндрами $\mathscr{C}_{h}^{m} \subset g_{h}^{j}$ с радиусом основания $R h$ и высотой $2 H=O\left(h^{0}\right)$, возникает проблема с оценкой интеграла $I^{m}\left(u^{j}\right)=\int_{\mathscr{C}_{h}^{m}} \mathbf{y}_{2}^{m} u_{3}^{m j}\left(y^{j}, z^{j}\right) d x$, так как элемент $\mathbf{d}_{44}\left(\mathscr{C}_{h}^{m}\right)$ матрицы Грама $(3.17)$ - бесконечно малая $O\left(h^{4}\right)$ более высокого порядка, чем остальные. Поскольку нужное неравенство составляет один из восьми ключевых приемов, отмеченных во введении, приводим его формулировку.

Лемма 4.1 [52]. Справедливо неравенство

$$
\left|I^{m}\left(u^{j}\right)\right|^{2} \leqslant c h^{4}\left(\sum_{i=1}^{2}\left\|u_{i}^{j} ; L_{2}\left(\mathscr{C}_{h}^{m}\right)\right\|^{2}+h^{2} \mathscr{E}_{1}\left(u^{j} ; \mathscr{C}_{h}^{m}\right)\right),
$$

где величина с не зависит от параметра $h \in(0,1]$ и поля $u \in H^{1}\left(\Omega^{j}(h)\right)^{3}$.

Далее будем различать три геометрические ситуации, две из которых описываются условиями, аналогичными требованиям $1^{\circ}$ ) и $2^{\circ}$ ) из п. 2.2 , а именно, 
$\left.1^{\circ}\right) \mathfrak{L}\left\{d(x)^{\top} e_{i}^{j} \mid x \in \Upsilon^{j}, i=1,2, j=1, \ldots, J\right\}=\mathbb{R}^{6}$,

$\left.2^{\circ}\right) \mathfrak{L}\left\{x \in \Upsilon^{j} \mid j=1, \ldots, J\right\}=\mathbb{R}^{3}$.

Теорема 4.1 [52]. Для поля $u \in \stackrel{\circ}{H^{1}}(G(h) ; \Gamma(h))^{3}$ справедливо неравенство Корна

$$
\begin{gathered}
\sigma(h, \mu)\left(\left\|\rho^{-1}(1+|\log \rho|)^{-1} u ; L_{2}(\widetilde{\Xi}(h))\right\|^{2}+\left\|u ; H^{1}(\widetilde{\Xi}(h))\right\|^{2}\right) \\
+\mu \sum_{j=1}^{J}\left|u^{j} ; \widetilde{\Omega}_{h}^{j}\right|_{j}^{2} \leqslant c \mathscr{E}_{\mu}(u ; \widetilde{G}(h)) .
\end{gathered}
$$

Здесъ $\mathscr{E}_{\mu}(u ; \widetilde{G}(h))$ - квадратичная форма (4.3) после замен $\Xi(h) \mapsto \widetilde{\Xi}(h)$ и $\Omega_{h}^{j} \mapsto \widetilde{\Omega}_{h}^{j}, \rho(x)=\min \left\{\operatorname{dist}\left(x, \Upsilon^{j}\right) \mid j=1, \ldots, J\right\}-$ весовой множитель на $\Xi$ $u \mathbf{|} u^{j} ; \widetilde{\Omega}_{h}^{j} \boldsymbol{\|}_{j}$ - норма $(2.42)$, в которой $\Omega_{h}=\widetilde{\Omega}_{h}^{j}$. Множитель $\sigma(h, \mu)$ определяется следующим образом:

1) $\sigma(h, \mu)=h^{2} \min \{1, h \mu\}$, если линии $\Upsilon^{j}=\omega^{j} \cap \partial \Xi, j=1, \ldots, J$, лежат на общей прямой;

2) $\sigma(h, \mu)=\min \left\{(1+|\log h|)^{-2}, h^{3} \mu\right\}$, если выполнено условие треуголъникa $\left.2^{\circ}\right)$;

3) $\sigma(h, \mu)=\min \left\{(1+|\log h|)^{-2}, h \mu\right\}$, если выполнено условие $\left.1^{\circ}\right)$ заполнения пространства.

Поясним геометрическое требование в теореме $4.1,3)$.

Лемма 4.2. 1) Если дуги $\Upsilon^{1}, \ldots, \Upsilon^{J}$ содержатся в одной плоскости $\Pi=$ $\left\{x: x_{3}=0\right\}$, то линейная оболочка из условия $\left.1^{\circ}\right)$ трехмерна и ее ортогональное дополнение натянуто на столбци $\mathbf{e}_{l}=\left(\delta_{1, l}, \ldots, \delta_{6, l}\right)^{\top}$, где $l=3,4,5$, a $\delta_{k, l}$ - символ Кронекера. Жесткие смещения $d(x) \mathbf{e}_{l}$ соответствуют параллельному переносу плоскости П и вращениям вокруг прямых, лежащих в этой плоскости.

2) Если дуги $\Upsilon^{1}, \ldots, \Upsilon^{J}$ размещены на параллельных плоскостях $\Pi^{j}=\{x$ : $\left.x_{3}=x_{3}^{j}\right\}$ и $x_{3}^{1} \neq x_{3}^{k}$ при каком-то номере $k$, то линейная оболочка из условия $1^{\circ}$ ) пятимерна и ей ортогонален столбеи, $\mathbf{e}_{3}$, отвечающий параллельному переносу плоскостей.

3) Если дуги $\Upsilon^{1}, \ldots, \Upsilon^{J}$ расположены в плоскостях $\Pi^{j}$, пересекающихся по оси аппликат $\Lambda=\left\{x: x_{1}=x_{2}=0\right\}$, но $\Upsilon^{j} \backslash \Lambda \neq \varnothing$ по крайней мере для двух различных номеров $j$, то линейная оболочка из условия $1^{\circ}$ ) пятимерна и ей ортогонален столбец $\mathbf{e}_{6}$, отвечающий вращению вокруг прямой $\Lambda$.

4) Если среди $\Upsilon^{1}, \ldots, \Upsilon^{J}$ имеются три дуги на трех разных плоскостях $\Pi^{k}$, $\Pi^{l}, \Pi^{m}$, две из которьх, скажем $\Pi^{k}$ u $\Pi^{l}$, пересекаются и линия их пересечения не содержится в $\Pi^{m}$, а сами дуги не принадлежат целиком линиям пересечения отобранных плоскостей, то требование $1^{\circ}$ ) соблюдено.

Признаемся, что приведенная переформулировка условия $1^{\circ}$ ) запутанная, и далее сопроводим ее примером 4.1. Если дуга $\Upsilon^{j}$ не является отрезком прямой, то условие треугольника $2^{\circ}$ ) заведомо выполнено. В частности, непараллельные цилиндры $\mathscr{C}_{h}^{m}$ и $\mathscr{C}_{h}^{p}$ можно выбрать внутри одного искривленного гнезда $g_{h}^{j}$ и получить неравенство Корна из теоремы $\left.4.1,2\right)$. Условие $1^{\circ}$ ), согласно лемме $4.2,1$ ), безразлично к искривленности дуг $\Upsilon^{1}, \ldots, \Upsilon^{J}$. 
4.3. Пластины, вставленные в глубокие прорези. В случае пластины (4.1), глубоко погруженной в тело $\Xi$, можно увеличить объем множества $\mathscr{C}_{h}^{j}$, а именно, поместить в гнезде $g_{h}^{j}=\Xi \cap \Omega_{h}^{j}$ круговой цилиндр с высотой $O(h)$ и радиусом основания $O(1)$. В результате все элементы аналогичной $(3.17)$ матрицы Грама оказываются величинами $O(h)$, а проверка очередных утверждений становится необременительной.

ТеОрема 4.2 [52]. Для поля $u \in \stackrel{\circ}{H}^{1}(G(h) ; \Gamma(h))^{3}$ справедливо неравенство Корна

$$
\sigma(h, \mu)\left\|u ; H^{1}(\Xi)\right\|^{2}+\mu \sum_{j=1}^{J} \mid u^{j} ; \Omega_{h}^{j} \mathbf{|}_{j}^{2} \leqslant c \mathscr{E}_{\mu}(u ; G(h)),
$$

где $\mid u^{j} ; \Omega_{h}^{j} \mathbf{I}_{j}-$ норма (2.42) при $\rho_{h}\left(y^{j}\right)=h+\operatorname{dist}\left(y^{j}, \Upsilon^{j}\right)$, а множитель $\sigma(h, \mu)$ определяется следующим образом:

1) если среди плоскостей $\Pi^{1}, \ldots, \Pi^{J}$ найдутся три разные плоскости, две из которых пересекаются, а третъя не содержит линию пересечения иеликом, $\operatorname{mo} \sigma(h, \mu)=\min \{1, h \mu\}$;

2) если множества $\omega^{1}, \ldots, \omega^{J}$ лежат на параллельных плоскостях или на плоскостях, пересекающихся по общей прямой, $\operatorname{mo} \sigma(h, \mu)=\min \left\{1, h^{3} \mu\right\}$.

Заметим, что даже при одной пронизывающей пластине условие треугольника всегда выполнено: на пересечении $\Xi \cap \omega^{j}$ помещается треугольник положительной площади. Именно поэтому в формулировке теоремы 4.2 не возникает какая-либо третья геометрическая ситуация.

Следующее утверждение показывает, в частности, что теорема $4.2,1$ ) опирается на условие заполнения пространства $\mathbb{R}^{6}$.

Лемма 4.3. 1) Если $\Pi^{1}=\cdots=\Pi^{J}=\left\{x: x_{3}=0\right\}$, то подпространство

$$
\mathfrak{L}\left\{d(x)^{\top} e_{i}^{j} \mid x \in \Xi \cap \omega^{j}, i=1,2, j=1, \ldots, J\right\} \subset \mathbb{R}^{6}
$$

трехмерно и его ортогональное дополнение натянуто на столбцы $\mathbf{e}_{3} u \mathbf{e}_{4}, \mathbf{e}_{5}$.

2) Если $J>1$ и $\Pi^{1}=\left\{x: x_{3}=0\right\}$, а остальные плоскости $\Pi^{2}, \ldots, \Pi^{J}$ nараллельны $\Pi^{1}$ (пересекаятся $с \Pi^{1}$ по оси абсцисс), но хотя бы одна из них не совпадает с $\Pi^{1}$, то подпространство (4.9) пятимерно и ортогонально столбиу $\mathbf{e}_{3}$ (столбиу $\left.\mathbf{e}_{4}\right)$.

3) Если выполнены условия теоремы 4.2, 1), то $\mathfrak{L}\{\ldots\}=\mathbb{R}^{6}$.

4.4. Обсуждение. Соотношения между жесткостями элементов конструкций и параметром $h$, различаемые в теоремах 4.1 и 4.2, обусловлены следующими связями для множителей $\sigma(h, \mu)$ в неравенствах (4.7) и (4.8):

$$
\begin{aligned}
\min \left\{(1+|\log h|)^{-2}, h \mu\right\} & \geqslant \min \left\{(1+|\log h|)^{-2}, h^{3} \mu\right\} \\
& \geqslant h^{2} \min \left\{(1+|\log h|)^{-2}, h \mu\right\}, \\
\min \{1, h \mu\} & \geqslant \min \left\{1, h^{3} \mu\right\} .
\end{aligned}
$$

Порядки жесткостей пластины на растяжение и изгиб как раз равны $O(h \mu)$ и $O\left(h^{3} \mu\right)$ (см., например, [15]). В то же время формулы $(3.22)$ и (3.25) для сочленения тела со стержнями включают жесткости стержня на растяжение $h^{2} \mu$ и изгиб $h^{4} \mu$. 
Сравнивая список $\left(4.10_{1}\right)$ со списками (3.22) и (3.25), видим, что в первом стоят величины, не меньшие величин во втором или в третьем. Это наблюдение согласуется с житейски очевидным фактом: чем шире шип и чем глубже паз, тем прочнее соединение и тем лучше оно передает усилия с одного элемента конструкции на другой.

Еще одно объяснение подмеченному правилу находим при анализе хода доказательств обсуждаемых неравенств Корна: по мере возрастания множителя $\sigma(h, \mu)$ удавалось поместить в гнезда $g_{h}^{j}$ цилиндры $\mathscr{C}_{h}^{m}$ все большего объема, а именно, $O\left(h^{3}\right)$ в пп. 3.2, 3.3, $O\left(h^{2}\right)$ в пп. 3.4, 4.2, и, наконец, $O(h)$ в предыдущем пункте. С увеличением размера цилиндра $\mathscr{C}_{h}^{m}$ растут (во всяком случае не убывают) собственные числа матрицы $\mathbf{d}\left(\mathscr{C}_{h}^{m}\right)$, а значит, улучшаются оценки компонент столбца $b$, которые и определяют множитель $\sigma(h, \mu)$ (ср. замечание 2.7).

Для стержней, пронизывающих тело, и пластин, вставленных в неглубокие пазы, рассматривались одинаковые стержни-штыри $\mathscr{C}_{h}^{m}$. Для них величина $\sigma(h, \mu)$ из соответствующего неравенства Корна в первую очередь обусловлена строением анизотропной нормы (2.52) для стержня или (2.42) для пластины. В норме (2.52) "слабыми" оказываются два поперечных направления, а в норме (2.42) - только одно. Кроме того, благодаря возможности применить следствие (2.45) неравенства Харди (замечание 2.5) в выражениях $\left(4.10_{1}\right)$ при относительной жесткости $\mu$ возникает дополнительный по сравнению с $(3.25)$ множитель $h^{-1}$.

Приведем несколько примеров, подтверждающих точность проверенных неравенств Корна и сформулированных геометрических требований.

ПримеР 4.1. 1) Скребок. Пусть $\Xi$ - цилиндр $\left\{x: x_{1}^{2}+\left(x_{2}-1\right)^{2}<1,\left|x_{3}\right|<1\right\}$, а $\Omega_{h}^{1}$ и $\widetilde{\Omega}_{h}^{1}$ - параллелепипеды $\left\{x:\left|x_{1}\right|<h, x_{2} \in(-1,1),\left|x_{3}\right|<1\right\}$ и $\{x$ : $\left.\left|x_{1}\right|<h, x_{2} \in(-h, 1),\left|x_{3}\right|<1\right\}$ соответственно (см. рис. 7). При жесткой пластине $\widetilde{\Omega}_{h}^{1}$, т. е. при $h \mu>1$, асимптотическую точность неравенства (4.7) с множителем $\sigma(h, \mu)=h^{2}$ подтверждает поле $u^{\theta}$ из замечания 2.3. Оно же демонстрирует необходимость дополнительного условия в теореме $4.1,2)$. Если пластина $\widetilde{\Omega}_{h}^{1}$ мягкая и $h \mu \leqslant 1$, то годится поле

$$
v^{\theta}(x)=\left(-x_{2} \chi\left(x_{2}\right), x_{1} \chi\left(x_{2}\right)+x_{1} \frac{\partial}{\partial x_{2}}\left(x_{2} \chi\left(x_{2}\right)\right), 0\right)^{\top},
$$

которое обращается в нуль на грани $\Gamma_{h}^{1}=\left\{x:\left|x_{1}\right|<h, x_{2}=1,\left|x_{3}\right|<1\right\}$ параллелепипеда $\widetilde{\Omega}_{h}^{1}$. Только деформация $\varepsilon_{22}\left(v^{\theta} ; x\right)=x_{1}\left(2 \partial_{x_{2}}+x_{2} \partial_{x_{2}^{2}}^{2}\right) \chi\left(x_{2}\right)$ отлична от нуля, а значит, левая и правая части неравенства $(4.7)$ при $\sigma(h, \mu)=h^{3} \mu$ составляют $O\left(h^{3} \mu\right)$. То же поле (4.11) указывает нужный пример в случае мягкого или не очень жесткого $\left(h^{3} \mu \leqslant 1\right)$ "лезвия" $\Omega_{h}^{1}$, глубоко посаженного в "рукоятку" $\Xi$ (теорема 4.2, 2)). При очень жестком $\left(h^{3} \mu>1\right)$ лезвии достаточно подставить в неравенство (4.8) любую нетривиальную вектор-функцию, обращающуюся в нуль вне окрестности множества $\overline{\omega^{1}}$.

2) Голова в кепке. Пусть $\Xi=\mathbb{B}_{1}(0)$ - шар,

$$
\Omega_{h}^{1}=\left\{x: x_{1} \in(1 / 2,2),\left|x_{2}\right|<1 / 2,\left|x_{3}-1 / 2\right|<h\right\}
$$




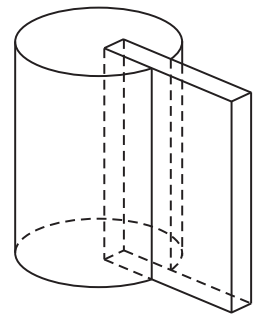

Рис. 7. Скребок

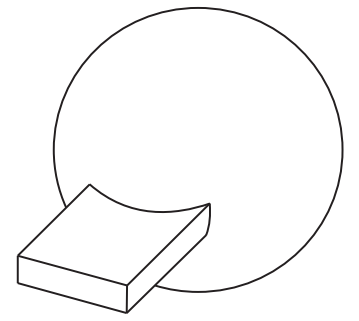

Рис. 8. Голова в кепке

и $\widetilde{\Omega}_{h}^{1}=\left\{x \in \Omega_{h}^{1}:\left|x^{\prime}\right|=x_{1}^{2}+x_{2}^{2}>-h+3 / 4\right\}$ (см. рис. 8). Это сочленение удовлетворяет условиям теорем $4.1,2)$ и $4.2,2)$. Если $h^{3} \mu<(1+|\log h|)^{-2}$, то при всяком $b \in \mathbb{R}^{6}$ поле

$$
d(x) b\left(1-\chi_{-}\left((\log h)^{-1} \log || x^{\prime}\left|-\frac{\sqrt{3}}{2}\right|\right) \chi_{-}\left((\log h)^{-1} \log \left|x_{3}-\frac{1}{2}\right|\right)\right), \quad x \in \Xi
$$

(ср. с замечанием $2.3,1)$ ), продолженное нулем на множество $\Omega_{h}^{1} \backslash \Xi$, превращает обе части неравенства (4.7) при $\sigma(h, \mu)=(1+|\log h|)^{-1}$ в ограниченные величины $O\left(h^{0}\right)$.

Асимптотическую точность неравенства (4.8) при $\sigma(h, \mu)=1$ подтверждает любое поле $u$ с носителем $\operatorname{supp} u \subset \Xi \backslash \overline{\Omega_{h}^{i}}$ при всех $h \in\left(0, h_{0}\right]$.

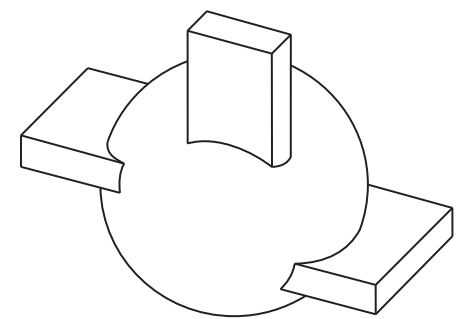

Рис. 9. Треух с приподнятым задком

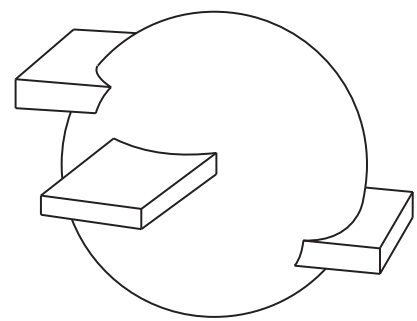

Рис. 10. Треух с опущенным задком

3) Голова в треухе. Пусть $\Xi=\mathbb{B}_{1}(0)$,

$$
\begin{gathered}
\Omega_{h}^{1}=\left\{x:\left|x_{1}-\frac{1}{2}\right|<h,\left|x_{2}\right|<\frac{1}{2}, x_{3} \in\left(\frac{1}{2}, 2\right)\right\}, \\
\Omega_{h}^{1+p}=\left\{x:\left|x_{1}\right|<\frac{1}{2},(-1)^{p} x_{2} \in\left(\frac{1}{2}, 2\right),\left|x_{3}-\frac{(-1)^{p}}{2}\right|<h\right\}, \quad p=1,2,
\end{gathered}
$$

а пластины $\widetilde{\Omega}_{h}^{1}$ и $\widetilde{\Omega}_{h}^{1+p}$ выделены дополнительными условиями $x_{2}^{2}+x_{3}^{2}>-h+3 / 4$ и $x_{2}^{2}+x_{2}^{2}>-h+15 / 16$ (см. рис. 9, где голова смотрит в сторону от читателя, а задок треуха приподнят). Если на гранях параллелепипедов (4.13), которые не имеют общих точек с шаром $\Xi$, назначить условия (2.13), то окажутся выполненными предположения, сформулированные в теоремах 4.1, 3) и 4.2, 1), - 
поэтому считаем пластины мягкими, т. е. $h \mu \leqslant(1+|\log h|)^{-2}$ и $h \mu \leqslant 1$ соответственно. Поле смещений, определенное формулами

$$
\begin{array}{rlrl}
u(x) & =e_{3}, & x \in \Xi, \\
u(x)=\chi\left(x_{3}\right) e_{3}, & x \in \Omega_{h}^{1}, \\
u(x)=\chi\left(\left|x^{\prime}\right|\right) e_{3}-\sum_{i=1}^{2} e_{i} \hat{z}_{p} \frac{\partial}{\partial x_{i}} \chi\left(\left|x^{\prime}\right|\right), & & x \in \Omega_{h}^{1+p},
\end{array}
$$

в которых $\hat{z}_{p}=x_{3}-(-1)^{p} / 2$, делает правые и левые части неравенств $(4.7)$ и (4.8) с множителем $\sigma(h, \mu)=h \mu$ равными $O(h \mu)$.

Для того чтобы убедиться в невозможности упомянутых неравенств при нарушении условия $1^{\circ}$ ), заменим пластину $\left(4.13_{1}\right)$ пластиной $(4.12)$ (ср. рис. 10 , на котором задок треуха опущен). Положив $\hat{z}_{1}=x_{3}$ и распространив определение $\left(4.14_{3}\right)$ поля $u$ на $\Omega^{1}(h)$, обнаруживаем, что

$$
\mathscr{E}_{\mu}(u ; G(h))=\mathscr{E}_{\mu}(u ; \widetilde{G}(h))=O\left(h^{3} \mu\right),
$$

т. е. множитель $\sigma(h, \mu)=h \mu$ недопустим.

Тот же эффект наблюдается, если надеть треух аккуратно, расположив его уши в одной плоскости: в формуле $\left(4.13_{2}\right)$ убираем вычитаемое $(-1)^{p} / 2$, но формулу (4.131) сохраняем (вообще же, положение задка теперь безразлично). Три пластины имеют общую линию пересечения, по леммам 4.2, 3) и 4.3, 2) условие $1^{\circ}$ ) нарушено, а невозможность утверждений теорем $\left.4.1,3\right)$ и $4.2,1$ ) устанавливается при помощи такого поля:

$$
\begin{array}{lll}
u(x)=-e_{1} x_{3}-e_{3}\left(x_{1}-\frac{1}{2}\right), & x \in \Xi, & \\
u(x)=-e_{1} x_{3} \chi\left(x_{3}\right)+e_{3}\left(x_{1}-\frac{1}{2}\right) \frac{\partial}{\partial x_{3}} x_{3} \chi\left(x_{3}\right), & x \in \Omega_{h}^{1}, & \\
u(x)=e_{3}\left(x_{1}-\frac{1}{2}\right) \chi\left(x_{1}\right)-e_{1} x_{3} \frac{\partial}{\partial x_{1}}\left(x_{1}-\frac{1}{2}\right) \chi\left(x_{1}\right), & x \in \Omega_{h}^{1+p}, \quad p=1,2 .
\end{array}
$$

4.5. Незакрепленные пластины. Выступающие из тела края некоторых из пластин $\Omega^{j}(h)$ и $\Omega_{h}^{j}, j=1, \ldots, J$, могут быть свободными, а условия $\left(2.18_{1}\right)$ при соответствующих индексах $j$ - отсутствовать. В этом случае объекты с такими индексами $j$ изымаются из требований $\left.1^{\circ}\right), 2^{\circ}$ ) и формулы (4.9). В очередном утверждении содержатся неравенства Корна, обслуживающие незакрепленные пластины, вытекающие из неравенств Харди (2.27), (2.44) и теорем 2.5, 2.7 .

Теорема 4.3. Для поля $u \in H^{1}\left(\Xi \cup \Omega_{h}^{j}\right)^{3}$ справедливы неравенства

$$
\begin{aligned}
& \left|u^{j} ; \Omega_{h}^{j}\right|^{2} \leqslant c\left(\mathscr{E}_{1}\left(u ; \Omega_{h}^{j}\right)+h\left\|u ; H^{1}(\Xi)\right\|^{2}\right), \\
& \left|u^{j} ; \Omega_{h}^{j}\right|^{2} \leqslant c\left(\mathscr{E}_{1}\left(u ; \Omega^{j}(h)\right)+(1+|\log h|)^{2}\left\|u ; H^{1}(\Xi)\right\|^{2}\right) .
\end{aligned}
$$

Асимптотический анализ сочленений массивных тел с тонкими пластинами проводился в работах [53]-[56], книге [57] и многих других публикациях. 


\section{§ 5. Неравенства Корна для сочленения пластины со стержнями}

5.1. Геометрия сочленений. Пусть $\omega^{0}, \omega^{1}, \ldots, \omega^{J}$ - области на плоскости $\mathbb{R}^{2}$, имеющие кусочно гладкие (можно липшицевы) границы и компактные замыкания. Пусть $\Omega_{h}^{0}$ - цилиндрическая (для простоты) пластина (2.33), причем $\omega=\omega^{0}$ и $H_{ \pm}\left(x^{\prime}\right)=1 / 2$. Пусть еще $Q_{h}^{1}, \ldots, Q_{h}^{J}$ - цилиндры с осями $\Lambda^{1}, \ldots, \Lambda^{J}$, пересекающими множество $\omega^{0} \subset \mathbb{R}^{2} \times\{0\} \subset \mathbb{R}^{3}$,

$$
Q_{h}^{j}=\left\{x \in \mathbb{R}^{3}: \eta^{j}=\left(\eta_{1}^{j}, \eta_{2}^{j}\right):=h^{-1} y^{j} \in \omega^{j}, z^{j} \in \mathbb{R}^{3}\right\}
$$

здесь $j=1, \ldots, J$ и $x^{j}=\left(y^{j}, z^{j}\right)$ - декартовы координаты, причем $y^{j}=\left(y_{1}^{j}, y_{2}^{j}\right)-$ координаты в плоскости, перпендикулярной оси $\Lambda^{j}$. Не ограничивая общности, предполагаем, что сечение $\omega^{j}$ содержит начало координат $y^{j}=0$. Систему координат $x=(y, z)$ (без верхнего индекса) считаем привязанной к пластине $\Omega_{h}^{0}$.

При описании стержней следует различать два случая: стержень $G_{h}^{j}$ вставлен в гнездо $g_{h}^{j}=G_{h}^{j} \cap \Omega_{h}^{0}$ или приклеен к пластине вдоль поверхности $\mathscr{T}_{h}^{j} \subset \partial \Omega_{h}^{0}$. В первом случае отрезаем стержень $G_{h}^{j}$ от цилиндра (5.1) двумя плоскостями; торец, заглубленный в пластину, обозначаем $\omega_{h}^{j 1} \subset \Omega_{h}^{0}$, а другой $-\omega_{h}^{j 0}$. Под $\omega_{h}^{j}(t)$ подразумевается сечение цилиндра $Q_{h}^{j}$, не обязательно перпендикулярное его оси $\Lambda^{j}$, но параллельное торцу $\omega_{h}^{j 1}$ и проходящее через точку $z^{j}=t,-$ координату $z^{j}$ и длину $l_{j}>0$ выбираем так, чтобы $\omega_{h}^{j 1}=\omega_{h}^{j}(0)$ и $\omega_{h}^{j 0}=\omega_{h}^{j}\left(l_{j}\right)$. Стержни защемлены вдоль поверхностей $\Gamma_{h}^{j 0}=\omega_{h}^{j 0}$.

Во втором случае стержень реально занимает объем $G_{h}^{j} \backslash \overline{\Omega_{h}^{0}}$ и отсекается от $Q_{h}^{j}$ поверхностью $\mathscr{T}_{h}^{j}=G_{h}^{j} \cap \partial \Omega_{h}^{0}$. Далее рассматриваем только гнездовое крепление стержней - изменения, необходимые для перехода к поверхностному приклеиванию, уже обсуждались в $§ 2$. Лемма 5.1 показывает, как нужно изменить последующие утверждения.

ЛЕмма 5.1 ([40]; ср. с предложением 2.5). Выполнено неравенство

$$
\min \{1, \mu\}\left\|u ; G_{h}^{j} \cap \Omega_{h}^{0} \boldsymbol{|}^{2}+\mu \mid u ; G_{h}^{j} \backslash \Omega_{h}^{0}\right\|^{2} \leqslant c\left(\mathscr{E}\left(u ; G_{h}^{j} \cap \Omega_{h}^{0}\right)+\mu \mathscr{E}\left(u ; G_{h}^{j} \backslash \overline{\Omega_{h}^{0}}\right)\right),
$$

в котором |...| - норма (2.52) при $\rho_{h}=1$.

Сочленение пластины и стержней - область $\Xi(h)$, замыкание которой имеет вид $\overline{\Xi(h)}=\overline{\Omega_{h}^{0}} \cup \overline{G_{h}^{1}} \cup \cdots \cup \overline{G_{h}^{J}}$. Сочленение закреплено по множеству $\Gamma_{h}^{0}=$ $\Gamma_{h}^{10} \cup \cdots \cup \Gamma_{h}^{J 0}$, т. е. выполнены условия $\left(2.18_{1}\right)$ при $j=1, \ldots, J$. Через $\mu$ обозначим относительную жесткость стержней, т. е. аналогично (3.2) и (2.56) определим квадратичную форму

$$
\mathscr{E}_{\mu}(u ; \Xi(h))=\left\|D\left(\nabla_{x}\right) u^{0} ; L_{2}\left(\Omega^{0}(h)\right)\right\|^{2}+\mu \sum_{j=1}^{J}\left\|D\left(\nabla_{x}\right) u ; L_{2}\left(G_{h}^{j}\right)\right\|^{2},
$$

где $\Omega^{0}(h)=\Omega_{h}^{0} \backslash\left(\overline{G_{h}^{1}} \cup \cdots \cup \overline{G_{h}^{J}}\right)$. Форма элементов сочленения (цилиндрические тела) упрощена сознательно, так как в свете предшествующих результатов всевозможные обобщения очевидны. То же касается и формы гнезд. Имея в виду проблему, сформулированную в книге [58; с. 293] более тридцати лет назад, называем пластину $\Omega_{h}^{0}$ столешней, а стержни $G_{h}^{j}-$ ножками вне зависимости от их положения относительно столешни. 
5.2. Один или два стержня. Теоремы $2.9,2.10$ доставляют оценки полей смещений на ножках, а следствие 2.2 - на их частях, вставленных в гнезда $g_{h}^{j}$. Выбрав малые множества $\mathscr{C}_{h}^{j} \subset g_{h}^{j}$, можно модифицировать, зачастую в значительной степени, рассуждения из $\S 3$, и свести вопрос к оценке компонент столбца $b^{0} \in \mathbb{R}^{6}$ в разложении поля $u$ на столешне

$$
u(x)=u^{\perp}(x)+d(x) b^{0} .
$$

Составляющая $u^{\perp}$, подчиненная условию ортогональности $F^{* h}\left(u^{\perp}\right)=0 \in \mathbb{R}^{6}$ с функционалом (2.40), обрабатывается при помощи теоремы 2.5 и следствия 2.1. Набор алгебраических связей для компонент столбца $b^{0}$ зависит от количества и расположения стержней, которые и предопределяют весовые множители в норме ||$u ;\left.\Omega_{h}^{0}\right|_{\beta}$, заданной формулой (2.48) на столешне, а появление логарифмов обусловлено неравенством Харди (2.26).

Теорема 5.1 [40]. Для поля $u \in \stackrel{\circ}{H^{1}}(\Xi(h) ; \Gamma(h))^{3}$ справедливо неравенство Корна

$$
\mathbf{\|} u ; \Omega^{0}(h) \boldsymbol{|}_{\beta}^{2}+\mu \sum_{j=1}^{J} \mid u^{j} ; G_{h}^{j} \mathbf{|}_{j}^{2} \leqslant c \mathscr{E}_{\mu}(u ; \Xi(h)),
$$

где $\mathbf{I} ; G_{h}^{j} \mathbf{I}_{j}-$ норма (2.52) при $\rho_{h}\left(z^{j}\right)=h+\left|z^{j}-l_{j}\right|$, а множители $\beta^{\prime}(h, \mu)$ и $\beta_{z}(h, \mu)$ в норме (2.48), первой в левой части (5.4), определяются следующим образом:

1) если $J=1$ или $P^{1}=\cdots=P^{J}, \operatorname{mo} \beta^{\prime}(h, \mu)=h^{2} \min \{1, h \mu\}$ u $\beta_{z}(h, \mu)=$ $\min \left\{(1+|\log h|)^{-2}, h \mu\right\}$;

2) если все точки $P^{1}, \ldots, P^{J}$ лежсат на одной прямой и $P^{1} \neq P^{2}$, то $\beta^{\prime}(h, \mu)=\min \left\{(1+|\log h|)^{-2}, h^{3} \mu\right\} u \beta_{z}(h, \mu)=\min \left\{(1+|\log h|)^{-2}, h \mu\right\}$.

Опишем конкретные конструкции, подтверждающие асимптотическую точность неравенства Корна из теоремы 5.1.

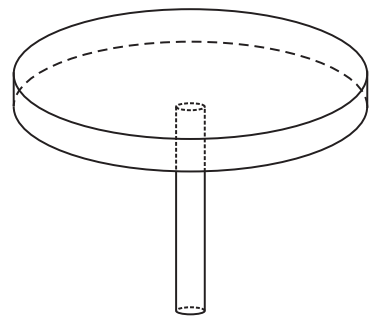

Рис. 11. Гриб

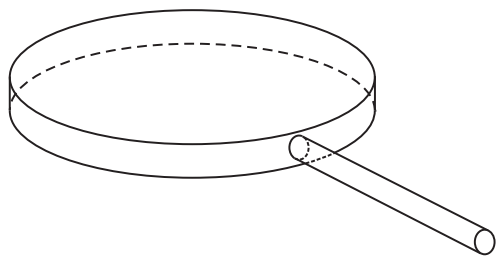

Рис. 12. Опахало

ПримеР 5.1. 1) Гриб и опахало. Пусть

$$
\Omega_{h}^{0}=\{x:|y|<1,|z|<h / 2\}, \quad G_{h}^{1}=\{x:|y|<h, z \in(-2,0)\}
$$

или

$$
\begin{aligned}
& \Omega_{h}^{0}=\left\{x:\left|y_{1}+1\right|^{2}+y_{2}^{2}<1,|z|<h / 2\right\}, \\
& G_{h}^{1}=\left\{x:\left|y_{2}\right|^{2}+|z|^{2}<h^{2}, y_{1} \in(-h, 2)\right\} .
\end{aligned}
$$


В обоих случаях $P^{1}=\mathscr{O}$. Соответствующие сочленения изображены на рис. 11 и 12. Если ножка гриба или ручка опахала жесткие, т. е. $h \mu \geqslant(1+|\log h|)^{-2}$, правильность определения множителей $\beta^{\prime}(h, \mu)$ и $\beta_{z}(h, \mu)$ в теореме $\left.5.1,1\right)$ подтверждается рассмотрением умноженных на срезающие функции векторов поворота

$$
\begin{aligned}
u^{i}(x) & =\left(z e_{i}-y_{i} e_{3}\right)\left(1-\chi\left(h^{-1}|x|\right)\right), \quad i=1,2, \\
u^{3}(x) & =\left(y_{1} e_{2}-y_{2} e_{1}\right)\left(1-\chi\left(h^{-1}|x|\right)\right)
\end{aligned}
$$

(ср. с замечанием 2.3). Для них аналогично выкладке (2.23) получаем

$$
\begin{gathered}
\mathscr{E}_{\mu}\left(u^{p} ; \Xi(h)\right) \leqslant c h^{3}, \quad p=1,2,3, \\
\left\|\left|u^{i} ; \Omega^{0}(h)\right| \mathbf{|}^{2} \geqslant c h^{3}, \quad \quad\right\| u^{3} ; \Omega^{0}(h) \|_{\beta}^{2}=\beta^{\prime}(h, \mu) \mid u^{3} ; \Omega^{0}(h) \boldsymbol{|}^{2} \geqslant c \beta^{\prime}(h, \mu) h .
\end{gathered}
$$

Если же стержни мягкие и $h \mu<(1+|\log h|)^{-2}$, то положим

$$
\begin{array}{ll}
u^{3}(x)=\left(y_{1} e_{2}-y_{2} e_{1}\right), & x \in \Omega_{h}, \\
u^{3}(x)=\left(y_{1} e_{2}-y_{2} e_{1}\right) \chi_{-}(z), & x \in G_{h}^{1},
\end{array}
$$

в случае (5.5) и

$$
\begin{gathered}
u^{i}(x)=\left(z e_{i}-y_{i} e_{3}\right), \quad i=1,2, \quad x \in \Omega_{h}, \\
u^{1}(x)=y_{1} e_{3} \chi_{-}\left(y_{1}\right)-z e_{1} \frac{\partial}{\partial y_{1}} y_{1} \chi_{-}\left(y_{1}\right), \quad u^{2}(x)=\left(z e_{2}-y_{2} e_{3}\right) \chi_{-}\left(y_{1}\right), \quad x \in G_{h}^{1},
\end{gathered}
$$

в случае (5.6). Здесь $\chi_{-}$- срезающая функция из примера 2.3. Соотношения $\left(5.8_{2}\right)$ остаются верными, но теперь $\mathscr{E}_{\mu}\left(u^{p} ; \Xi(h)\right) \leqslant c \mu h^{4}$.

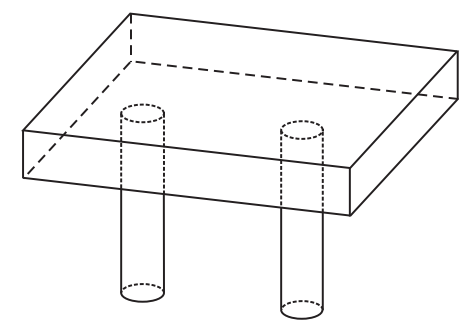

Рис. 13. Садовая скамейка

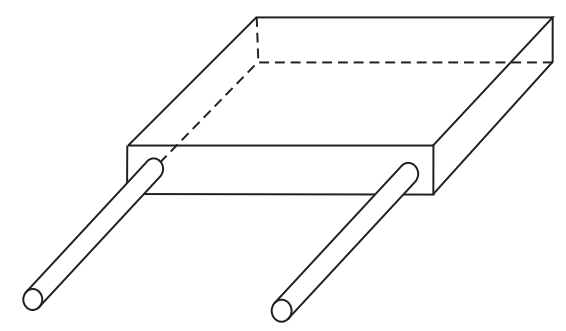

Рис. 14. Волокуши

2) Садовая скамейка и волокуши. Сочленения, в которых

$$
\begin{gathered}
\Omega_{h}^{0}=\left\{x:\left|y_{1}\right|<1 / 2,\left|y_{2}\right|<2,|z|<h / 2\right\}, \\
G_{h}^{j}=\left\{x:\left|y_{1}\right|^{2}+\left|y_{2}-(-1)^{j}\right|^{2} \leqslant h^{2}, z \in(-1,0)\right\}, \\
P^{j}=\left(0,(-1)^{j}, 0\right)^{\top}, \quad j=1,2,
\end{gathered}
$$


или

$$
\begin{gathered}
\Omega_{h}^{0}=\left\{x: y_{1} \in(-1,0),\left|y_{2}\right|<1\right\}, \\
G_{h}^{j}=\left\{x: y_{1} \in(-h, 2),\left|y_{2}-(-1)^{j} \frac{1}{2}\right|^{2}+|z|^{2}<h^{2}\right\}, \\
P^{j}=\left(0,(-1)^{j} \frac{1}{2}, 0\right)^{\top}, \quad j=1,2,
\end{gathered}
$$

изображены на рис. 13 и 14. Убедимся в том, что множитель $\beta^{\prime}(h, \mu)$, указанный в теореме $5.1,2)$ при $(1+|\log h|)^{-2} \leqslant h^{3} \mu$, т. е. для "очень жестких" стержней - ножек и оглоблей соответственно, оказывается точным без учета логарифмов (ср. замечание 2.3). В обоих случаях годится поле, заданное формулой

$$
u^{2}(x)=\left(z e_{1}-y_{1} e_{3}\right)\left(1-X_{h}(x)\right)
$$

на пластине $\Omega_{h}^{0}$ и продолженное нулем на стержни $G_{h}^{1}$ и $G_{h}^{2} ;$ при этом $X_{h}-$ cрезающая функция, равная нулю внутри $c h$-окрестностей и единице вне $C h$-окрестностей точек $P^{1}$ и $P^{2}$. Нужное заключение вытекает из соотношений $\mathscr{E}_{\mu}\left(u^{2} ; \Xi(h)\right) \leqslant c h^{3}, \mathbf{I} \mid u^{2} ; \Omega^{0}(h) \boldsymbol{|}_{\beta}^{2} \geqslant c \beta^{\prime}(h, \mu) h^{3}$.

$3)$ Вектор-функции (5.10) и (5.72) подходят для проверки правильности выбора множителей $\beta^{\prime}(h)$ и $\beta_{z}(h)$ в неравенстве Корна $(2.47)$ для пластины, защемленной по малым зонам.

Приведенные в примерах $5.1,1)$ и 2) конкретные векторы смещений демонстрируют, что добавление-в-ряд ножек у скамейки и оглоблей у волокуш не увеличивает весовые множители $\beta^{\prime}(h, \mu)$ и $\beta_{z}(h, \mu)$, а значит, не усиливает конструкцию - впрочем, предсказать это было нетрудно. В следующих трех пунктах будет установлено, что удачное размещение дополнительных стержней позволяет все-таки улучшить норму ||$\cdot ; \Omega^{0}(h) \mid \mathbf{I}_{\beta}$.

5.3. Шесть стержней. Пусть $J \geqslant 6$, а $\nu^{j}$ - единичный вектор оси $\Lambda^{j}$ цилиндра (5.1). Примем требование $1^{\circ}$ ) заполнения пространства $\mathbb{R}^{6}$, уже возникавшее в п. 3.2. Подчеркнем, что ограничение $P^{1} \neq P^{2}$ из теоремы $\left.5.1,2\right)$ снято и среди точек $P^{1}, \ldots, P^{J}$ могут быть одинаковые (допускаются конструкции, фрагментами которых служат “треноги” или “ко́злы”).

Теорема 5.2 [40]. Пусть выполнено условие $\left.1^{\circ}\right)$ из n. 3.2. Тогда для поля $u \in \stackrel{\circ}{H^{1}}(\Xi(h) ; \Gamma(h))^{3}$ справедливо неравенство Корна

$$
\mathbf{\|}\left|u ; \Omega^{0}(h)\right| \mathbf{|}_{\beta}^{2}+\mu \sum_{j=1}^{J}\left|u^{j} ; G_{h}^{j}\right|_{j}^{2} \leqslant c \mathscr{E}_{\mu}(u ; \Xi(h)),
$$

где $\mathscr{E}_{\mu}(u ; G(h))$ - квадратичная форма $(5.2), \mathbf{|} \cdot ; G_{h}^{j} \mathbf{I}_{j}-$ норма $(2.52)$ с $\rho_{h}\left(z^{j}\right)=$ $h+\left|z^{j}-l_{j}\right|$, а норма (2.48), первая в левой части (5.11), имеет множители $\beta^{\prime}(h, \mu)=\min \left\{(1+|\log h|)^{-2}, h^{3} \mu\right\} u \beta_{z}(h, \mu)=\min \left\{(1+|\log h|)^{-2}, h^{-1} \mu\right\}$.

По сравнению с теоремой $5.1,2)$ не произошло увеличения множителя $\beta^{\prime}(h, \mu)$, однако теперь множитель $\beta_{z}(h, \mu)$ оказывается равным $(1+|\log h|)^{-2}$ даже в случае "не очень мягких" стержней, т. е. при $\mu \geqslant h(1+|\log h|)^{-2}$. 
ПримеР 5.2. Таракан. Пусть $\Omega_{h}^{0}$ - эллиптическая в плане пластина $\{x$ : $\left.y_{1}^{2}+4 y_{2}^{2}<9,|z|<h / 2\right\}$, к которой снизу присоединены шесть тонких стержней с атрибутами (3.3) (см. рис. 15). Как и в примере 3.1, условие $1^{\circ}$ ) выполнено.

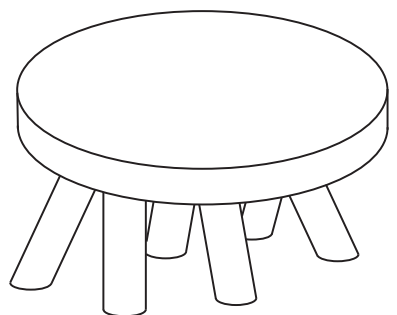

Рис. 15. Таракан

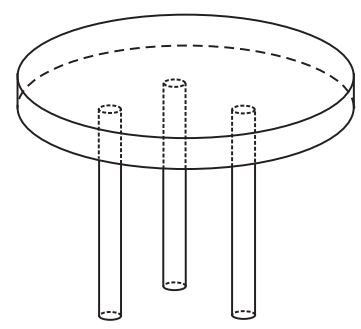

Рис. 16. Журнальный столик

5.4. Три вертикальных стержня. Конкретизируя положение осей $\Lambda^{j}$, можно уменьшить количество стержней при сохранении утверждения теоремы 5.2. Предположим, что

$\left.3^{\circ}\right)$ среди стержней $G_{h}^{1}, \ldots, G_{h}^{J}$ найдутся три, направленных вертикально и присоединенных $\kappa$ пластине в вершинах невырожденного треугольника.

ТеОРема 5.3 [40]. При условии $3^{\circ}$ ) утверждение теоремъ 5.2 сохраняет силу.

ПримеР 5.3. Журнальный столик. Пусть $\Xi(h)$ - сочленение, изображенное на рис. 16 (мы увеличили в примере $5.1,1$ ) число вертикальных цилиндрических ножек с закрепленными концами). Рассмотрим поля смещений $u^{i}(x)=$ $e_{i} \chi(z)-e_{3} y_{i}^{j} \partial_{z} \chi(z), i=1,2, u^{3}(x)=e_{3} \chi(z)$, где $\chi-$ срезка из примера 2.2 . Нетрудно убедиться в том, что

$$
\begin{array}{ll}
\mid u^{i} ; \Omega^{0}(h) \mathbf{|}^{2} \geqslant c h, & \mathscr{E}_{\mu}\left(u^{i} ; \Xi(h)\right) \leqslant C \mu h^{4}, \quad i=1,2, \\
\mathbf{|} u^{3} ; \Omega^{0}(h) \mathbf{|}^{2} \geqslant c h^{3}, & \mathscr{E}_{\mu}\left(u^{3} ; \Xi(h)\right) \leqslant C \mu h^{2} .
\end{array}
$$

Эти соотношения указывают на правильность расстановки весовых множителей в анизотропной норме $\left|\cdot ; \Omega^{0}(h)\right| \mathbf{I}_{\beta}$ "при очень мягких стержнях", а именно при $\mu<h(1+|\log h|)^{-2}$. Поле $(5.9)$ из примера $\left.5.1,1\right)$ подтверждает, что для двух стержней теорема 5.3 для "очень мягких стержней" неверна, т. е. условие $3^{\circ}$ ) необходимо и достаточно. В связи с проблемой [58; с. 293], уже упоминавшейся в п. 5.1, укажем, что увеличение количества ножек у стола не влияет на весовые множители в норме (2.48).

5.5. Три горизонтальных стержня. Пусть оси стержней $G_{h}^{1}, \ldots, G_{h}^{I}$, где $I \leqslant J$, расположены в плоскости $\{x: z=0\}$. Введем аналог ограничения $\left.1^{\circ}\right)$ из п. 2.2 и $\left.1^{\circ}\right)$ из п. 3.2 :

$\left.4^{\circ}\right) \mathbb{R}^{3}=\mathfrak{L}\left\{d^{\sharp}\left(P^{j}\right)^{\top} \nu^{j \sharp} \mid j=1, \ldots, I\right\}$. 
Здесь $\nu^{j \sharp}=\left(\nu_{1}^{j}, \nu_{2}^{j}\right)^{\top}$ и аналогично $(2.8)$

$$
d^{\sharp}(y)=\left(\begin{array}{ccc}
1 & 0 & -\alpha y_{2} \\
0 & 2 & \alpha y_{1}
\end{array}\right), \quad \alpha=\frac{1}{\sqrt{2}} .
$$

Теперь за счет геометрического условия удается изменить в норме $(2.48)$ весовые множители при производных $\partial_{2} u_{1}, \partial_{1} u_{2}$ и продольных смещениях $u_{1}, u_{2}$.

ТеОрема 5.4 [40]. Если выполнено условие $\left.4^{\circ}\right)$, то для поля $u \in \stackrel{\circ}{H^{1}}(\Xi(h)$; $\Gamma(h))^{3}$ справедливо неравенство Корна (5.11) с множителями $\beta^{\prime}(h, \mu)=$ $\beta_{z}(h, \mu)=\min \left\{(1+|\log h|)^{-2}, h \mu\right\}$ в весовой норме ||$\cdot ; \Omega^{0}(h) \mid \mathbf{I}_{\beta}$.

Если стержни жесткие и $\mu \geqslant h^{-1}(1+|\log h|)^{-2}$, то норма, указанная теоремой 5.4, совпадает с нормой в теореме 2.8 , обслуживающей пластину, которая закреплена по двум малым множествам. Эта же норма лишь множителями $1+|\log h|$ и $\rho_{h}^{-1}$ отличается от нормы $(2.42)$, соответствующей пластине с полностью защемленной кромкой. Иными словами, трех жестких растяжек вполне достаточно для фиксации пластины.

ЗАмечАниЕ 5.1. Обращаем внимание на то, что условие в формуле $\left(2.49_{1}\right)$ из теоремы 2.8 равносильно следующему:

$$
\mathbb{R}^{3}=\mathfrak{L}\left\{d^{\sharp}\left(P^{j}\right)^{\top} a \mid a \in \mathbb{R}^{3}, j=1, \ldots, J\right\} .
$$

Иными словами, названное условие и условие $4^{\circ}$ ) находятся в таком же отношении, как условия $2^{\circ}$ ) и $1^{\circ}$ ) в п. 2.2. Линейная оболочка $\mathfrak{L}\{\ldots\}$ совпадает с пространством $\mathbb{R}^{3}$ при $J=2$ и $P^{1} \neq P^{2}$.

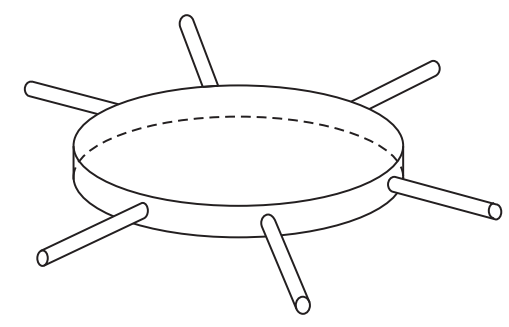

Рис. 17. Рулевое колесо

ПримеР 5.4. Рулевое колесо. Пусть $\Xi(h)$ - сочленение, изображенное на рис. 17, т. е. пластина имеет форму монеты $\{x:|y|<4,|z|<h / 2\}$, а оси круговых стержней с радиусом $h / 2$ и длиной 1 направлены в плоскости $\{x$ : $z=0\}$ вдоль нормалей к кругу в точках, образующих правильный $J$-угольник $(J \geqslant 3)$. Нетрудно убедиться в том, что требование $\left.4^{\circ}\right)$ соблюдено. Жесткое смещение $v(x)=\left(b_{1}-b_{6} y_{2}, b_{2}+b_{6} y_{1}, 0\right)^{\top}$ продолжается с пластины на стержни по правилу, описанному в примере 3.2. При этом $\left|v ; \Omega^{0}(h)\right|^{2} \geqslant c h, \mathscr{E}_{\mu}(v ; \Xi(h)) \leqslant$ $C \mu h^{2}$, а последнее соотношение верно потому, что благодаря ограничению $4^{\circ}$ ) не могут обращаться в нуль все проекции вектора $v$ на оси стержней. Таким образом, для "не жестких" ручек рулевого колеса (при $\left.\mu<h^{-1}(1+|\log h|)^{-2}\right)$ множитель $\beta^{\prime}(h, \mu)$ указан в теореме 5.4 правильно. Те же соображения, что и в примере $3.2,3)$, подтверждают необходимость условия $4^{\circ}$ ). 
5.6. Обсуждение. Теоремы 5.3 и 5.4 демонстрируют, что утверждение теоремы 5.2 и, в частности, распределение весовых множителей в неравенстве Корна (5.11), которое выполняется и при более слабом ограничении $3^{\circ}$ ), не являются оптимальными. По-видимому, дело обстоит так, что при фиксации пластины трехмерное условие $1^{\circ}$ ) из п. 2.2 и п. 3.2 перестает играть главенствующую роль и заменяется двумерными аналогами $\left.4^{\circ}\right)$ и $3^{\circ}$ ). Отметим, что последнее требование можно переформулировать так: $\mathbb{R}^{3}=\mathfrak{L}\left\{d^{b}\left(P^{j}\right)^{\top} \mid j=1, \ldots, J\right\}$. Здесь $d^{b}=\left(1, \alpha y_{2}, \alpha y_{1}\right)^{\top}$ - столбец, из которого вместе с $(2 \times 3)$-матрицей $(5.12)$ можно добавлением нулей соорудить $(3 \times 6)$-матрицу $d(y, 0)$, определенную в $(2.8)$ и фигурирующую в упомянутом условии $\left.1^{\circ}\right)$. Из теорем 5.3 и 5.4 вытекает следующее утверждение.

СлЕДСТВИЕ 5.1 [40]. Если $J \geqslant 6$ и условия $\left.3^{\circ}\right), 4^{\circ}$ ) выполнены одновременно, то для поля $u \in \stackrel{\circ}{H^{1}}(\Xi(h) ; \Gamma(h))^{3}$ справедливо неравенство Корна (5.11) с множителями

$$
\beta^{\prime}(h, \mu)=\min \left\{(1+|\log h|)^{-2}, h \mu\right\}, \quad \beta_{z}(h, \mu)=\min \left\{(1+|\log h|)^{-2}, h^{-1} \mu\right\} .
$$

Таким образом, упомянутый в примере 5.2 таракан, забившись в угол, может расположить свои шесть ног так, чтобы наилучшим образом сопротивляться произвольно направленным воздействиям.

В сравнении с предыдущими параграфами увеличилось количество пороговых значений жесткостного параметра $\mu$ : в теоремах $5.1,5.4$ фигурирует $\mu=h^{-1}$, в теоремах 5.1, 5.4 и $5.3-\mu=h^{-3}$, а в теоремах 5.4 и 5.3 - также $\mu=h$. Дело в том, что и пластина и стержни характеризуются жесткостями на изгиб и растяжение, имеющими существенно различающиеся порядки относительно параметра $h$. Так, величина $h^{3} \mu$ (величина $h^{-1} \mu$ ) является отношением жесткости стержня на изгиб (на растяжение) к жесткости пластины на растяжение (на изгиб). Величина $h \mu$ равна отношениям одноименных жесткостей стержней и пластины.

Обсудим один из важных трюков, способствующих достижению асимптотической точности в неравенстве Корна (см. прием 6) в п. 1.3). Алгебраические связи для компонент столбца $b^{0}$ из представления (5.3) поля смещений на столешне всегда следует получать, интегрируя по цилиндрам из более жесткого материала. Поэтому в случае $\mu<1$ оценки составляющей $u^{\perp}$ на $\mathscr{C}_{h}^{j}$ следует перевести на цилиндр $\mathscr{C}_{h}^{j 0} \subset \Omega^{0}(h)$, полученный параллельном переносом цилиндра $\mathscr{C}_{h}^{j}$. Это делается при помощи следующего утверждения.

ПреДЛОЖЕНИЕ 5.1 [39]. Пусть $\mathscr{C}_{h}^{j} \subset \Omega^{0}(h) \cap G_{h}^{j} u \mathscr{C}_{h}^{j 0} \subset \Omega^{0}(h)-$ иилиндрьь с одинаковыми размерами и параллельными осями, удаленными одна от другой на расстояние $O(h)$. Предположим, что выпуклая оболочка $\mathfrak{H}$ этих цилиндров содержится в $\Omega_{h}^{0}$. Тогда для поля $u \in H^{1}(\mathfrak{H})^{3}$ имеет место неравенство

$$
\begin{aligned}
& \min \{1, \mu\}\left\{\left\|u_{3}^{j} ; L_{2}\left(\mathscr{C}_{h}^{j 0}\right)\right\|^{2}+\sum_{i=1}^{2}\left(h^{2}\left\|u_{i}^{j} ; L_{2}\left(\mathscr{C}_{h}^{j 0}\right)\right\|^{2}+\left\|u_{i}^{j}-\overline{u_{i}^{j}} ; L_{2}\left(\mathscr{C}_{h}^{j 0}\right)\right\|^{2}\right)\right\} \\
& \quad \leqslant c\left\{\mathscr{E}_{\mu}(u ; \mathfrak{H})+\left\|u_{3}^{j} ; L_{2}\left(\mathscr{C}_{h}^{j}\right)\right\|^{2}+\sum_{i=1}^{2}\left(h^{2}\left\|u_{i}^{j} ; L_{2}\left(\mathscr{C}_{h}^{j}\right)\right\|^{2}+\left\|u_{i}^{j}-\overline{u_{i}^{j}} ; L_{2}\left(\mathscr{C}_{h}^{j}\right)\right\|^{2}\right)\right\},
\end{aligned}
$$


где $u_{i}^{j}$ и $u_{3}^{j}$ - проекции вектора и на оси $y_{i}^{j}$ и $z^{j}$ системы координат, привязанной $к$ стержню $G_{h}^{j}$, а остальные обозначения такие же, как в следствии 2.2.

Автору известна лишь одна публикация [59], посвященная асимптотическому анализу сочленения пластина/стержень, изображенного на рис. 11. При этом пластина и стержень предполагаются жестко защемленными по боковой поверхности и выступающему торцу соответственно, а значит, вопрос о неравенстве Корна решается применением теорем 2.6 и 2.9.

\section{7. Стержни, пронизывающие пластину в продольном направле-} нии. Пусть области $\omega^{0}, \omega^{1}, \ldots, \omega^{J}$ и $\Omega_{h}^{0}, Q_{h}^{1}, \ldots, Q_{h}^{J}$ такие же, как и в п. 5.1 , но предположим дополнительно, что оси стержней $\Lambda^{1}, \ldots, \Lambda^{J}$ лежат в плоскости пластины $\{x: z=0\}$, пересекают область $\omega^{0}$ по интервалам $\left(-l_{-}^{j 0}, l_{+}^{j 0}\right) \ni z^{j}$ и при некотором $\lambda>0$ справедливы включения

$$
\left\{x: \eta^{j} \in \omega^{j}, z^{j} \in\left(\lambda-l_{-}^{j}, l_{+}^{j}-\lambda\right)\right\} \subset \Omega_{h}^{0} .
$$

Стержень $G_{h}^{j}$ отсечен от цилиндра $Q_{h}^{j}$, имеет ось $\Upsilon^{j}=\left(-l_{-}^{j}, l_{+}^{j}\right) \ni z^{j}$, где $l_{-}^{j}<l_{-}^{j 0}$ и $l_{+}^{j}>l_{+}^{j 0}$, и его торец $\Gamma_{h}^{j}=\left\{x \in Q_{h}^{j}: z^{j}=l_{+}^{j}\right\}$, выступающий из пластины, жестко защемлен. Теперь множество $\Omega^{0}(h)=\Omega_{h}^{0} \backslash\left(\overline{G_{h}^{1}} \cup \cdots \cup \overline{G_{h}^{J}}\right)-$ пластина $\Omega_{h}^{0}$ с тонкими продольными каналами $g_{h}^{j}=\Omega_{h}^{0} \cap G_{h}^{j}$ (см. рис. 18).

В гнезда $g_{h}^{j}$ можно поместить круговые цилиндры $\mathscr{C}_{h}^{j}$ с радиусом $R h$ и высотой $2 H$. Это наблюдение дает возможность по прежней схеме установить следующее утверждение.

ТЕОРема 5.5. Для поля $u \in \stackrel{\circ}{H^{1}}\left(\Xi(h) ; \Gamma_{h}^{1} \cup \cdots \cup \Gamma_{h}^{J}\right)^{3}$ справедливо неравенство Корна (5.11), где $\mathscr{E}_{\mu}(u ; \Xi(h))$ - квадратичная форма (5.2), $\mid u^{j} ; G_{h}^{j} \mathbf{|}_{j}$ норма (2.52) при $\rho_{h}\left(z^{j}\right)=h+\left|z^{j}-l_{+}^{j}\right|$, постоянная с не зависит от параметров $h \in\left(0, h_{0}\right], \mu>0$ и вектор-функции $u$, а множители $\beta^{\prime}(h, \mu)$ u $\beta_{z}(h, \mu)$ в норме (2.48) определяются следующим образом:

1) если все прямые $\Lambda^{1}, \ldots, \Lambda^{J}$ параллельны одна другой или пересекаются в одной точке, то $\beta^{\prime}(h, \mu)=\min \left\{1, h^{3} \mu\right\}$ u $\beta_{z}(h, \mu)=\min \{1, h \mu\}$;

2) если среди $\Lambda^{1}, \ldots, \Lambda^{J}$ найдутся две пересекающиеся прямые и третъя, не проходящая через точку их пересечения, $\operatorname{mo} \beta^{\prime}(h, \mu)=\beta_{z}(h, \mu)=\min \{1, h \mu\}$.

Отметим, что опять геометрическое условие в теореме $5.5,2)$, приводящее к наилучшей норме $\boldsymbol{\|} \cdot ; \Omega^{0}(h) \boldsymbol{|}_{\beta}$ в правой части неравенства Корна, равносильно требованию $4^{\circ}$ ). Наоборот, в теореме $\left.5.5,1\right)$ коразмерность линейной оболочки из упомянутого требования положительна (равна 1 при $J \geqslant 2$ или 2 при $J=1)$.

ПримеР 5.5. Железобетонная плита. Введем множества, изображенные на рис. 18, формулами $\Omega_{h}^{0}=\left\{x:\left|y_{i}\right|<2,|z|<2 h\right\}$ и

$$
\begin{aligned}
G_{h}^{i} & =\left\{x:\left|y_{2}-(-1)^{i}\right|^{2}+z^{2}<h^{2},\left|y_{1}\right|<5 / 2\right\}, \quad i=1,2, \\
G_{h}^{3} & =\left\{x:\left|y_{1}\right|^{2}+z^{2}<h^{2},\left|y_{2}\right|<5 / 2\right\} .
\end{aligned}
$$

Если сочленение $\Xi$ образовано пластиной $\Omega_{h}^{0}$ и стержнями $G_{h}^{1}$ и $G_{h}^{2}$, то положим $u(x)=e_{2}$ при $x \in \Omega_{h}^{0}$ и $u(x)=e_{2} \chi\left(2 y_{1}\right)-\left(y_{2}-(-1)^{i}\right) e_{1} \partial_{1} \chi\left(2 y_{1}\right)$ при $x \in G_{h}^{i}$, 


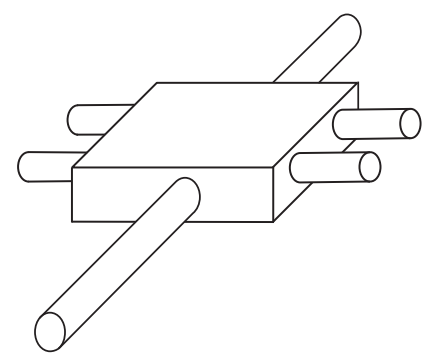

Рис. 18. Железобетонная плита

$i=1,2$. При этом

$$
\| u ; \Omega^{0}(h) \boldsymbol{|}^{2} \geqslant c h, \quad \mathscr{E}_{\mu}(u ; \Xi(h)) \leqslant C \mu h^{4},
$$

а значит, при "не очень жесткой" арматуре $\left(h^{3} \mu \ll 1\right.$ и $\left.h \mu>1\right)$ неравенство (5.11) с множителем $\beta^{\prime}(h, \mu)=1$ невозможно.

В случае сочленения $\Xi(h)=\Omega_{h}^{0} \cup G_{h}^{2} \cup G_{h}^{3}$ (убран один из параллельных стержней на рис. 18) поле, приводящее к соотношениям (5.13), имеет вид

$$
\begin{array}{lll}
u(x)=e_{1}\left(1-y_{2}\right)+e_{1} y_{2}, & x \in \Omega_{h}^{0}, \\
u(x)=e_{2} y_{1} \chi\left(2 y_{1}\right)-\left(1-y_{2}\right) e_{1} \partial_{1} y_{1} \chi\left(2 y_{1}\right), & & x \in G_{h}^{2}, \\
u(x)=e_{1}\left(1-y_{2}\right) \chi\left(2 y_{2}\right)+y_{1} e_{1} \partial_{2}\left(1-y_{2}\right) \chi\left(2 y_{2}\right), & & x \in G_{h}^{3} .
\end{array}
$$

Наконец, при трех пронизывающих стержнях не удается продолжить какоелибо жесткое смещение с пластины на стержни $G_{h}^{q}, q=1,2,3$, при сохранении соотношения (5.13) - аргументы такие же, как и в примере $3.2,3$ ).

5.8. Стержень, присоединенный к закрепленной пластине. Пусть пластина $\Omega_{h}^{0}$ защемлена вдоль множества $\Gamma_{h}^{0}$, заданного формулой $(4.5)$, где $\gamma_{h}^{0} \subset \partial \omega^{0}-$ непустая открытая дуга. Рассмотрим один из стержней $G_{h}^{1}$, определенных в п. 5.1, но его торец $\Gamma_{h}^{10}$ вне пластины будем считать свободным, т. е. для него не поставим условие $\left(2.18_{1}\right)$.

ТЕОРема 5.6 [40]. Для поля $u \in \stackrel{\circ}{H^{1}}\left(u ; \Omega_{h}^{0} \cup G_{h}^{1}, \Gamma_{h}^{10}\right)^{3}$ справедливо неравенство Корна

$$
\mathbf{\|}\left|u^{0} ; \Omega^{0}(h)\left\|_{0}^{2}+\sigma(h, \mu) \mid u^{1} ; G_{h}^{1}\right\|_{1}^{2} \leqslant c \mathscr{E}_{\mu}\left(u ; \Omega_{h}^{0} \cup G_{h}^{1}\right),\right.
$$

где $\mathbf{I} \cdot ; \Omega_{h}^{0} \mathbf{I}_{0} u \mathbf{|} \cdot ; G_{h}^{1} \mathbf{I}_{1}-$ нормы (2.42) u (2.52) при $\rho_{h}(y)=h+\operatorname{dist}\left(y, \gamma^{0}\right) u$ $\rho_{h}\left(z^{1}\right)=1$ соответственно, а множитель в левой части имеет вид

$$
\sigma(h, \mu)=\min \left\{h\left(\sin \theta_{1}+h^{2}\right)^{-1}(1+|\log h|)^{-2}, \mu\right\},
$$

причем $\theta_{1}-$ угол между осъю стержня и плоскостью пластины.

Формула (5.14) различает сочленения, изображенные на рис. 11 и 12 , у которых $\theta_{1}=\pi / 2$ и $\theta_{1}=0$ соответственно. Так, например, для однородного $(\mu=1)$ сочленения множитель $\sigma(h, 1)$ равен $h(1+|\log h|)^{-2}$ в первом случае и 1 во втором. Объяснением служит то, что при расположении стержня в плоскости пластины продольные направления в тонких телах согласованы, а в остальных случаях - рассогласованы. 


\section{§ 6. Неравенства Корна для сочленения тонких пластин}

6.1. Геометрия сочленений. Пусть $\Pi^{0}$ и $\Pi^{1}, \ldots, \Pi^{J}-$ плоскости в пространстве $\mathbb{R}^{3}$, с которыми связаны декартовы системы координат $x=\left(y^{0}, z^{0}\right)$ и $\left(y^{1}, z^{1}\right), \ldots,\left(y^{J}, z^{J}\right)$, причем ось $z^{p}$ направлена перпендикулярно плоскости $\Pi^{p}, p=0,1, \ldots, J$. Пусть $\omega^{p} \subset \Pi^{p}$ - область с кусочно гладкой границей $\partial \omega^{p}$ и компактным замыканием $\overline{\omega^{p}}$. Введем формулой (4.1) тонкие пластины $\Omega_{h}^{p}$. Как обычно, не различаем в обозначениях двумерные множества, снабженные индексом $p$, и их погружения в $\mathbb{R}^{3}$ на плоскость $\Pi^{p}$. Функции $H_{ \pm}^{p}$, определяющие переменные толщины пластин без учета малого параметра $h \in\left(0, h_{0}\right]$, для простоты также предполагаем кусочно гладкими. В данном параграфе индексы $p$ и $j$ принимают значения $1, \ldots, J$ и $0,1, \ldots, J$ соответственно; для других целей эти литеры не используются. Пластину $\Omega_{h}^{0}$ называем основной и на ее границе условий Дирихле $\left(2.18_{1}\right)$ не ставим.

Предположим, что пластины $\Omega_{h}^{1}, \ldots, \Omega_{h}^{J}$, во-первых, образуют непустые пересечения $g_{h}^{j}=\Omega_{h}^{0} \cap \Omega_{h}^{j}$ с основной пластиной, во-вторых, они жестко защемлены вдоль частей (4.5) боковых поверхностей вне множества $\overline{\Omega_{h}^{0}}$ и, в-третьих, отношение жесткостей материалов, из которых изготовлены упругие тела $\Omega_{h}^{1}, \ldots, \Omega_{h}^{J}$ и $\Omega_{h}^{0}$ соответственно, равно $\mu$. Иными словами, рассматривается подобный (2.56) и (4.3) эрзац упругой энергии

$$
\mathscr{E}_{\mu}(u ; \Xi(h))=\left\|D\left(\nabla_{x}\right) u^{0} ; L_{2}\left(\Omega^{0}(h)\right)\right\|^{2}+\mu \sum_{j=1}^{J}\left\|D\left(\nabla_{x}\right) u^{j} ; L_{2}\left(\Omega^{j}(h)\right)\right\|^{2},
$$

где $u \in H^{1}(G(h))^{3}, u^{p}=\left.u\right|_{\Omega_{h}^{p}}$, а пластины $\Omega^{p}(h)$ в сочленении $\Xi(h)=\Omega^{0}(h) \cup$ $\Omega^{1}(h) \cup \cdots \cup \Omega^{J}(h)$ определены разными формулами в зависимости от способа крепления. Именно, при вставке пластин $\Omega_{h}^{j}$ в пазы $g_{h}^{j}$ положим

$$
\Omega^{0}(h)=\Omega_{h}^{0} \backslash\left(\overline{\Omega_{h}^{1}} \cup \cdots \cup \overline{\Omega_{h}^{J}}\right), \quad \Omega^{j}(h)=\Omega_{h}^{j} .
$$

Если же пластины $\Omega^{j}(h)$ приклеены к поверхности пластины $\Omega_{h}^{0}$, то

$$
\Omega^{0}(h)=\Omega_{h}^{0}, \quad \Omega^{j}(h)=\Omega_{h}^{j} \backslash \overline{\Omega_{h}^{0}} .
$$

Примем следующие условия:

$\left.1^{\circ}\right)$ если $\mu<1$, то справедливо неравенство

$$
\left|u^{0} ; \Omega^{0}(h)\right|^{2} \leqslant c\left(\mathscr{E}_{\mu}\left(u^{0} ; \Omega^{0}(h)\right)+\left|F^{* h}\left(u^{0}\right)\right|^{2}\right),
$$

в котором замыкание области $\omega^{*}$ в определении (2.40) функционала $F^{* h}$ содержится внутри множества $\omega \backslash\left(\Pi^{1} \cap \cdots \cap \Pi^{J}\right)$;

$2^{\circ}$ ) если $\mu>1$, то справедливо неравенство

$$
\mathbf{\|} u^{j} ; \Omega^{j}(h) \mathbf{|}^{2} \leqslant c \mathscr{E}_{\mu}\left(u^{j} ; \Omega^{0}(h)\right), \quad u \in \stackrel{\circ}{H^{1}}\left(\Omega^{0}(h) ; \Gamma_{h}^{j}\right)^{3} .
$$

Эти допущения позволяют унифицировать формулируемые далее утверждения. 
ЗАмечАниЕ 6.1. 1) О неравенствах Корна для пластин с возмущенными кромками речь шла в п. 2.4, и нетрудно изменить приведенное там условие $3^{\circ}$ ) для обеспечения соотношения (6.5).

2) Для того чтобы найти условия, гарантирующие справедливость неравенства Корна (6.4), следует разбить область $\omega$ плоскостями $\Pi^{1}, \ldots, \Pi^{J}$ на подобласти $\omega^{0 n}, n=1, \ldots, N$, и выбрать срединное сечение $\omega^{*} \subset \omega^{n}$ области интегрирования в функционале $(2.40)$ при каком-либо $n=n_{*}$. Затем можно получить неравенство вида (6.5) на пластине $\Omega^{0 n_{*}}=\left\{(y, z): y \in \omega^{0 n_{*}}\right\}$ и передать его с $\Omega^{0 n_{*}}$ на остальные пластины $\Omega^{0 n}, n \neq n_{*}$, сначала на соседние, затем на их окружение и т.д. При этом требуется условие $2^{\circ}$ ) для каждой из пластин $\Omega^{0 n}$, а также новое предположение о том, что общая часть боковых поверхностей соседних пластин $\Omega^{0 n}$ и $\Omega^{0 m}$ содержит непустое множество $\Gamma^{n m *}=\left\{(y, z): y \in \gamma^{n m *},-h H_{-}^{n m *}<z<h H_{+}^{n m *}\right\}$, где $\gamma^{n m *}-$ дуга положительной длины на $\partial \omega^{0 n} \cap \partial \omega^{0 m}$. Подобное (2.37) неравенство с областью интегрирования $\Gamma^{n m *}$ в функционале $\left(2.35_{2}\right)$ дает возможность осуществить указанные передачи.

Формулировки упомянутых в замечании 6.1 геометрических требований, допускающие липшицевы области, достаточно громоздки. При кусочно гладких контурах $\partial \omega^{p}$ и функциях $H_{ \pm}^{p}$ неравенства Корна (6.4) и (6.5) обычно выполнены, за исключением патологических ситуаций, отмеченных в следующем примере.

ПримеР 6.1. Пусть сочленение образовано двумя пластинами $\Omega_{h}^{0}$ и $\Omega_{h}^{1}$, причем $H_{+}^{0}=1, H_{-}^{0}=0, H_{ \pm}^{1}=1 / 2$,

$$
\begin{aligned}
& \omega^{0}=\left\{(y, z):\left|y_{1}\right|<2,\left|y_{2}\right|<2, z=0\right\}, \\
& \omega^{1}=\left\{(y, z):\left|y_{1}\right|<2, y_{2}=0,-F\left(y_{1}\right)<z<0\right\},
\end{aligned}
$$

$F$ - четная функция, монотонно убывающая от 1 до -1 на отрезке $[0,2] \ni y_{1}$ и в окрестности точки $y_{1}=1$ имеющая вид $F\left(y_{1}\right)=\left(1-y_{1}\right)^{1+\delta}, \delta>0$. Система координат $\left(y^{1}, z^{1}\right)$, привязанная к пластине $\Omega_{h}^{1}$, задана равенствами $y_{1}^{1}=y_{1}$, $y_{2}^{1}=z$ и $z^{1}=y_{2}$. Пластина $\Omega_{h}^{1}$ рассекается плоскостью $\Pi^{0}$ на две части $\Omega_{h}^{1 \pm}=\left\{(y, z) \in \Omega_{h}^{1}: \pm z>0\right\}$. Области $\Omega^{0}(h)=\Omega_{h}^{0} \backslash \overline{\Omega_{h}^{1+}}$ и $\Omega^{1}(h)=\Omega_{h}^{1-}$, определенные по формулам (6.2) и (6.3), имеют нулевые заострения на границе, т. е. на них неравенство Корна не выполняется (см. пример 2.1). Отметим также простейший случай нарушения неравенства (6.4): область $\omega^{1}$ в формуле $\left(6.6_{2}\right)$ - прямоугольник $\left\{(y, z):\left|y_{1}\right|<3, y_{2}=0,|z|<3\right\}$ и, значит, пластина $\Omega^{0}(h)$ - несвязное множество.

В следующем пункте на расположение закрепленных пластин $\Omega_{h}^{1}, \ldots, \Omega_{h}^{J}$ по отношению к основной $\Omega_{h}^{0}$ не накладываем никаких ограничений. В то же время, составная пластина была изучена в п. 2.7 и, если пластина $\Omega_{h}^{j}$ служит продолжением пластины $\Omega_{h}^{0}$, из предложения 2.4 вытекает неравенство Корна

$$
\min \{1, \mu\}\left|u ; \widetilde{\Omega}_{h}^{0}\right|^{2}+\left|u ; \widetilde{\Omega}_{h}^{j}\right|^{2} \leqslant c \mathscr{E}_{\mu}(u ; \Xi(h)),
$$

которое, вообще говоря, нельзя улучшить за счет других пластин при произвольном расположении последних (исключение описано в примере $6.3,2$ )). 
Поэтому в данном параграфе считаем, что при $j=1, \ldots, J$ плоскости $\Pi^{j}$ пересекают $\Pi^{0}$ под ненулевыми углами $\theta_{j}$. Иными словами, множества $g_{h}^{j}=\Omega_{h}^{0} \cap \Omega_{h}^{j}$ оказываются тонкими продолговатыми телами. В каждом из них можно выбрать круговой цилиндр $\mathscr{C}_{h}^{j}$ с радиусом основания $R h$ и высотой $2 H$. Системы координат $\left(y^{j}, z^{j}\right)$ зафиксируем так, чтобы эти цилиндры имели вид

$$
\mathscr{C}_{h}^{j}=\left\{\left(y^{j}, z^{j}\right):\left|y_{1}^{j}\right|<H,\left|y_{2}^{j}\right|^{2}+\left|z^{j}\right|^{2}<R^{2} h^{2}\right\}
$$

(ср. п. 4.2). Не исключается случай, когда для двух или более пластин линии пересечения $\Lambda^{j}=\Pi^{0} \cap \Pi^{j}$ совпадают (см. далее рис. 21). Подчеркнем, что в случае липшицевых границ существование цилиндров (6.8) внутри пересечений $g_{h}^{j}=\Omega_{h}^{0} \cap \Omega_{h}^{j}$ нужно предполагать.

В п. 6.3 будут востребованы три геометрических условия.

$3^{\circ}$ ) Среди плоскостей $\Pi^{1}, \ldots, \Pi^{J}$ найдутся три различные плоскости, две из которых пересекаются, а третъя не содержит эту линию пересечения целиком.

$4^{\circ}$ ) Существуют две плоскости $\Pi^{j} \neq \Pi^{m}$, линия пересечения которых лежит на плоскости $\Pi^{0}$.

$5^{\circ}$ ) Существуют две плоскости $\Pi^{j} \neq \Pi^{m}$, перпендикулярные плоскости $\Pi^{0}$.

Геометрические условия допускают алгебраическую формулировку. Определим линеалы

$$
\begin{aligned}
\mathfrak{L}_{3} & :=\mathfrak{L}\left\{d^{\sharp}(y, 0)^{\top} a^{\sharp} \mid(y, 0) \in \Upsilon^{j}:=\omega^{0} \cap \omega^{j},\right. \\
& \left.a^{\sharp} \in \mathbb{R}^{2} \text { лежит на } \Lambda^{j}, j=1, \ldots, J\right\} \subset \mathbb{R}^{3}, \\
\mathfrak{L}_{6}: & =\mathfrak{L}\left\{d(y, 0)^{\top} a \mid(y, 0) \in \Upsilon^{j}, a \in \mathbb{R}^{3} \text { лежит на } \Pi^{j}, j=1, \ldots, J\right\} \subset \mathbb{R}^{6},
\end{aligned}
$$

в которых фигурируют матрицы (5.12) и (2.8). Введем еще укороченную третью строку $(3 \times 6)$-матрицы $d(y, 0)$, а именно $d^{\sharp \sharp}(y)=\left(1,2^{-1 / 2} y_{2},-2^{-1 / 2} y_{1}\right)$. Отметим, что блоки $d^{\sharp \sharp}(y)$ и $d^{\sharp}(y, 0)$ вобрали в себя все нетривиальные элементы упомянутой $(3 \times 6)$-матрицы.

ЛЕмма 6.1 [60], [61]. 1) При $J=1$ оболочка $\mathfrak{L}_{3}$ (оболочка $\mathfrak{L}_{6}$ ) одномерна (двумерна).

2) Если $J>1$ и плоскости $\Pi^{2}, \ldots, \Pi^{J}$ параллельны $\Pi^{1}$ или пересекаются c $\Pi^{1}$ по общей прямой, не лежащей на плоскости $\Pi^{0}$, но хотя бы одна из них не coвnaдаem с $\Pi^{1}$, mo $\operatorname{dim} \mathfrak{L}_{3}=2 u \operatorname{dim} \mathfrak{L}_{6}=5$.

3) Если выполнено условие $\left.3^{\circ}\right)$, то $\mathfrak{L}_{3}=\mathbb{R}^{3}$ и $\mathfrak{L}_{6}=\mathbb{R}^{6}$.

4) Если в определении $\left(6.9_{1}\right)$ в качестве столбца $a^{\sharp} \in \mathbb{R}^{2}$ выступает любой вектор на плоскости $\Pi^{0}$, то условие $4^{\circ}$ ) гарантирует, что $\mathfrak{L}_{3}=\mathbb{R}^{3}$.

5) Условие $\left.5^{\circ}\right)$ обеспечивает равенство $\mathfrak{L}\left\{d^{\sharp \sharp}(y)^{\top} b_{3} \mid(y, 0) \in \Upsilon^{j}=\omega^{0} \cap \omega^{j}\right.$, $\left.b_{3} \in \mathbb{R}, j=1, \ldots, J\right\}=\mathbb{R}^{3}$.

\section{2. Неравенства Корна без ограничений на строение сочленения.}

Сначала рассмотрим случай вставки поддерживающих пластин в пазы.

Теорема 6.1 [61]. Пусть пластины в сочленении $\Xi(h)$ определены формулами (6.3) при выполнении условия $2^{\circ}$ ) или формулами (6.2). Тогда для поля 
$u \in \stackrel{\circ}{H}^{1}\left(\Xi(h) ; \Gamma_{h}^{1} \cup \cdots \cup \Gamma_{h}^{J}\right)^{3}$ справедливо неравенство Корна

$$
\mathbf{I}\left|u^{0} ; \Omega^{0}(h) \mathbf{|}_{\beta}^{2}+\mu \sum_{j=1}^{J}\right| u^{j} ; \Omega^{j}(h) \mathbf{|}_{j}^{2} \leqslant c \mathscr{E}_{\mu}(u ; \Xi(h)),
$$

где $\mid \cdot ; \Omega_{h}^{j} \mathbf{I}_{j}$ - норма (2.42) при $\rho_{h}\left(y^{j}\right)=h+\operatorname{dist}\left(y^{j}, \gamma^{j}\right)$, а норма (2.48), первая в левой части (6.10), содержит множители

$$
\beta^{\prime}(h, \mu)=\min \left\{1, h^{2} \mu\right\}, \quad \beta_{z}(h, \mu)=\min \{1, \mu\} .
$$

При нарушении требования $2^{\circ}$ ) в теореме 6.1 приходится положить $\beta^{\prime}(h, \mu)=$ $h^{2} \min \{1, \mu\}$. Этот весовой множитель отличается от приведенного в $(6.11)$ только для "жестких" пластин $\Omega^{j}(h)$, т. е. при $\mu>1$.

\section{3. Неравенство Корна при специальном строении сочленения.} Если цилиндр (6.8) принадлежит одновременно трем разным пластинам $\Omega_{h}^{j}, \Omega_{h}^{m}$ и $\Omega_{h}^{0}\left(\right.$ ср. условие $\left.4^{\circ}\right)$ и рис. 21$)$, то по обычной схеме весовые множители в неравенстве Корна могут быть увеличены.

ТеОРема 6.2 [61]. Пусть пластины в сочленении $\Xi(h)$ определены формулами (6.2) или (6.3) при выполнении условий $\left.1^{\circ}\right)$ и $2^{\circ}$ ) соответственно. Тогда справедливо утверждение теоремы 6.1, причем весовые множители в весовой анизотропной норме (2.48), фигурирующей в неравенстве Корна (6.10), определены следующим образом:

1) при соблюдении условия $3^{\circ}$ )

$$
\beta^{\prime}(h, \mu)=\min \{1, \mu\}, \quad \beta_{z}(h, \mu)=\min \left\{1, h^{-2} \mu\right\} ;
$$

2) если справедливо условие $\left.4^{\circ}\right)$, то множители $\beta^{\prime}(h, \mu)$ и $\beta_{z}(h, \mu)$ берутся из формул (6.12) и (6.11) соответственно;

3) если справедливо условие $\left.5^{\circ}\right)$, то множители $\beta^{\prime}(h, \mu)$ и $\beta_{z}(h, \mu)$ берутся из формул (6.11) и (6.12) соответственно.

6.4. Обсуждение. Число $h^{2} \mu$ (число $h^{-2} \mu$ ) в теореме 6.1 (в теореме 6.2), есть отношение жесткости на изгиб $h^{3} \mu$ (на растяжение $h \mu$ ) закрепленных пластин $\Omega_{h}^{1}, \ldots, \Omega_{h}^{J}$ к жесткости на растяжение $h$ (на изгиб $h^{3}$ ) поддерживаемой пластины $\Omega_{h}^{0}$.

Если пластины $\Omega_{h}^{1}, \ldots, \Omega_{h}^{J}$ достаточно жесткие $(\mu>1)$ или очень жесткие $\left(\mu>h^{-2}\right)$, то в любом случае $\beta^{\prime}(h, \mu)=1$ или $\beta_{z}(h, \mu)=1$. В таких условиях вопрос о точности неравенства Корна (6.10) решается при помощи поля смещений (2.10), причем носители функций $w_{i}$ должны не пересекаться с множествами $g_{h}^{1}, \ldots, g_{h}^{J}$. Проверим правильность подбора весовых множителей $\beta^{\prime}(h, \mu)$ и $\beta_{z}(h, \mu)$ в норме ||$\cdot ; \Omega^{0}(h) \mid \mathbf{I}_{\beta}$ для мягких или не очень жестких поддерживающих пластин.

ПримеР 6.2. Стонхендж. Крупнейшая мегалитическая постройка эпохи неолита (см., например, [62]) состоит из различных комбинаций вертикальных и горизонтальных каменных плит, в частности, содержит трилиты, изображенные на рис. 19-21. 


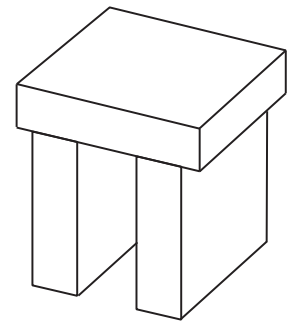

Рис. 19

Первый трилит

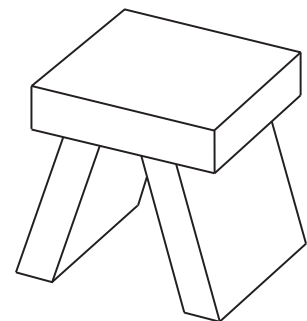

Рис. 20

Второй трилит

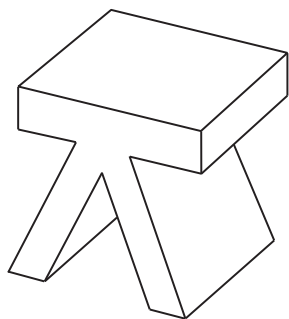

Рис. 21

Третий трилит

1) Скошенные опоры. Пусть, как на рис. $20, \Omega_{h}^{0}=\left\{x=(y, z):\left|y_{1}\right|<1\right.$, $\left.\left|y_{2}\right|<1,|z|<h / 2\right\}$, а продольные сечения $\omega^{1}$ и $\omega^{2}$ пластин $\Omega_{h}^{1}$ и $\Omega_{h}^{2}$, имеющих толщину $h$, вырезаны параллелепипедом $\left\{x:\left|y_{1}\right|<1,\left|y_{2}\right|<1,-3 / 2<z<0\right\}$ из плоскостей $\Pi^{1}$ и $\Pi^{2}$, проходящих через прямую $\Lambda(Z)=\left\{x: y_{2}=0, z=Z\right\}$ и обладающих нормалями $(0,1+Z,-1)^{\top}$ и $(0,1+Z, 1)^{\top}$ соответственно. Ни одно из требований $\left.3^{\circ}\right)-5^{\circ}$ ) не выполнено, а поле $u$, подтверждающее асимптотическую точность неравенства Корна из теоремы 6.1 , совпадает на перекрытии $\Omega_{h}^{0}$ с вектором поворота вокруг прямой $\Lambda(Z)$

$$
u^{0}(x)=e_{2}(z-Z)-e_{3} y_{2} .
$$

Поскольку вектор жесткого смещения (6.13) перпендикулярен обеим плоскостям $\Pi^{i}, i=1,2$, он может быть продолжен на опоры $\Omega_{h}^{i}$ так, что

$$
\mathscr{E}\left(u^{0} ; \Omega_{h}^{0}\right)=0, \quad \mathscr{E}\left(u^{i} ; \Omega_{h}^{i}\right)=O\left(h^{3}\right) .
$$

В самом деле, если ось $y_{1}^{i}$ системы координат $\left(y^{i}, z^{i}\right)$, соотнесенной с плоскостью $\Pi^{i}$, направлена вдоль $\Lambda(Z)$, а ось $y_{2}^{i}-$ от этой прямой к пластине $\Omega_{h}^{0}$, то нужное продолжение, построенное согласно формулам (2.43), выглядит следующим образом:

$$
u^{i}(x)=-e_{3}^{i} W_{Z}\left(y_{i}^{2}\right)+e_{2}^{i} z^{i} W_{Z}^{\prime}\left(y_{2}^{i}\right), \quad i=1,2 .
$$

Здесь $t \mapsto W_{Z}(t)$ - гладкая функция, равная единице при $t<T+1 / 2$ и нулю при $t>T+1, W_{Z}^{\prime}$ - ее производная, а $T=\left(Z^{2}+1\right)^{1 / 2}-$ расстояние между прямыми $\Lambda(Z)$ и $\Lambda^{i}$. В силу формулы (6.13) и неравенства $Z>0$ верны соотношения

$$
\mathbf{|} e_{1} u_{1}^{0}+e_{2} u_{2}^{0} ; \Omega_{h}^{0} \mathbf{|}^{2}=O(h), \quad \| e_{3} u_{3}^{0} ; \Omega_{h}^{0} \mathbf{|}^{2}=O\left(h^{3}\right),
$$

означающие, в частности, что множители (6.11) увеличить нельзя.

2) Опоры, сходящиеся в верхней точке. Пусть $Z=0$, а остальные определения в предыдущем примере сохранены. Сочленение, изображенное на рис. 21, удовлетворяет условию $4^{\circ}$ ), но не условиям $3^{\circ}$ ) и $5^{\circ}$ ). Соотношения $(6.14)$ сохраняются для поля (6.13), (6.15), но соотношения (6.16) заменяются такими: $\mathbf{|} e_{1} u_{1}^{0}+e_{2} u_{2}^{0} ; \Omega_{h}^{0} \mathbf{|}^{2}=O\left(h^{3}\right)$ и $\mid e_{3} u_{3}^{0} ; \Omega_{h}^{0} \mathbf{|}^{2}=O\left(h^{3}\right)$. Сравнивая упомянутые формулы, видим, что множители $\beta^{\prime}(h, \mu)=\min \{1, \mu\}$ и $\beta_{z}(h, \mu)=\min \{1, \mu\}$, указанные теоремой $6.2,2)$, оптимальны. 
3) Прямые опоры. Пусть теперь $Z=+\infty$, т. е. плоскости $\Pi^{1}$ и $\Pi^{2}$ перпендикулярны плоскости $\Pi^{0}$ и поэтому выполнено условие $5^{\circ}$ ), но не условия $3^{\circ}$ ) и $\left.4^{\circ}\right)$. Рассмотрим поле

$$
\begin{array}{llrl}
u^{0}(x) & =e_{2}, & & x \in \Omega_{h}^{0}, \\
u^{i}(x)=e_{2} W_{0}(z)-e_{3}\left(y_{2}+(-1)^{i}\right) W_{0}^{\prime}(z), & x \in \Omega_{h}^{i}, \quad i=1,2,
\end{array}
$$

для которого верны соотношения (6.14), а также первое из соотношений (6.16). Оно подтверждает правильность выбора весового множителя $\beta^{\prime}(h, \mu)=$ $\min \left\{1, h^{2} \mu\right\}$ в теореме $\left.6.2,3\right)$. Поскольку компонента $u_{3}^{0}$ тривиальная, множитель $\beta_{z}(h, \mu)=\min \left\{1, h^{-2} \mu\right\}$ обосновывается при помощи другого поля

$$
u^{0}(x)=e_{3}, \quad x \in \Omega_{h}^{0}, \quad u^{i}(x)=e_{3} W_{0}(z), \quad x \in \Omega_{h}^{i}, \quad i=1,2 ;
$$

при этом $\mathscr{E}\left(u^{0} ; \Omega_{h}^{0}\right)=0, \mathscr{E}\left(u^{i} ; \Omega_{h}^{i}\right)=O(h),\left|e_{1} u_{1}^{0}+e_{2} u_{2}^{0} ; \Omega_{h}^{0} \mathbf{|}^{2}=0,\right| e_{3} u_{3}^{0} ;\left.\Omega_{h}^{0}\right|^{2}=$ $O\left(h^{3}\right)$. Увеличение количества вертикальных опор не может усилить неравенство Корна из теоремы 6.2, 3): конкретные поля смещений (6.17) и (6.18) на сочленении, изображенном на рис. 22 , дают прежние границы для весовых множителей.

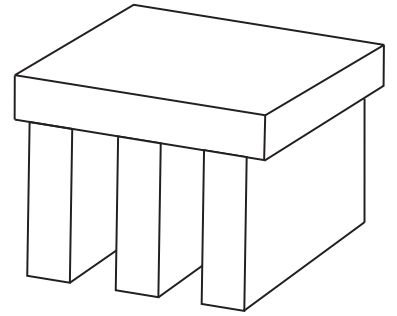

Рис. 22. Первый квадрилит

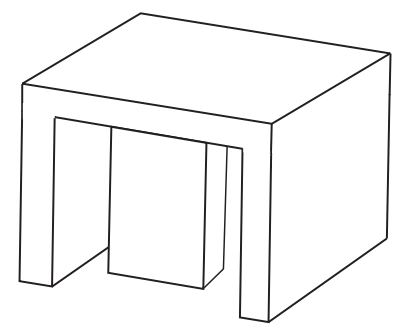

Рис. 23. Второй квадрилит

ПримеР 6.3. Сооружения из четырех плит. 1) Пусть сочленение составлено из четырех пластин так, как указано на рис. 23 или рис. 24, причем горизонтальная пластина - основная, а остальные три защемлены по нижним торцам. Нетрудно убедиться, что для сочленения на рис. 23 справедливо условие $3^{\circ}$ ), которое по теореме $6.2,1$ ) обеспечивает максимально возможные весовые множители (6.12). Подчеркнем, что благодаря средней опоре, развернутой вдоль поддерживаемой плиты, условие $3^{\circ}$ ) сохраняется и при боковых опорах, скошенных так, как указано на рис. 20. В последнем случае требования $4^{\circ}$ ) и $5^{\circ}$ ) нарушены. Они выполнены для сочленения на рис. 24 - теперь нарушено требование $3^{\circ}$ ), однако утверждения 2) и 3) теоремы 6.2 вместе предписывают те же весовые множители (6.12), что и утверждение 1).

2) В сочленении на рис. 25 горизонтальная плита составная и две поддерживающие плиты защемлены вдоль нижних и правого торцов. При этом соблюдено требование $5^{\circ}$ ), дозволяющее второй весовой множитель в (6.12). Первый допусти́м в силу неравенства (6.7), обслуживающего составную плиту. Отметим еще, что если упомянутая составная плита не защемлена по правому торцу, т. е. является основной, то не выполнены условие $1^{\circ}$ ) и неравенство Корна (6.4), а сочленение обслуживается теоремами $6.2,3)$ и 6.3 (см. следующий пункт). 


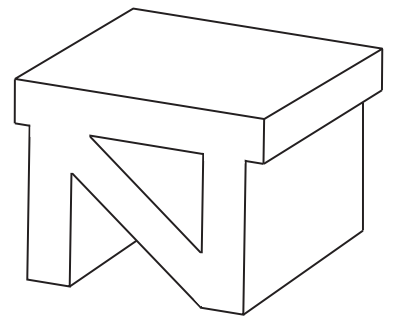

Рис. 24. Третий квадрилит

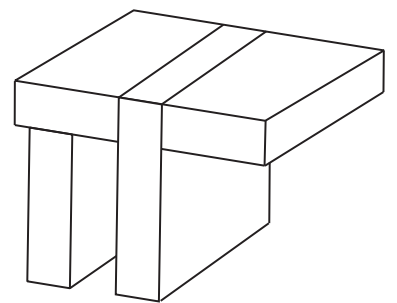

Рис. 25. Композитный трилит

\section{5. О передаче весового неравенства неравенства Корна с одной} пластины на другую. В этом пункте рассмотрим однородное $(\mu=1)$ сочленение. Теорема 2.6 позволяет оценить норму $\mid u^{1} ; \Omega_{h}^{1} \mathbf{l}_{0}$ на защемленной пластине $\Omega_{h}^{1}$, а теорема 6.1 или неравенство (6.7) - передать неравенство Корна на поддерживаемую пластину $\Omega_{h}^{0}$, оценив при этом норму $\mid u^{0} ; \Omega_{h}^{0} \mathbf{I}_{\beta}$ с множителями

$$
\beta^{\prime}(h, 1)=h^{2}, \quad \beta_{z}(h, 1)=1
$$

или $\beta^{\prime}(h, 1)=\beta_{z}(h, 1)=1$ соответственно. Очередное утверждение указывает правильные весовые множители в левой части неравенства

$$
\mathbf{\|} \mid u^{2} ; \Omega_{h}^{2} \|_{\sigma}^{2} \leqslant c\left(\mathscr{E}_{\mu}\left(u ; \Omega_{h}^{2}\right)+\mathbf{\|} u^{0} ; \Omega_{h}^{0} \| \boldsymbol{\|}_{\beta}^{2}\right)
$$

в том случае, если пластина $\Omega_{h}^{2}$ не защемлена, не пересекается с $\Omega_{h}^{1}$, но присоединена к $\Omega_{h}^{0}$.

ТЕОРема 6.3. При любом взаимном расположении пластин $\Omega_{h}^{0}$ и $\Omega_{h}^{2}$ справедливо неравенство Корна (6.20), в котором весовые множители $\sigma^{\prime}(h, 1)=$ $\beta^{\prime}(h, 1)$ и $\sigma_{z}(h, 1)=\beta_{z}(h, 1)$ имеют вид (6.19).

При помощи теоремы 6.3 можно анализировать сочленения, подобные ширмам или гармошкам. Например, если у однородного сочленения на рис. 22 защемлена лишь одна стойка, то оптимальными на перекрытии и двух других стойках оказываются весовые множители (6.19), которые не уменьшаются вне зависимости от углов соединения и количества прикрепленных пластин.

\section{§ 7. Неравенства Корна для сочленения тонких стержней}

7.1. Система стержней. Пусть область $\Xi(h)$, зависящая от малого параметра $h \in\left(0, h_{0}\right]$, является объединением тонких стержней $G_{h}^{1}, \ldots, G_{h}^{N}$ и малых узлов $Q_{h}^{1}, \ldots, Q_{h}^{M}$,

$$
\Xi(h)=\bigcup_{n=1}^{N} G_{h}^{n} \cup \bigcup_{m=1}^{M} Q_{h}^{m} .
$$

Для более точного описания области (7.1) произведем дополнительные построения. Некоторые пары точек из набора $\mathfrak{P}=\left\{P^{1}, \ldots, P^{N}\right\}$ соединим прямолинейными замкнутыми отрезками $\Sigma^{1}, \ldots, \Sigma^{N}$. Объединение $\bigcup \Sigma^{n}$ называем скелетом $\mathfrak{S}$ системы $\Xi(h)$ и предполагаем, что $\mathfrak{S}$ - связное множество, содержащее все точки из $\mathfrak{P}$. Подчеркнем, что $P^{j} \neq P^{k}$ при $j \neq k$, но среди отрезков 
$\Sigma^{1}, \ldots, \Sigma^{N}$ могут быть совпадающие (два узла соединены несколькими "параллельными" стержнями). Введем область $\omega^{n} \subset \mathbb{R}^{2}$, ограниченную кусочно гладким контуром $\partial \omega^{n}$, и семейство диффеоморфизмов $\varkappa_{z}^{n}: \mathbb{R}^{2} \rightarrow \mathbb{R}^{2}$, гладко зависящих от параметра $z \in\left[0, l_{n}\right]$. Стержень $G^{n}(h)$ зададим формулой

$$
G^{n}(h)=\left\{x: z^{n} \in\left(0, l_{n}\right), h^{-1} y^{n} \in \omega^{n}\left(z^{n}\right)=\varkappa_{z^{n}}^{n} \omega^{n}\right\}
$$

(ср. определение $(2.50))$. Здесь $\left(y^{n}, z^{n}\right)$ - декартовы координаты, причем $y^{n}=$ $\left(y_{1}^{n}, y_{2}^{n}\right)$ - координаты в плоскости, ортогональной оси $\Sigma^{n}$ стержня $G_{h}^{n}$.

Узел $Q_{h}^{j}=\left\{x \in \mathbb{R}^{3}: h^{-1}\left(x-P^{j}\right) \in Q_{1}^{j}\right\}$ получается сжатием в $h^{-1}$ раз области $Q_{1}^{j}$ относительно центра $P^{j}$. Как обычно, все множества $Q_{1}^{m}, \Sigma^{n}, \omega^{n}$ и т. п. считаем зафиксированными, не зависящими от параметра $h$. Кроме того, $G_{h}^{j} \cap G_{h}^{k}=\varnothing$ при $j \neq k$.

Вывод неравенства Корна для системы стержней $\Xi(h)$ опирается на следующее предположение о соединении стержней и узлов, аналогичное условию $6^{\circ}$ ) из п. 2.6:

$\left.6^{\circ}\right)$ если стержень $G_{h}^{n}$ прикреплен $\kappa$ узлу $Q_{h}^{m}$ (точка $P^{m}$ - конец, отрезка $\left.\Sigma^{n}\right)$, то в пересечении $G_{h}^{n} \cap Q_{h}^{m}$ найдется шар $\mathscr{B}_{h R}^{n, m}$ с радиусом $h R$, причем $R$ не зависит от $h \in\left(0, h_{0}\right]$.

Множество $\Gamma(h)$, по которому сочленение (7.1) защемлено, является объединением частей $\Gamma_{h}^{m_{1}}, \ldots, \Gamma_{h}^{m_{J}}$ поверхностей некоторых узлов $Q_{h}^{m_{1}}, \ldots, Q_{h}^{m_{J}}$, а именно $\Gamma_{h}^{m}=\left\{x: h^{-1}\left(x-P^{m}\right) \in \Gamma^{m}\right\}, \Gamma^{m} \subset \partial Q_{1}^{m}$ и $\operatorname{mes}_{2} \Gamma^{m}>0$. Такое определение влечет за собой выполнение условия $7^{\circ}$ ) из п. 2.6. Рассматриваем случай $J \geqslant 1$, т. е. закреплен хотя бы один узел, и считаем тело (7.1) однородным, т. е. имеем дело с эрзацем (2.6) упругой энергии.

Далее будут указаны нормы нормы | | |... и ||| |||.., обеспечивающие неравенство Корна

$$
\sum_{n=1}^{N}\left|u ; G_{h}^{n}\right|_{\ldots}^{2}+\sum_{m=1}^{M} \mid\left\|u ; Q_{h}^{m}\right\|_{\ldots}^{2} \leqslant c \mathscr{E}(u ; \Xi(h)) .
$$

Строение весовых анизотропных норм в левой части (7.3) определяется после классификации стержней и узлов (защемленные, малоподвижные и подвижнъье), причем категория элемента, предписываемая этой классификацией, обусловлена как его положением в конструкции $\Xi(h)$, так и взаимодействием с другими элементами. Такая классификация, предложенная в [63] (плоский случай) и [39], [25] (пространственный случай), была неизвестна ни в механических, ни в математических исследованиях. С одной стороны, классическое понятие $\mathrm{cma-}$ тически определимой конструкции, широко используемое в теории сопротивления материалов (см. [64], [27] и др.), соотносится лишь с конкретным методом расчета сил и моментов, действующих на элементы, но ни в коем случае не предсказывает зависимость поля смещений $u$ от малого параметра $h$. С другой стороны, в многочисленных работах, посвященных строгому асимптотическому анализу стержневых систем, всем элементам приписываются одинаковые свойства путем жесткой фиксации геометрического строения системы (см., например, [51], [65]-[69] и др.) или постулирования неравенства Корна специального 
вида (см., например, [70], [71]). В статьях [72], [73] В. В. Жикова и С. Е. Пастуховой предложена классификация стержневых систем, упрощенная и неполная в сравнении с изложенной далее, но достаточная для целей теории осреднения. Тем не менее, к конструкциям, содержащим хотя бы один подвижный элемент, перечисленные выше результаты неприменимы. Подчеркнем, что перекладина футбольных ворот (ср. рис. 26 и статью [74]) как раз и оказывается подвижным элементом простейшего сочленения трех стержней.

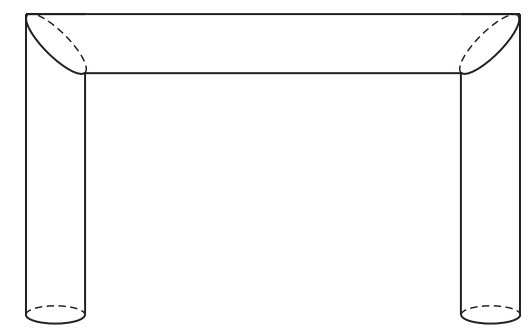

Рис. 26. Футбольные ворота

7.2. Защемленные узлы и стержни. Назовем узел $Q_{h}^{m}$ защемленным, если на части $\Gamma_{h}^{m}$ границы $\partial Q_{h}^{m}$ поставлены условия Дирихле $\left(2.18_{1}\right)$, и поэтому в соответствии с определением (2.17) и предложением 2.1 выполнено соотношение

$$
\left\|u ; Q_{h}^{m}\right\|_{0}^{2}:=\left\|\nabla_{x} u ; L_{2}\left(Q_{h}^{m}\right)\right\|^{2}+h^{-2}\left\|u ; L_{2}\left(Q_{h}^{m}\right)\right\|^{2} \leqslant c \mathscr{E}\left(u ; Q_{h}^{m}\right) .
$$

Стержни, исходящие из защемленного узла, также называются защемленными. Им соответствует весовая анизотропная норма (2.52), в которой $\rho(x)=$ $h+\operatorname{dist}\left\{x, G_{h}^{j} \cap Q_{h}^{m}\right\}-$ весовой множитель, имеющий порядок $O(h)$ на защемленном конце $G_{h}^{j} \cap Q_{h}^{m}$ стержня, и $\left(y_{1}^{j}, y_{2}^{j}, z^{j}\right)$ - декартовы координаты, привязанные к стержню $G_{h}^{j}$. Эту норму теперь обозначаем $\left|u ; G_{h}^{j}\right|_{0}$. В силу теоремы 2.9 выполняется неравенство Корна (2.51).

Обозначим $\mid u ; G_{h}^{j} \mathbf{I}_{\times}$норму (2.51), где $\rho_{h}=1$ (убрали весовые множители). Стержни, с которыми ассоциируется такая норма, называем малоподвижными.

7.3. Малоподвижные узлы. Будем говорить, что узел $Q_{h}^{m}$ малоподвижен в направлении $\tau$, если удалось оценить норму

$$
\begin{aligned}
\left\|u ; Q_{h}^{m}\right\|_{\tau}:= & \left(\int _ { Q _ { h } ^ { m } } \left\{\sum_{i=1}^{2}\left[\left|\frac{\partial u_{i}^{\tau}}{\partial y_{i}^{\tau}}\right|^{2}+h^{1}\left(\left|\frac{\partial u_{i}^{\tau}}{\partial z^{\tau}}\right|^{2}+\left|\frac{\partial u_{3}^{\tau}}{\partial y_{i}^{\tau}}\right|^{2}+\left|u_{i}^{\tau}\right|^{2}\right)\right]\right.\right. \\
& \left.\left.+\left|\frac{\partial u_{3}^{\tau}}{\partial z^{\tau}}\right|^{2}+h^{1}\left(\left|\frac{\partial u_{1}^{\tau}}{\partial y_{2}^{\tau}}\right|^{2}+\left|\frac{\partial u_{2}^{\tau}}{\partial y_{1}^{\tau}}\right|^{2}\right)+h^{-1}\left|u_{3}^{\tau}\right|^{2}\right\} d y^{\tau} d z^{\tau}\right)^{1 / 2}
\end{aligned}
$$

Здесь ось $z^{\tau}$ декартовых координат $\left(y^{\tau}, z^{\tau}\right)$ совпадает с направлением $\tau$, а $u_{i}^{\tau}$ и $u_{3}^{\tau}$ - проекции вектора $u$ на оси $y_{i}^{\tau}$ и $z^{\tau} ; i=1,2$. Рассмотрим узел $Q_{h}^{m}$, присоединенный к малоподвижному (или защемленному) стержню $G_{h}^{j}$, и в качестве $\tau$ возьмем направление $z^{j}$ оси стержня. Следующее утверждение относится как к малоподвижным, так и к защемленным стержням. 
Лемма 7.1. Верна оченка $\left\|u ; Q_{h}^{m}\right\|_{\tau}^{2} \leqslant c\left\{\mathscr{E}\left(u ; Q_{h}^{m}\right)+\mathscr{E}\left(u ; G_{h}^{j}\right)+\mid u ; G_{h}^{j}, \mathbf{l}_{\times}^{2}\right\}$.

Если узел $Q_{h}^{m}$ малоподвижен в любом направлении, ортогональном вектору $\nu$, то он называется подвижсным лищъ в направлении $\nu$. Соответствующая норма выглядит так:

$$
\begin{gathered}
\left\|\left|u ; Q_{h}^{m}\right|\right\|_{[\nu]}:=\left(\int _ { Q _ { h } ^ { m } } \left\{\sum_{i=1}^{2}\left[\left|\frac{\partial u_{i}^{\nu}}{\partial y_{i}^{\nu}}\right|^{2}+h^{1}\left(\left|\frac{\partial u_{i}^{\nu}}{\partial z^{\nu}}\right|^{2}+\left|\frac{\partial u_{3}^{\nu}}{\partial y_{i}^{\nu}}\right|^{2}\right)\right]+h^{1}\left|u_{1}^{\nu}\right|^{2}\right.\right. \\
\left.\left.+h^{-1}\left|u_{2}^{\nu}\right|^{2}+\left|\frac{\partial u_{3}^{\nu}}{\partial z^{\nu}}\right|^{2}+h^{1}\left(\left|\frac{\partial u_{1}^{\nu}}{\partial y_{2}^{\nu}}\right|^{2}+\left|\frac{\partial u_{2}^{\nu}}{\partial y_{1}^{\nu}}\right|^{2}\right)+h^{-1}\left|u_{3}^{\nu}\right|^{2}\right\} d y^{\nu} d z^{\nu}\right)^{1 / 2} .
\end{gathered}
$$

При этом ось $y_{1}^{\nu}$ направлена вдоль вектора $\nu$. Из леммы 7.1 вытекает следующее утверждение.

СлЕДСТвИЕ 7.1. Если узел $Q_{h}^{m}$ присоединен $к$ малоподвижным стержням $G_{h}^{n} u G_{h}^{k}, y$ которых оси лежат в плоскости, перпендикулярной направлению $\nu, и$ не являются параллельными одна другой, то

$$
\left\|\mathbf{u} ; Q_{h}^{m}\right\|_{[\nu]}^{2} \leqslant c\left\{\mathscr{E}\left(u ; Q_{h}^{m}\right)+\mathscr{E}\left(u ; G_{h}^{n}\right)+\mathscr{E}\left(u ; G_{h}^{k}\right)+\left|u ; G_{h}^{n} \mathbf{|}_{\times}^{2}+\right| u ; G_{h}^{k} \mathbf{|}_{\times}^{2}\right\} .
$$

Полностью малоподвижный узел $Q_{h}^{m}$ соотносится с нормой

$$
\begin{aligned}
\left\|u ; Q_{h}^{m}\right\| \|_{\times}:= & \left(\int _ { Q _ { h } ^ { m } } \left\{\sum_{i=1}^{2}\left[\left|\frac{\partial u_{i}}{\partial y_{i}}\right|^{2}+h^{1}\left(\left|\frac{\partial u_{i}}{\partial z}\right|^{2}+\left|\frac{\partial u_{3}}{\partial y_{i}}\right|^{2}\right)+h^{-1}\left|u_{i}\right|^{2}\right]\right.\right. \\
& \left.\left.+\left|\frac{\partial u_{3}}{\partial z}\right|^{2}+h^{1}\left(\left|\frac{\partial u_{1}}{\partial y_{2}}\right|^{2}+\left|\frac{\partial u_{2}}{\partial y_{1}}\right|^{2}\right)+h^{-1}\left|u_{3}\right|^{2}\right\} d y d z\right)^{1 / 2} .
\end{aligned}
$$

В определении (7.7) выбор осей безразличен. Лемма 7.1 обеспечивает очередное утверждение.

СлЕДСТвиЕ 7.2. Если в узле $Q_{h}^{m}$ встречаются три стержня $G_{h}^{n}, G_{h}^{k} u G_{h}^{j}$ с некомпланарными осями, то выполняется неравенство

$$
\begin{aligned}
\left\|\mathbf{u} ; Q_{h}^{m} \mid\right\|_{\times}^{2} \leqslant c\left\{\mathscr{E}\left(u ; Q_{h}^{m}\right)+\mathscr{E}\left(u ; G_{h}^{n}\right)\right. \\
+
\end{aligned}
$$

7.4. Малоподвижные стержни. Очередное утверждение относится к узлу $Q_{h}^{m}$, малоподвижному в направлении $\tau$, которое совпадает с осью стержня $G_{h}^{j}$. Полностью малоподвижный узел и узел, подвижный лишь в направлении $\nu$, которое перпендикулярно $\tau$, обладают нужным свойством, поскольку согласно формулам (7.5)-(7.7) выполнена цепочка неравенств

$$
\left\|u ; Q_{h}^{m}\right\|_{\tau} \leqslant\left\|u ; Q_{h}^{m}\right\|_{[\nu]} \leqslant\left\|u ; Q_{h}^{m}\right\|_{\times} .
$$

Лемма 7.2. Справедливо соотношение $\left|u ; G_{h}^{j}\right|_{\times}^{2} \leqslant c\left\{\mathscr{E}\left(u ; G_{h}^{j}\right)+\left\|\left|u ; Q_{h}^{m}\right|\right\|_{\tau}^{2}\right\}$. 
7.5. Подвижные узлы и балки. Для подвижных стержней и узлов назначим такие нормы:

$$
\begin{aligned}
|\boldsymbol{u} ; G(h)|:= & \left(\int _ { G ( h ) } \left\{\sum_{i=1}^{2}\left[\left|\frac{\partial u_{i}}{\partial y_{i}}\right|^{2}+h^{2}\left(\left|\frac{\partial u_{i}}{\partial z}\right|^{2}+\left|\frac{\partial u_{3}}{\partial y_{i}}\right|^{2}\right)+h^{2}\left|u_{i}\right|^{2}\right]\right.\right. \\
& \left.\left.+h^{2}\left[\left|\frac{\partial u_{1}}{\partial y_{2}}\right|^{2}+\left|\frac{\partial u_{2}}{\partial y_{1}}\right|^{2}+\left|u_{3}\right|^{2}\right]^{2}+\left|\frac{\partial u_{3}}{\partial z}\right|^{2}\right\} d y d z\right)^{1 / 2}, \\
\left\|u ; Q_{h}\right\| \mid:= & \left(\int _ { Q _ { h } } \left\{\sum_{i=1}^{2}\left[\left|\frac{\partial u_{i}}{\partial y_{i}}\right|^{2}+h^{1}\left(\left|\frac{\partial u_{i}}{\partial z}\right|^{2}+\left|\frac{\partial u_{3}}{\partial y_{i}}\right|^{2}+\left|u_{i}\right|^{2}\right)\right]\right.\right. \\
& \left.\left.+\left|\frac{\partial u_{3}}{\partial z}\right|^{2}+h^{1}\left(\left|\frac{\partial u_{1}}{\partial y_{2}}\right|^{2}+\left|\frac{\partial u_{2}}{\partial y_{1}}\right|^{2}+\left|u_{3}\right|^{2}\right)\right\} d y d z\right)^{1 / 2} .
\end{aligned}
$$

Следующая лемма имеет дело либо со стержнем $G_{h}^{j}$, прикрепленным к подвижному узлу $Q_{h}^{m}$, либо с узлом $Q_{h}^{m}$, в который упирается подвижный стержень $G_{h}^{j}$.

Лемма 7.3. Выполняются неравенства

$$
\begin{aligned}
\left|u ; G_{h}^{j}\right|^{2} & \leqslant c\left\{\mathscr{E}\left(u ; G_{h}^{j}+\mathscr{E}\left(u ; Q_{h}^{m}\right)+\left\|u ; Q_{h}^{m}\right\|^{2}\right\},\right. \\
\left\|u ; Q_{h}^{m}\right\|^{2} & \leqslant c\left\{\mathscr{E}\left(u ; Q_{h}^{m}\right)+\mathscr{E}\left(u ; G_{h}^{j}\right)+\left|u ; G_{h}^{j}\right|^{2}\right\} .
\end{aligned}
$$

Доказательства лемм 7.1 и 7.3 изложены подробно в [39] и конспективно в [25]. В [63] аналогичные оценки установлены для сочленений плоских балок (искривленных полосок).

7.6. Неравенство Корна для системы стержней. Утверждения, приведенные в предыдущих пунктах, позволяют переносить "индивидуальные" неравенства Корна с одного элемента конструкции на другой и присваивать этим элементам категории. Для стержней шкала категорий по нисходящей такова:

$$
\text { защемленные \ малоподвижные \ подвижные. }
$$

Она вполне согласуется с неравенствами $\left|u ; G(h) \mathbf{|}_{0} \geqslant\right| \mathbf{|} u ; G(h) \mathbf{|}_{\times} \geqslant \mathbf{|} u ; G(h) \mathbf{|}$, вытекающими непосредственно из определения норм.

Для узлов список (7.9) расширяется до пяти наименований: третью и четвертую позицию занимают соответственно "подвижные лишь в направлении $\nu$ " и "малоподвижные в направлении $\tau$ ", причем из определений (7.4)-(7.7) и $\left(7.8_{2}\right)$ вытекает цепочка неравенств

$$
\|u ; G(h)\|_{0} \geqslant\left|\left\|u ; G(h)\left|\left\|_{\times} \geqslant \mid\right\| u ; G(h)\left\|_{[\nu]} \geqslant\right\|\|u ; G(h)\|\left\|_{\tau} \geqslant\right\| u ; G(h) \| .\right.\right.\right.
$$

Здесь направления $\tau$ и $\nu$ следует считать взаимно перпендикулярными. При нарушении перпендикулярности третье неравенство в (7.10) перестает быть верным, т. е. в отличие от (7.9) классификация узлов не устанавливает отношение порядка. 


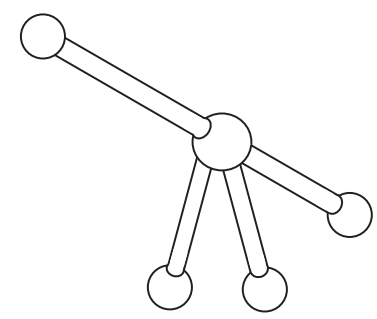

Рис. 27. Колодезный журавль

Приведем пример сочленения, которое содержит элементы всех возможных категорий.

ПримеР 7.1. Колодезный журавль. Приспособление на рис. 27 состоит из пяти узлов и четырех стержней. В соответствии с интерпретацией сочленения пару стержней назовем "ногами", а два оставшихся и продолжающих один другой - "хвостом" и "шеей".

Если закреплена "лапа" одной из ног, то эта нога, разумеется, оказывается защемленной, но все остальные стержни - подвижными. "Туловище" (узел, в котором встречаются четыре стержня) является малоподвижным в одном направлении, а три узла, которые еще не классифицированы, - подвижными полностью.

Если защемлен хвост, то ноги и лапы - подвижные стержни и узлы. Шея малоподвижный стержень, а туловище и "голова" - узлы, малоподвижные в одном направлении. В соответствии с известной поговоркой, заимствованной из русской народной сказки, в последних высказываниях роль хвоста может быть передана шее и наоборот.

Пусть теперь закреплены обе лапы журавля. Теперь туловище - узел, подвижный лишь в одном направлении. Шея и хвост, а также узлы на их концах полностью подвижные элементы конструкции.

Наконец, в случае защемления обеих ног и хвоста становятся малоподвижными шея и туловище, но голова - малоподвижной только в одном направлении.

Классификация стержней и узлов производится итерированием излагаемой ниже процедуры. Сначала всем элементам присваивается низшая категория подвижные. Учитывая сформулированные в пп. 7.2-7.5 утверждения, переберем все пути в скелете $\mathfrak{S}$, начинающиеся в защемленных узлах и не возвращающиеся на пройденные ими стержни. Таких путей конечное число и до любого элемента можно дотянуться одним из них, так как скелет $\mathfrak{S}$ связен. Первая попытка выяснить категории элементов обычно не приводит к успеху (исключение: $\mathfrak{S}$ - ломаная), так как имеются узлы, лежащие на пресечении нескольких путей. Для таких узлов среди категорий, порожденных разными путями, либо выбирается самая высокая, либо при помощи следствий 7.1 и 7.2 категории объединяются в более высокую, а именно $[\nu]$ или $\times$. Если произошло изменение категории хотя бы одного узла, то могут улучшиться и категории 
стержней. Поэтому прохождение всевозможных путей следует повторить. Поскольку категории обязательно повышаются, количество ${ }^{9}$ повторов не превосходит $4 M+2 N$.

Предложенный алгорифм не претендует на оптимальность, и, конечно же, он может быть усовершенствован. Для конкретных конструкций предпочтительной оказывается процедура поиска, напоминающая детскую игру "puzzle" и оперирующую со стандартными группами “узлы-стержни”. Проведенная классификация элементов конструкции совместно с оценками, установленными в пп. 7.2-7.5, обеспечивают следующее утверждение.

Теорема 7.1. На сочленении $\Xi(h)$ справедливо весовое неравенство Корна (7.3), где $\left|u ; G_{h}^{n}\left\|_{\ldots}^{2} u \mid\right\| u ; Q_{h}^{m} \|_{\ldots}^{2}\right.$ - нормы, отвечающие установленным категориям стержня $G_{h}^{n}$ и узла $Q_{h}^{m}$.

Согласно формулам (7.8), выполняются оценки $\left\|u ; G_{h}^{n} \mathbf{l} \geqslant c h\right\| u ; H^{1}\left(G_{h}^{n}\right) \|$ и \|\|$u ; Q_{h}^{m}\left\|\mid \geqslant c h^{1 / 2}\right\| u ; H^{1}\left(G_{h}^{n}\right) \|$ с положительной постоянной $c$. Таким образом, при игнорировании категорий элементов теорема 7.1 обеспечивает упрощенный вариант неравенства Корна.

СЛЕДСТВИЕ 7.3. Множитель $c(h)$ в неравенстве

$$
\left\|u ; H^{1}(\Xi(h))\right\|^{2} \leqslant c(h) \mathscr{E}(u ; \Xi(h))
$$

для поля $и \in \stackrel{\circ}{H^{1}}(\Xi(h), \Gamma(h))^{3}$ удовлетворяет соотношению $c(h) \leqslant C h^{2}$.

Подчеркнем, что при использовании обычной, а не весовой анизотропной нормы в пространстве $H^{1}(\Xi(h))$ приведенная оценка для постоянной $c(h)$ в неравенстве (7.3) оказывается асимптотически точной (см. работы [69], [63], [72]).

7.7. Обсуждение. Как и в примере 2.5 , для того чтобы убедиться в асимптотической точности неравенства Корна, достаточно рассмотреть лишь начальные члены асимптотического анзаца $(2.53)$, в котором $w^{n}=\left(w_{1}^{n}, \ldots, w_{4}^{n}\right)$ - произвольная гладкая вектор-функция.

Если стержень $G_{h}^{n}$ закреплен за конец $z^{n}=0$, вектор-функцию $w^{n}$ следует подчинить граничным условиям

$$
w^{n}(0)=0 \in \mathbb{R}^{4}, \quad \partial_{z} w_{1}^{n}(0)=\partial_{z} w_{2}^{n}(0)=0 .
$$

Таким образом, слагаемые в норме $\mid u ; G_{h}^{n} \mathbf{|}_{0}$ приобретают порядок $1=h^{0}$, а рост весовых множителей $\rho_{h}$ компенсируется вырождением компонент $w_{n}$ согласно условиям Дирихле (7.11). Как обычно, исключение составляют производные $\partial u_{i}^{n} / \partial y_{i}, i=1,2$, которые аннулируются для компонент $\left(2.53_{1}\right)$ и $\left(2.53_{2}\right)$, но присутствуют как в функционале энергии (2.11), так и во всех выражениях $\mathbf{|} u ; G_{h}^{n} \mathbf{|}_{\ldots}^{2}$ (ср. пример 2.5).

Если стержень $G_{h}^{n}$ малоподвижен, то какие-либо граничные условия для $w^{n}$ не назначаются. Это согласуется с отсутствием весовых множителей $\rho_{h}$ в норме $\mid u ; G_{h}^{n} \mathbf{I}_{\times}$, слагаемые которой по-прежнему суть $O\left(h^{2}\right)$ при $h \rightarrow+0$.

В случае подвижного стержня $G_{h}^{n}$ в норме $\left(7.8_{1}\right)$ появляется еще одно исключительное слагаемое $\int h^{2}\left|u_{3}^{j}\right|^{2} d y d z=O\left(h^{4}\right)$ (интегрирование ведется по $\left.G_{h}^{n}\right)$.

\footnotetext{
${ }^{9} \mathrm{~B}$ статьях [39], [25] ошибочно указано число $5 N+3 M$.
} 
Для придания ему порядка $h^{2}$ изменим анзац (2.53), добавив в него жесткое продольное смещение стержня, а именно,

$$
u_{3}^{n}(h, x)=h^{-1} a^{n}+w_{3}^{n}(z)-h^{-1}\left(y_{1} \partial_{z} w_{1}^{n}(z)+y_{2} \partial_{z} w_{2}^{n}(z)\right) .
$$

Множитель $a^{n}$ берем постоянным, поскольку при переменной величине $a^{n}(z)$ интеграл $\int\left|\partial_{z} u_{3}^{n}(y, z)\right|^{2} d y d z$ приобретает недопустимо большой порядок $O\left(h^{0}\right)$. Отметим, что новое слагаемое не влияет на функционал энергии $\mathscr{E}\left(u^{n} ; \Xi(h)\right)$, содержащий лишь производные смещений. Асимптотический анализ [74] простейшей двумерной задачи (см. рис. 23) показывает, что продольное жесткое смещение подвижного стержня действительно сравнимо с его изгибами, т. е. множители при $a_{0}^{n} e^{3}$ и $w_{i}^{n}(z) e^{i}$ должны быть одинаковыми. Это обстоятельство имеет серьезные последствия: одномерная модель деформации системы стержней с подвижными элементами перестает быть дифференциальной задачей за счет появления дополнительных алгебраических неизвестных $a_{3}^{n}$, возникновения нелокальных условий сопряжения и необходимости проецирования некоторых уравнений на пространство функций с нулевым средним (подробности см. в статьях [39], [25]).

Итак, асимптотические анзацы (2.53) и (7.12) указывают на правильность распределения весовых множителей в нормах $\left|\cdot ; G_{h}^{n}\right| \ldots$ для защемленных, малоподвижных и подвижных стержней. Структура асимптотических анзацев для узлов более простая: в качестве главного приближения на $Q_{n}^{m}$ выступает жесткое смещение

$$
u^{m}(h, x)=d\left(x-P^{m}\right)\left[h^{-1} \mathbf{a}^{m}+h^{0} \mathbf{b}^{m}\right]
$$

здесь $d(x)$ - матрица (2.8), а $\mathbf{a}^{m} \in \mathbb{R}^{6}$ и $\mathbf{b}^{m} \in \mathbb{R}^{3} \times \mathbb{O}^{3}$ - столбцы (три нижних компоненты столбца $\mathbf{b}^{m}$ равны нулю). Устойчивые краевые условия и устойчивые условия сопряжения получаются в результате согласования анзацев (2.53), (7.12) и (7.13). Например,

$$
\mathbf{a}^{m}=0, \quad \mathbf{b}^{m}=0
$$

для защемленного узла, а значит, условия Дирихле (7.11) равносильны совпадению выражений (7.13) и (2.53) на узле $Q_{h}^{m}$ с точностью $O\left(h^{1}\right)$. Вспоминая, что анзацы $(2.53)$ и (7.13) соответствуют энергетическому функционалу $\mathscr{E}(u ; \Xi(h))=O\left(h^{2}\right)$, видим, что формулы (7.14) вытекают также из соотношения $|u ; G(h)|_{0} \leqslant c h^{2}$, обеспеченного неравенством Корна (7.3). Вычисляя нормы для остальных категорий узлов, заключаем, что

$$
\begin{aligned}
& \mathbf{a}_{1}^{m}=\mathbf{a}_{2}^{m}=\mathbf{a}_{3}^{m}=0 \quad \text { (полностью малоподвижный узел), } \\
& \mathbf{a}_{1}^{m}=\mathbf{a}_{2}^{m}=0 \quad \text { (узел, подвижный лишь в направлении } \nu \text { ), } \\
& \mathbf{a}_{3}^{m}=0 \quad \text { (узел, малоподвижный в направлении } \tau=e^{3} \text { ). }
\end{aligned}
$$

Наконец, для подвижного узла новые ограничения на столбцы $\mathbf{a}^{m}$ и $\mathbf{b}^{m}$ не возникают. Полученная дополнительная информация о столбцах $\mathbf{a}^{m}$ и $\mathbf{a}^{m}$ в (7.13) позволяет корректно поставить условия сопряжения в узлах для одномерной 
модели деформации системы стержней. Постановка этих условий описана в [39] (двумерная ситуация) и [25] (трехмерная ситуация).

Насколько известно автору, указанные выше две статьи - единственные, где производился асимптотический анализ произвольных конечных систем стержней; например, постулируемое в [70], [71] специальное неравенство типа Пуанкаре-Корна не может быть выполнено для конструкций, содержащих подвижнъце элементы. Упомянем публикации [51], [66], [68]-[71], [75]-[77], посвященные задачам осреднения периодических систем тонких стержней, количество элементов в которых неограниченно возрастает при уменьшении размеров ячейки периодичности. Доказательства неравенств Корна для периодических стержневых конструкций, приведенные в [69] и [72], [73], неявно используют приемы, разработанные соответственно в статьях [8] (см. теорему 2.5) и [63] (ср. двумерный аналог следствия 7.2).

\section{Список литературы}

[1] A. Korn, "Solution générale du problème d'équilibre dans la théorie de l'élasticité, dans le cas où les efforts sont donnés à la surface", Ann. Fac. Sci. Toulouse Math. (2), 10 (1908), 165-269.

[2] K. O. Friedrichs, "On the boundary-value problems of the theory of elasticity and Korn's inequality", Ann. of Math. (2), 48:2 (1947), 441-471.

[3] J. Gobert, "Une inégalité fondamentale de la théorie de l'élasticité", Bull. Soc. Roy. Sci. de Liège, 31 (1962), 182-191.

[4] J. Nečas, Les méthodes directes en théorie des équations elliptiques, Masson, Paris; Academia, Prague, 1967.

[5] П. П. Мосолов, В. П. Мясников, "Доказательство неравенства Корна", Докл. АН CCCP, 201 (1971), 36-39; англ. пер.: P. P. Mosolov, V.P. Myasnikov, "A proof of Korn's inequality", Soviet Math. Dokl., 12 (1971), 1618-1622.

[6] Г. Дюво, Ж.-Л. Лионс, Неравенства в механике и физике, Наука, М., 1980; пер. с франц.: G. Duvaut, J.-L. Lions, Les inéquations en mécanique et en physique, Travaux et Recherches Mathématiques, 21, Dunod, Paris, 1972.

[7] В. А. Кондратьев, О. А. Олейник, "Краевые задачи для системы теории упругости в неограниченных областях. Неравенство Корна", УМН, 43:5 (1988), 55-98; англ. пер.: V.A. Kondrat'ev, O.A. Oleinik, "Boundary-value problems for the system of elasticity theory in unbounded domains. Korn's inequalities", Russian Math. Surveys, 43:5 (1988), 65-119.

[8] Б.А. Шойхет, "Об асимптотически точных уравнениях тонких плит сложной структуры", ПММ, 37:5 (1973), 914-924; англ. пер.: В. А. Shoikhet, "On asymptotically exact equations of thin plates of complex structure", J. Appl. Math. Mech., 37:5 (1973), 867-877.

[9] С. А. Назаров, "Неравенства Корна, асимптотически точные для тонких областей", Вестник СПбГУ. Сер. матем., мех., астроном., 2 (1992), 19-24; англ. пер.: S. A. Nazarov, "Korn inequalities that are asymptotically exact for thin domains", Vestnik St. Petersburg Univ. Math., 2 (1992), 18-22.

[10] С. А. Назаров, "Обоснование асимптотической теории тонких стержней. Интегральные и поточечные оценки”, Проблемы математического анализа, Вып. 17, СПбГУ, СПб, 1997, 101-152.

[11] D. Cioranescu, O. A. Oleinik, G. Tronel, "Korn's inequalities for frame type structures and junctions with sharp estimates for the constants", Asymptot. Anal., 8:1 (1994), $1-14$. 
[12] С.А. Назаров, “Асимптотический анализ произвольно анизотропной пластины переменной толщины (пологой оболочки)", Матем. сб., 191:7 (2000), 129-159; англ. пер.: S. A. Nazarov, "Asymptotic analysis of an arbitrary anisotropic plate of variable thickness (sloping shell)", Sb. Math., 191:7 (2000), 1075-1106.

[13] С.А. Назаров, А.С. Слуцкий, "Одномерные уравнения деформации тонких слабоискривленных стержней. Асимптотический анализ и обоснование", Изв. PAH. Сер. матем., 64:3 (2000), 97-130; англ. пер.: S. A. Nazarov, A. S. Slutskii, "One-dimensional equations of the deformation of thin slightly curved rods. Asymptotic analysis and justification", Izv. Math., 64:3 (2000), 531-562.

[14] Е.А. Акимова, С.А. Назаров, Г.А. Чечкин, "Асимптотика решения задачи о деформации произвольной локально периодической тонкой пластины", $T p$. MMO, 65 (2004), 3-34; англ. пер.: E. A. Akimova, S. A. Nazarov, G. A. Chechkin, "Asymptotics of the solution of the problem of the deformation of an arbitrary locally periodic thin plate", Trans. Moscow Math. Soc., 2004, 1-29.

[15] С. А. Назаров, Асимптотическая теория тонких пластин и стержней. Понижение размерности и интегралъные оченки, Научная книга, Новосибирск, 2002.

[16] С. А. Назаров, "Весовые неравенства Корна на параболоидальных областях", Maтем. заметки, 62:5 (1997), 751-765; англ. пер.: S. A. Nazarov, "Weighted Korn inequalities in paraboloidal domains", Math. Notes, 62:5 (1997), 629-641; C. A. Haзаров, "Письмо в редакцию", Матем. заметки, 63:4 (1998), 640; англ. пер.: S. A. Nazarov, "Letter to the editor", Math. Notes, 63:4 (1998), 565.

[17] С. А. Назаров, А.С. Слуцкий, "Принцип Сен-Венана для параболоидальных упругих тел", Проблемы математического анализа, Вып. 18, СПбГУ, СПб, 1999, $133-180$.

[18] O. A. Oleinik, G. A. Yosifian, "On the asymptotic behavior at infinity of solutions in linear elasticity", Arch. Ration. Mech. Anal., 78:1 (1982), 29-53.

[19] D. Cioranescu, O. A. Oleinik, G. Tronel, "On Korn's inequalities for frame type structures and junctions", C. R. Acad. Sci. Paris Sér. I Math., 309:9 (1989), 591-596.

[20] V.A. Kozlov, V. G. Maz'ya, A. B. Movchan, "Asymptotic representation of elastic fields in a multi-structure", Asymptot. Anal., 11:4 (1995), 343-415.

[21] V.A. Kozlov, V.G. Maz'ya, A.B. Movchan, Asymptotic analysis of fields in multi-structures, Oxford Math. Monogr., Clarendon Press, Oxford, 1999.

[22] S. A. Nazarov, "Korn's inequalities for junctions of spatial bodies and thin rods", Math. Methods Appl. Sci., 20:3 (1997), 219-243.

[23] С. А. Назаров, "Неравенство Корна для упругого соединения тела со стержнем", Проблемь механики деформируемого твердого тела, СПбГУ, СПб, 2002, 234-240.

[24] С. А. Назаров, "Асимптотический анализ и моделирование сочленения массивного тела с тонкими стержнями”, Тр. сем. им. И.Г. Петровского, Вып. 24, МГУ, M., 2004, 95-214; англ. пер.: "Asymptotic analysis and modeling of the junction of a massive body and thin rods", J. Math. Sci., 127:5 (2003), 2172-2263.

[25] С. А. Назаров, А. С. Слуцкий, "Асимптотический анализ произвольной пространственной системы тонких стержней”, Тр. С.-Петербург. матем. о-ва, 10 (2004), 63-115; англ. пер.: "Asymptotic analysis of an arbitrary spatial system of thin rods", Proceedings of the St. Petersburg Mathematical Society. Vol. X, Amer. Math. Soc. Ser. 2, 214, Amer. Math. Soc., Providence, R.I., 2005.

[26] С. Г. Лехницкий, Теория упругости анизотропного тела, Наука, М., 1977; англ. пер.: S. G. Lekhnitskij, Theory of elasticity of an anisotropic body, Mir Publishers, Moscow, 1981.

[27] Ю.Н. Работнов, Механика деформируемого твердого тела, Учебное пособие, Наука, М., 1988.

[28] М.Ш. Бирман, М. З. Соломяк, Спектральная теория самосопряженных операторов в гилъбертовом пространстве, изд-во Ленингр. ун-та, Л., 1980; англ. пер.: M. Sh. Birman, M. Z. Solomjak, Spectral theory of selfadjoint operators in Hilbert space, Reidel, Dordrecht, 1987. 
[29] О. А. Олейник, Г. А. Иосифиан, А. С. Шамаев, Математические задачи теории сильно неоднородных упругих сред, МГУ, М., 1990.

[30] B. Bernstein, R. A. Toupin, "Korn inequality for the sphere and circle", Arch. Ration. Mech. Anal., 6 (1960), 51-64.

[31] L. E. Payne, H. F. Weinberger, "On Korn's inequality", Arch. Ration. Mech. Anal., 8 (1961), 89-98.

[32] C. O. Horgan, "Korn's inequalities and their application in continuum mechanics", SIAM Rev., 37:4 (1995), 491-511.

[33] В.Г. Мазья, Пространства С.Л. Соболева, ЛГУ, Л., 1985; англ. пер.: V. G. Maz'ya, Sobolev spaces, Springer Ser. Soviet Math., Springer, Berlin, 1985.

[34] Г. Фикера, Теоремы существования в теории упругости, Мир, М., 1974; пер. с англ.: G. Fichera, Existence theorems in elasticity, Springer-Verlag, Berlin, 1972.

[35] И. Главачек, Я. Гаслингер, И. Нечас, Я. Ловишек, Решение вариачионных неравенств в механике, Мир, М., 1986; англ. пер.: I. Hlavaček, J. Haslinger, J. Nečas, J. Lovišek, "Solution of variational inequalities in mechanics", Appl. Math. Sci., 66, Springer-Verlag, New York, 1988.

[36] O. A. Oleinik, "Korn's type inequalities and application to elasticity", International conference in memory of Vito Volterra (Rome, 1990), Atti Convegni Lincei, 92, 1992, 183-209.

[37] P. G. Ciarlet, Mathematical elasticity, II: Theory of plates, Studies in Mathematics and its Applications, 27, North-Holland, Amsterdam, 1997.

[38] С. А. Назаров, “Оценки вторых производных собственных векторов для тонких анизотропных пластин с переменой толщиной”, Записки науч. сем. ПОМИ, 308 (2004), 161-181; англ. пер.: S. A. Nazarov, "Estimates for the second derivatives of eigenvectors for thin anisotropic plates with variable thickness", J. Math. Sci., 132:1 (2006), 91-102.

[39] С. А. Назаров, А. С. Слуцкий, "Неравенство Корна для произвольной системы тонких искривленных стержней", Сиб. матем. журн., 43:6 (2002), 1319-1331; англ. пер.: S. A. Nazarov, A. S. Slutskii, "Korn's inequality for an arbitrary system of distorted thin rods", Siberian Math. J., 43:6 (2002), 1069-1073.

[40] С. А. Назаров, "Весовое анизотропное неравенство Корна для сочленения пластины со стержнями", Матем. сб., 195:4 (2004), 97-126; англ. пер.: S. A. Nazarov, "Weighted anisotropic Korn's inequality for a junction of a plate and a rod", $S b$. Math., 195:4 (2004), 553-583.

[41] Е.А. Акимова, С.А. Назаров, Г.А. Чечкин, "Весовое неравенство Корна: процедура "тетрис", обслуживающая произвольную периодическую пластину", Докл. РАН, 380:4 (2001), 439-442; англ. пер.: Е. A. Akimova, S. A. Nazarov, G. A. Chechkin, "The weighted Korn inequality: The"tetris" procedure that serves for an arbitrary periodic plate", Dokl. Math., 64:2 (2001), 205-207.

[42] В.Г. Мазья, С.А. Назаров, "Парадоксы предельного перехода в решениях краевых задач при аппроксимации гладких областей многоугольными", Изв. АН СССР. Сер. матем., 50:6 (1986), 1156-1177; англ. пер.: V. G. Maz'ya, S. A. Nazarov, "Paradoxes of limit passage in solutions of boundary value problems involving the approximation of smooth domains by polygonal domains", Math. USSR-Izv., 29:3 (1987), 511-533.

[43] V. A. Kozlov, V. G. Maz'ya, A. B. Movchan, "Fields in non-degenerate 1D-3D elastic multi-structures", Quart. J. Mech. Appl. Math., 54:2 (2001), 177-212.

[44] S. A. Nazarov, "Junctions problems of bee-on-ceiling type in the theory of anisotropic elasticity", C. R. Acad. Sci. Paris Sér. I Math., 320:11 (1995), 1419-1424.

[45] С. А. Назаров, “Асимптотика решений задачи теории упругости для трехмерного тела с тонкими отростками", Докл. РАН, 352:4 (1997), 458-461; англ. пер.: S. A. Nazarov, "Asymptotics of solutions to the elasticity-theoretic problem for a three-dimensional body with thin branches", Dokl. Math., 55:1 (1997), 87-90. 
[46] С. А. Назаров, “Эллиптические задачи на гибридных областях", Функи. анализ и его прил., 38:4 (2004), 55-72; англ. пер.: S. A. Nazarov, "Elliptic boundary value problems in hybrid domains", Funct. Anal. Appl., 38:4 (2004), 283-297.

[47] И.И. Аргатов, С. А. Назаров, "О равновесии упругого тела на пронизывающих его горизонтальных тонких упругих стержнях", Прикл. мех. и техн. физ., 40:4 (1999), 236-242; англ. пер.: I. I. Argatov, S. A. Nazarov, "Equilibrium of an elastic body pierced by horizontal thin elastic bars", J. Appl. Mech. Tech. Phys., 40:4 (1999), 763-769.

[48] И.И. Аргатов, С. А. Назаров, "Асимптотический анализ на соединениях областей различных предельных размерностей. Упругое тело, пронзенное тонкими стержнями", Проблемы математического анализа, Вып. 20, 2000, 3-55; англ. пер.: I. I. Argatov, S. A. Nazarov, "Asymptotic analysis of problems in junctions of domains of different limit dimension. An elastic body pierced by thin rods", J. Math. Sci., 102:5 (2000), 4349-4387.

[49] Г. П. Панасенко, "Осреднение полей в композиционных материалах с высокомодульной арматурой", Вестн. МГУ. Сер. ХV. Вычисл. матем. и кибернет., 1983, № 2, 20-27; англ. пер.: G.P. Panasenko, "Averaging fields in composite materials with high-elastic modulus reinforcement", Moscow Univ. Comput. Math. Cybernet., 2 (1983), 23-31.

[50] Г. П. Панасенко, "Многокомпонентное осреднение процессов в сильно неоднородных структурах", Матем. сб., 181:1 (1990), 134-142; англ. пер.: G. P. Panasenko, "Multicomponent homogenization for processes in essentially nonhomogeneous structures", Math. USSR-Sb., 69:1 (1991), 143-153.

[51] Н.С. Бахвалов, Г. П. Панасенко, Осреднение процессов в периодических средах. Математические задачи механики композиционных материалов, Наука, M., 1984; англ. пер.: N. S. Bakhvalov, G. P. Panasenko, Homogenisation: averaging processes in periodic media. Mathematical problems in the mechanics of composite materials, Math. Appl. (Soviet Ser.), 36, Kluwer, Dordrecht, 1989.

[52] С. А. Назаров, "Неравенства Корна для сочленений упругих тел с тонкими пластинами", Сиб. матем. журн., 46:4 (2005), 876-889; англ. пер.: S. A. Nazarov, "Korn's inequalities for junctions of elastic bodies with thin plates", Siberian Math. J., 46:4 (2005), 695-706.

[53] D. Caillerie, "The effect of a thin inclusion of high rigidity in an elastic body", Math. Methods Appl. Sci., 2:3 (1980), 251-270.

[54] P. G. Ciarlet, H. Le Dret, R. Nzengwa, "Modélisation de la jonction entre un corps élastique tridimensionnel et une plaque", C. R. Acad. Sci. Paris. Sér. I Math., 305:2 (1987), 55-58.

[55] M. Aufranc, "Numerical study of a junction between a three-dimensional elastic structure and a plate", Comput. Methods Appl. Mech. Engrg., 74:2 (1989), 207-222.

[56] D. Leguillon, E. Sanchez-Palencia, "Approximation of a two-dimensional problem of junctions", Comput. Mech., 6:5-6 (1990), 435-455.

[57] P. G. Ciarlet, Plates and junctions in elastic multi-structures. An asymptotic analysis, Rech. Math. Appliquées, 14, Masson, Paris; Springer, Berlin, 1990.

[58] Физики продолжают шутить, Мир, М., 1968.

[59] A. Gaudiello, R. Monneau, J. Mossino, F. Murat, A. Sili, "On the junction of elastic plates and beams", C. R. Acad. Sci. Paris. Sér. I Math., 335:8 (2002), 717-722.

[60] O. V. Izotova, S.A. Nazarov, G.H. Sweers, "Weighted Korn inequalities for thin-walled elastic structures", C. R. Mecanique, 334:12 (2006), 707-712.

[61] О.В.Изотова, С. А. Назаров, Г. Х. Свирс, "Асимптотически точное весовое неравенство Корна для тонкостенных упругих конструкций", Проблемы математического анализа, Вып. 36, Научная книга, Новосибирск, 2007.

[62] Дж. Хокинс, Дж. Уайт, Разгадка тайны Стоунхенджа, Мир, М., 1973; пер. с англ.: G. S. Hawkins, J. B. White, Stonehenge Decoded, Doubleday, Garden City, 1965. 
[63] С. А. Назаров, А.С. Слуцкий, "Произвольные плоские системы анизотропных балок", Тр. МИАН, 236, Наука, М., 2002, 234-261; англ. пер.: S. A. Nazarov, A.S. Slutskij, "Arbitrary plane systems of anisotropic beams", Proc. Steklov Inst. Math., 236:1 (2002), 222-249.

[64] Н. М. Беляев, Сопротивление материалов, Наука, М., 1965.

[65] H. Le Dret, "Modeling of the junction between two rods", J. Math. Pures Appl. (9), 68:3 (1989), 365-397.

[66] D. Cioranescu, J. Saint Jean Paulin, "Structures très minces en élasticité linéarisée: tours et grillages", C. R. Acad. Sci. Paris. Sér. I Math., 308:2 (1989), 41-46.

[67] H. Le Dret, Problèmes variationnels dans les multi-domains. Modélisation des jonctions et applications, Rech. Math. Appl., 19, Masson, Paris, 1991.

[68] D. Cioranescu, J. Saint Jean Paulin, Homogenization of reticulated structures, Appl. Math. Sci., 136, Springer-Verlag, New York, 1999.

[69] В.В. Жиков, "Усреднение задач теории упругости на сингулярных структуpax", Изв. РАН. Сер. матем., 66:2 (2002), 81-148; англ. пер.: V. V. Zhikov, "Homogenization of elasticity problems on singular structures", Izv. Math., 66:2 (2002), 299-365.

[70] Г.П. Панасенко, "Асимптотические решения системы теории упругости для стержневых и каркасных структур", Матем. сб., 183:1 (1992), 89-113; англ. пер.: G.P. Panasenko, "Asymptotic solutions of a system in the theory of elasticity for rod and frame structures", Sb. Math., 75:1 (1993), 85-110.

[71] G. P. Panasenko, "Asymptotic analysis of bar systems. I", Russian J. Math. Phys., 2:3 (1994), 325-352; "Asymptotic analysis of bar systems. II", Russian J. Math. Phys., 4:1 (1996), 87-116.

[72] В. В. Жиков, С. Е. Пастухова, "О неравенствах Корна на тонких периодических каркасах", Современная математика и ее приложения, 2 (2003), 25-45; англ. пер.: V. V. Zhikov, S. E. Pastukhova, "On Korn inequalities on thin periodic frames", J. Math. Sci., 123:5 (2004), 4499-4521.

[73] В. В. Жиков, С.Е. Пастухова, "Вывод предельных уравнений теории упругости на тонких сетках", Тр. сем. им. И. Г. Петровского, 25, 2005, 55-97; англ. пер.: V. V. Zhikov, S. E. Pastukhova, "Derivation of the limit equations of elasticity theory on thin nets", J. Math. Sci., 135:1 (2006), 2637-2665.

[74] С. А. Назаров, А. С. Слуцкий, “Асимптотика частот собственных колебаний упругих балок, соединенных в форме буквы П”, Докл. РАН, 380:1 (2001), 23-26; англ. пер.: S. A. Nazarov, A.S. Slutskii, "Asymptotics of the frequencies of natural oscillations of elastic beams joined in the form of the letter $\Pi$ ", Dokl. Math., 64:2 (2001), 266-269.

[75] С. Е. Пастухова, "Усреднение задач теории упругости на периодических ящичных структурах критической толщины”, Докл. РАН, 387:4 (2002), 447-451; англ. пер.: S. E. Pastukhova, "Homogenization of elasticity problems on periodic box structures of critical thickness", Dokl. Math., 66:3 (2002), 369-373.

[76] В.В.Жиков, С. Е. Пастухова, "Усреднение задач теории упругости на периодических сетках критической толщины”, Матем. сб., 194:5 (2003), 61-95; англ. пер.: V.V. Zhikov, S.E. Pastukhova, "Homogenization for elasticity problems on periodic networks of critical thickness", Sb. Math., 194:5 (2003), 697-732.

[77] C. Е. Пастухова, "Усреднение задач теории упругости на периодических стержневых каркасах критической толщины”, Докл. РАН, 394:1 (2004), 26-31; англ. пер.: S. E. Pastukhova, "Homogenization of elasticity problems on periodic rod frames of critical thickness", Dokl. Math., 69:1 (2004), 20-25.

\section{C. А. Назаров (S. A. Nazarov)}

Поступила в редакцию

Институт проблем машиноведения РАН 15.10.2007

E-mail: serna@snark.ipme.ru 